\title{
Dimension-8 operators in the Standard Model Effective Field Theory
}

\section{Christopher W. Murphy}

Home Point Financial, Ann Arbor, MI 48105, U.S.A.

E-mail: chrismurphybnl@gmail.com

ABSTRACT: We present a complete basis of dimension- 8 operators in the Standard Model Effective Field Theory. Attention is paid to operators that vanish in the absence of flavor structure. The 44,807 operators are encoded in 1,031 Lagrangian terms. We also briefly discuss a few aspects of phenomenology involving dimension-8 operators, including lightby-light scattering and electroweak precision data.

Keywords: Effective Field Theories, Beyond Standard Model

ARXIV EPRINT: 2005.00059 


\section{Contents}

1 Introduction 1

2 Notation and conventions $\quad 3$

3 Operator classification 5

3.1 Bosonic operators 6

$\begin{array}{lll}3.2 & \text { Two-fermion operators } & 7\end{array}$

$\begin{array}{lll}3.3 & \text { Four-fermion operators } & 10\end{array}$

4 The complete set of dimension-8 operators 16

$\begin{array}{llr}4.1 & \text { Results for bosonic operators } & 18\end{array}$

$\begin{array}{lll}4.2 & \text { Results for two-fermion operators } & 19\end{array}$

$\begin{array}{lll}4.3 & \text { Results for four-fermion operators } & 19\end{array}$

5 Phenomenology 19

$\begin{array}{lll}5.1 & \text { Light-by-light scattering } & 26\end{array}$

$\begin{array}{lll}5.2 & \text { Electroweak precision data } & 35\end{array}$

$\begin{array}{lll}5.3 & \text { Scalar } \mathrm{SU}(2)_{w} \text { quartets } & 37\end{array}$

6 Comments on renormalization group evolution 39

$\begin{array}{lll}7 & \text { Conclusions } & 40\end{array}$

$\begin{array}{ll}\text { A Dimension-6 and -7 operators } & 41\end{array}$

\section{Introduction}

The Standard Model (SM) is an extremely successful theory that has been rigorously tested at the Large Hadron Collider (LHC) and elsewhere. Nevertheless it is widely expected that the SM is only an effective field theory (EFT), valid up to some cutoff scale $\Lambda$. The Standard Model Effective Field Theory (SMEFT) generalizes the SM by adding a complete, but not over-complete basis of operators at every mass-dimension $d$ rather than stopping at $d=4 .^{1}$

The counting and classification of operators in the SMEFT has a long history. Starting with dimension-5 there is a single type operator [1], $N_{\text {type }}=1$, and it violates lepton number. At dimension-6, ref. [2] classified the 76 baryon number preserving (B) Lagrangian

\footnotetext{
${ }^{1}$ The SMEFT assumes there are no light hidden states such as sterile neutrinos or an axion, and that the Higgs boson form part of an $\mathrm{SU}(2)_{w}$ doublet with hypercharge $\mathrm{y}=1 / 2$. Other types of EFTs are possible where these assumptions are relaxed, but we do not consider them here.
} 
terms; see [3] for earlier work. The eight baryon number violating $(\not B)$ terms were previously known [4], yielding a total of $N_{\text {term }}=84$. In terms of actual operators rather than terms in the Lagrangian, the counts explode when flavor structure is allowed. For three generations of fermions, $n_{g}=3$, there are $N_{\text {op }}=2499$ independent $B$ operators [5] and 546 $\not B$ operators [6]. Hilbert series methods were applied to the SMEFT in refs. [7-10], providing an elegant way to count the number of operators for arbitrary dimension $d$. Computing tools Sim2Int [11], DEFT [12], BasisGen [13], ECO [14], and GrIP [15] were subsequently developed, allowing for automated counting of operators.

Beyond counting operators, work has been done on their explicit forms as well. Refs. $[16,17]$ classified the 18 dimension- 7 operators. So far only partial sets of dimension- 8 operators exist in the literature. This includes, however, all of the bosonic operators (in a basis where the number of derivatives is minimized) [18-20]. Our goal in this work is to find a complete set of dimension- 8 operators. A subtlety in constructing the dimension8 operator basis is that some operators vanish in the absence of flavor structure. Our basis contains 231 types of operators corresponding to 1031 Lagrangian terms. For comparison, with $n_{g}=1$ there are 993 operators, while for $n_{g}=3$ there are instead 44807 operators [10]. We find there are 38 terms that vanish when $n_{g}=1$, consistent with the counting of ref. [10].

Although the counting and classification of operators is certainly interesting in its own right, there is also a wide range of phenomenological implications of dimension- 8 operators as well. For some phenomena dimension- 8 is the lowest dimension where the interactions become possible. Most famous among these processes is light-by-light scattering. Another area where dimension- 8 effects have been studied is electroweak precision data (EWPD) where contributions to the $U$ parameter first arise at dimension-8 [21]. Formally the dimension- 6 operators are the leading terms in the EFT expansion. However there are various scenarios in which this is not the case practically speaking. Perhaps the most obvious among these is when the interference between the dimension- 6 amplitude and the SM amplitude is suppressed or even vanishes. Additionally there could be a difference in the experimental precision of the measurements being considered [22]. Finally, we comment on the structure the renormalization group evolution (RGE) equations of the dimension- 8 operators.

The rest of the paper is organized as follows. Section 2 lays down the notation and conventions we use, including the semantics of number of operators versus number of types of operators. We then discuss how we performed the operator classification in section 3 with the results given in section 4 . We briefly explore light-by-light scattering, EWPD, as well as models involving scalar $\mathrm{SU}(2)_{w}$ quartets where there is interesting interplay between dimension- 6 and dimension- 8 effects in section 5 . Additionally we comment on the renormalization group evolution (RGE) of the dimension- 8 operators in section 6 before concluding in section 7 . For convenience we provide tables of dimension- 6 and -7 operators in appendix A. 


\section{Notation and conventions}

We start by considering the various uses of the word operator. See ref. [23] for further discussion. We a define operator to be a gauge and Lorentz invariant contraction of fields and derivatives with specific flavor indices. A Lagrangian term, or just term or short, collects all of operators with the same gauge and Lorentz structure into a single unit, i.e. a term collapses the flavor indices of otherwise identical operators. By construction, a Lagrangian term of mass dimension $d \leq 8$ may contain no more than $n_{g}^{4}$ operators. $^{2}$ Ref. [23] defines a type of operator as the collection of terms with the combination of fields (and derivatives) with conjugate counted separately. In this work we use a broader definition of a type of operator where the conjugate fields are counted in unison with the unconjugated fields. Our types of operators are therefore supersets of those in [23], of which there are 541 to our 231. This definition of a type of operator allows us systematically label the operators in a phenomenologically friendly way. The largest set of operators we consider is a class where the operators are grouped by the number of fields of a given spin as well as the number of derivatives. It is useful to consider subclasses when discussing the RGE of the dimension- 8 operators. Subclasses treat conjugate fields separately. For example, class 1 has three subclasses, $\left\{X_{L}^{4}, X_{L}^{2} X_{R}^{2}, X_{R}^{4}\right\} \in X^{4}$, and class 18 also has three subclasses, $\left\{\psi^{4} H^{2}, \psi^{2} \bar{\psi}^{2} H^{2}, \bar{\psi}^{4} H^{2}\right\} \in \psi^{4} H^{2}$. We tolerate a slight abuse of notation between classes and subclasses relying on context to distinguish which set is being discussed.

Moving onto physics conventions, the SM Lagrangian is given by

$$
\begin{aligned}
\mathcal{L}_{\mathrm{SM}}= & -\frac{1}{4} \sum_{X} X_{\mu \nu} X^{\mu \nu}+\left(D_{\mu} H^{\dagger}\right)\left(D^{\mu} H\right)+\sum_{\psi} \bar{\psi} i \not D \psi \\
& -\lambda\left(H^{\dagger} H-\frac{v^{2}}{2}\right)^{2}-\left[H_{j}^{\dagger} \bar{d} Y_{d} q^{j}+\widetilde{H}_{j}^{\dagger} \bar{u} Y_{u} q^{j}+H_{j}^{\dagger} \bar{e} Y_{e} l^{j}+\text { h.c. }\right] .
\end{aligned}
$$

In eq. (2.1), and throughout this work, we generically refer to field strengths as $X=$ $\left\{G^{A}, W^{I}, B\right\}$, and to fermions as $\psi=\{l, e, q, u, d\}$.

The gauge covariant derivative is

$$
\left(D_{\mu} q\right)^{j \alpha}=\left(\left(\partial_{\mu}+i g_{1} \mathrm{y} B_{\mu}\right) \delta_{\beta}^{\alpha} \delta_{k}^{j}+i g_{2}\left(t^{I}\right)_{k}^{j} W_{\mu}^{I} \delta_{\beta}^{\alpha}+i g_{3}\left(T^{A}\right)_{\beta}^{\alpha} A_{\mu}^{A} \delta_{k}^{j}\right) q^{k \beta},
$$

where the generators of $\mathrm{SU}(3)_{c}$ and $\mathrm{SU}(2)_{w}$ are $T^{a}$ and $t^{I}=\tau^{I} / 2$, respectively. The $\mathrm{U}(1)_{y}$ hypercharge is given by y with $Q=\tau^{3}+\mathrm{y}$. For $\mathrm{SU}(3)_{c}$ fundamental and adjoint indices are denoted $\alpha, \beta, \gamma$ and $A, B, C$, respectively, while for $\mathrm{SU}(2)_{w}$ the fundamental and adjoint indices are respectively labeled $j, k, m$ and $I, J, K$.

Anti-symmetrization of indices is denoted by a pair of square brackets, $[\mu \nu]$, and symmetrization is denoted by a pair of round brackets, $(\mu \nu)$. The definition of $\widetilde{H}$ is

$$
\widetilde{H}_{j}=\epsilon_{j k} H^{\dagger k}
$$

where $\epsilon_{j k}=\epsilon_{[j k]}$ is the $\mathrm{SU}(2)$ invariant tensor with $\epsilon_{12}=1$. The dual field strength is defined as

$$
\widetilde{X}_{\mu \nu}=\frac{1}{2} \epsilon_{\mu \nu \rho \sigma} X^{\rho \sigma}
$$

with $\epsilon_{0123}=1$.

\footnotetext{
${ }^{2}$ Starting at dimension- $9 n_{g}^{6}$ is possible.
} 
We will sometimes refer to the following combinations of field strength as they are typically what are used when counting coefficients,

$$
X_{L, R}^{\mu \nu}=\frac{1}{2}\left(X^{\mu \nu} \mp i \widetilde{X}^{\mu \nu}\right) .
$$

These field strengths have simple Lorentz transformation properties, $X_{L} \sim(1,0), X_{R} \sim$ $(0,1)$ under $\mathrm{SU}(2)_{L} \otimes \mathrm{SU}(2)_{R}$. Similarly $l$ and $q$ are left-handed fermion fields, whereas $e, u$, and $d$ are right-handed fields. When necessary Lorentz indices in the fundamental representations are indicated by $a, b, \dot{a}, \dot{b}$, e.g. $q_{L} \sim\left(q_{L}\right)_{a}, B_{R} \sim\left(B_{R}\right)_{(\dot{a} \dot{b})}$.

The SMEFT extends the SM by adding all of the higher-dimensional operator that are gauge invariant under the SM with the caveat that redundant operators should not be included

$$
\mathcal{L}_{\mathrm{SMEFT}}=\mathcal{L}_{\mathrm{SM}}+\sum_{d>4} \mathcal{L}^{(d)}
$$

For the dimension- 6 operators we keep notation that has been well-established in the literature, see e.g. [5]. On the other hand, we use a systematic, if at times cumbersome, notation for labelling the operators of mass-dimension 7 and above. For types of operators with a single Lagrangian term we label them as follows

$$
\mathcal{L}^{(d)} \supset \sum_{\text {type }} C_{\text {type }} Q_{\text {type }}, \quad n_{\text {term }}=1,
$$

where $n_{\text {term }}$ is the number of terms of a given type. The type of operator is denoted as the fields and the derivatives in the operator raised to the power of the number of times that type of object appears in the operator, e.g. the label $l e B H^{3}$ indicates that this term has one left-handed lepton field, one right-handed electron field, one hypercharge field strength, and three Higgs fields. If a type of operator has multiple terms, not counting Hermitian conjugates, we instead label the operators as

$$
\mathcal{L}^{(d)} \supset \sum_{\text {type }} \sum_{i=1}^{n_{\text {term }}} C_{\text {type }}^{(i)} Q_{\text {type }}^{(i)}, \quad n_{\text {term }}>1 .
$$

Consider as an explicit example,

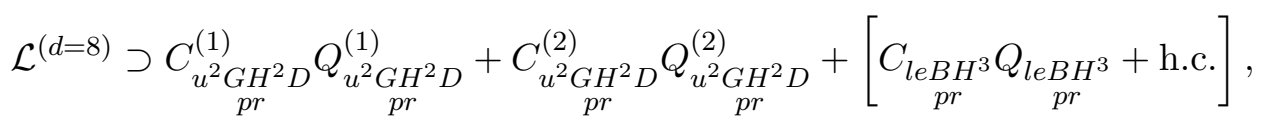

with

$$
\begin{aligned}
Q_{u^{2} G H^{2} D}^{(1)} & =\left(\bar{u}_{p} \gamma^{\nu} T^{A} u_{r}\right) D^{\mu}\left(H^{\dagger} H\right) G_{\mu \nu}^{A}, \\
Q_{u^{2} G H^{2} D}^{(2)} & =\left(\bar{u}_{p} \gamma^{\nu} T^{A} u_{r}\right) D^{\mu}\left(H^{\dagger} H\right) \widetilde{G}_{\mu \nu}^{A}, \\
Q_{l e B H^{3}} & =\left(\bar{l}_{p} \sigma^{\mu \nu} e_{r}\right) H\left(H^{\dagger} H\right) B_{\mu \nu} .
\end{aligned}
$$

Flavor indices explicitly appear in eq. (2.9). Fermion fields have a flavor index $p, r, s, t$ that runs over 1, 2, 3 for three generations. The fermion fields themselves are in the weak eigenstate basis. The Yukawa matrices, $Y_{e, u, d}$, in eq. (2.1) are matrices in flavor space. 
Note that we do not explicitly label the transpose of a spinor in fermion bilinears involving a charge conjugation operator, e.g. $\psi_{1} C \psi_{2} \equiv \psi_{1}^{T} C \psi_{2}, \psi_{1} C \sigma_{\mu \nu} \psi_{2} \equiv \psi_{1}^{\top} C \sigma_{\mu \nu} \psi_{2}$. Finally, it is convenient to define Hermitian derivatives e.g.

$$
\begin{aligned}
i H^{\dagger} \overleftrightarrow{D}_{\mu} H & =i H^{\dagger}\left(D_{\mu} H\right)-i\left(D_{\mu} H^{\dagger}\right) H, \\
i H^{\dagger} \overleftrightarrow{D}_{\mu}^{I} H & =i H^{\dagger} \tau^{I}\left(D_{\mu} H\right)-i\left(D_{\mu} H^{\dagger}\right) \tau^{I} H
\end{aligned}
$$

\section{Operator classification}

Having defined our notation in section 2 we can more precisely state our goal. We are trying to find the minimum number of Lagrangian terms needed to give all of the operators at dimension- 8 subject to the constraint that no term may contain more than $n_{g}^{4}$ operators. For bosonic and two-fermion operators this constraint is trivially satisfied as those terms always contain one and $n_{g}^{2}$ operators, respectively. Four-fermion operators where two or more of the fields are identical constitute the interesting cases.

We use existing results from the literature when they are available. All of the bosonic operators have been classified previously [18-20]. Ref. [19] also gave partial results for three of the two-fermion classes that were sufficient to allow us to deduce the remaining operators in those classes. As this work was being finalized refs. [24, 25] appeared, which classified a subset of four-fermion operators with two derivatives. However there are still non-trivial results for us to work out in that class.

When classifying the dimension- 8 operators we exploit the fact that not only are the types of operators known, but the number of operators is also known, see ref. [10]. In particular, we leverage the Python package BasisGen [13], which we use to get the number of operators for each type of operator. Additionally, we use the Mathematica program Sym2Int [11], which not only gives the number of operators per type, but also the flavor representations when there are identical particles in the operator. Furthermore, Sym2Int gives the number of Lagrangian terms per type of operator except when the operator contains both derivatives and identical particles. In that case a range is given because the permutation symmetry of operators with derivatives is ambiguous due to integration by parts (IBP) redundancies.

Ref. [23] used Sym2Int to derive lower and upper limits on the number of terms of dimension-8 operators, $1025 \leq N_{\text {term }} \leq 1102$. Our basis contains 1031 terms, close to the lower limit. The counting is clear from [10] for that one generation of fermions there are 993 terms. Of the remaining 38 terms we identified, all of which vanish in the absence of flavor structure, six of them involve derivatives. For each of these six terms there is another term of the same type that does not vanish in the absence of flavor structure, which is consistent with the ambiguity originating from terms with derivatives. In principle this could allow us to reduce the number of terms in our basis and hit the lower limit. However, the total number of operators contained in four of the terms is $n_{g}^{4}+\frac{1}{2} n_{g}^{3}\left(n_{g}-1\right)$, which exceeds the maximum of operators that can be placed in a single term, $n_{g}^{4}$. Therefore these four potentially redundant terms do need to be retained as independent terms in the Lagrangian. Finally the last term plus its Hermitian conjugate have a different Lorentz structure than 
the other term (+h.c.) of the same type that does not vanish in the absence of flavor structure, making it an independent term as well.

Beyond getting the number of terms correct we need to ensure that the operators in our basis are independent. Operators with derivatives can be related through integration by parts. When there are multiple derivatives care must be taken to select operators for the basis that span the entire space of possible operators for that class. See the discussion of class 16 below for an example of this. Operators can also be related to each other through the equations of motion (EOM). We use the EOM the remove redundant terms, trading them for basis operators in the same class, operators with fewer derivatives, and sometimes operators of lower mass dimension. See the discussion of class 17 below for an example of this, and see e.g. [26] for the SM equations of motion. Our basis does not explicitly contain an EOM. Operators with derivatives can be IBP, and some of the resulting terms contain an EOM. However it is never the case that all of the resulting terms have an EOM. Lastly, there are various tensor and spinor identities that relate operators to each other. There are the Fierz identities, for example for $\mathrm{SU}(2)$

$$
\left(\tau^{I}\right)_{j}^{k}\left(\tau^{I}\right)_{m}^{n}=2 \delta_{j}^{n} \delta_{m}^{k}-\delta_{j}^{k} \delta_{m}^{n}
$$

There are identities involving the Levi-Civita symbol, e.g. in two-dimensions

$$
\epsilon_{j k} \epsilon_{m n}+\epsilon_{j m} \epsilon_{n k}+\epsilon_{j n} \epsilon_{k m}=0
$$

There are identities for products of Dirac matrices, e.g. the anti-symmetric Dirac tensor is self-dual

$$
\epsilon^{\rho \tau \mu \nu} \sigma_{\mu \nu} P_{R}=2 i \sigma^{\rho \tau} P_{R}
$$

\subsection{Bosonic operators}

1. $X^{4}$.

The $X^{4}$ operators for a single Yang-Mills field were classified in ref. [18]. Ref. [20] generalized this result to the SM field content. Note that dimension- 8 is the lowest dimension where a subclass of operators contains both $X_{L}$ and $X_{R}$.

2. $H^{8}$.

$\left(H^{\dagger} H\right)^{4}$ is the only possibility.

3. $H^{6} D^{2}$.

The $H^{6} D^{2}$ operators were classified in ref. [19].

4. $H^{4} D^{4}$.

Both refs. [19] and [20] classified the $H^{4} D^{4}$ operators.

5. $X^{3} H^{2}$.

The $X^{3} H^{2}$ operators were classified in ref. [19]. Note that the two terms in $Q_{W^{2} B H^{2}}^{(2)}$ are equivalent via the identity

$$
\widetilde{X}_{\mu \rho} Y^{\rho \nu}=-X^{\nu \rho} \widetilde{Y}_{\rho \mu}-\frac{1}{2} X_{\alpha \beta} \tilde{Y}^{\alpha \beta} \delta_{\mu}^{\nu}
$$


For backwards compatibility we construct our basis with $Q_{W^{2} B H^{2}}^{(2)}$ as originally defined by ref. [19] as opposed to, say, only keeping the second term.

6. $X^{2} H^{4}$.

The $X^{2} H^{4}$ operators were classified in ref. [19].

7. $X^{2} H^{2} D^{2}$.

Both refs. [19] and [20] classified the $X^{2} H^{2} D^{2}$ operators.

8. $X H^{4} D^{2}$.

The $X H^{4} D^{2}$ operators were classified in ref. [19].

\section{$3.2 \quad$ Two-fermion operators}

9. $\psi^{2} X^{2} H$.

For the dimension- 8 class $\psi^{2} X^{2} H, 24$ terms arise from joining a field strength to a dimension- 6 operator of the form $\psi^{2} X H$, whereas 48 terms come from the product of two field strengths and a Yukawa interaction, $\left(X^{2}\right)\left(\psi^{2} H\right)$. See table 21 for the dimension- 6 operators.

10. $\psi^{2} X H^{3}$.

In the class $\psi^{2} X H^{3}, 16$ of the 22 terms are identical to the dimension- 6 terms $\psi^{2} X H$ up to an extra factor of $\left(H^{\dagger} H\right)$. The remaining six terms, all involving $W_{\mu \nu}^{I}$, instead have the dimension-2 covariant $\left(H^{\dagger} \tau^{I} H\right)$.

11. $\psi^{2} H^{2} D^{3}$.

Ref. [19] classified the four terms involving $q^{2} H^{2} D^{3}$. The remaining 12 terms in the class can be deduced from the results of ref. [19].

12. $\psi^{2} H^{5}$.

The class $\psi^{2} H^{5}$ is identical to the dimension- 6 class $\psi^{2} H^{3}$ up to an extra factor of $\left(H^{\dagger} H\right)$.

13. $\psi^{2} H^{4} D$.

Ref. [19] classified the four operators involving $q^{2} H^{4} D$. The term $Q_{q^{2} H^{4} D}^{(2)}$ contains a sum

$$
Q_{q^{2} H^{4} D}^{(2)}=Q_{q^{2} H^{4} D}^{(2 l)}+Q_{q^{2} H^{4} D}^{(2 r)} .
$$

The term $Q_{q^{2} H^{4} D}^{(3)}$ is related to the righthand side of eq. (3.5) as follows

$$
i Q_{q^{2} H^{4} D}^{(3)}=-Q_{q^{2} H^{4} D}^{(2 l)}+Q_{q^{2} H^{4} D}^{(2 r)}
$$

This can be seen using the following variation of eq. (3.1)

$$
\delta_{j}^{k}\left(\tau^{I}\right)_{m}^{n}-\left(\tau^{I}\right)_{j}^{k} \delta_{m}^{n}=i \epsilon^{I J K}\left(\tau^{J}\right)_{j}^{n}\left(\tau^{K}\right)_{m}^{k} .
$$

As was the case with the class 5 operator $Q_{W^{2} B H^{2}}^{(2)}$ we choose to keep $Q_{q^{2} H^{4} D}^{(2)}$ and $Q_{q^{2} H^{4} D}^{(3)}$ is our basis as opposed to their summands for backwards compatibility. The remaining nine terms in the class can be deduced from the results of ref. [19]. 
14. $\psi^{2} X^{2} D$.

We use integration by parts to place the derivative on a fermion field. Then in order for the operator to not be "reduced" to a class with fewer derivatives through the use of the equations of motion the fermionic component of the operator must not be Lorentz invariant. Class 7 also contains two field strengths, see above, and a subset of the operators in class 7 have covariants formed from Higgs fields and derivatives that transform as $(1,1)$ under $\mathrm{SU}(2)_{L} \otimes \mathrm{SU}(2)_{R}$. We take the field strength components of that subset of class 7 operators and use them for the class 14 operators, contracting them with fermionic covariants of the form $\bar{\psi} \gamma^{\mu} D^{\nu} \psi$. In particular, we use $Q_{G^{2} H^{2} D^{2}}^{(1)}$ as the template for when a fermion is not charged under a gauge group, $Q_{W^{2} H^{2} D^{2}}^{(1,4-6)}$ when it is charged under a gauge group, and $Q_{W B H^{2} D^{2}}^{(1,4-6)}$ when it is charged under two gauge groups. Three terms are not covered by this procedure. They involve quarks and two gluon field strength where the $\mathrm{SU}(3)_{c}$ adjoint indices are contracted with the symmetric $d^{A B C}$ symbol.

15. $\psi^{2} X H^{2} D$.

Ref. [19] classified the 12 terms involving $q^{2} W H^{2} D$. Of the remaining 74 terms, 68 of them have a form analogous to those classified by ref. [19]. The final six terms in the class are instead analogous to the dimension-6 operator $Q_{H u d}$ with the addition of a field strength.

16. $\psi^{2} X H D^{2}$.

Things become more complicated when there are two or more derivatives in the operator. As such it is useful to introduce some additional machinery to classify the operators. We use the procedure given in ref. [7] for removing terms that are reducible through the use of the equations of motion. In a nutshell, the procedure says Lorentz indices should be symmetrized for representations that are triplets or higher under either $\mathrm{SU}(2)_{L}$ or $\mathrm{SU}(2)_{R}$. In terms of Lorentz indices we have for example

$$
D \psi_{L} \sim\left(D \psi_{L}\right)_{(a b), \dot{a}}, \quad D X_{R} \sim\left(D X_{R}\right)_{a,(\dot{a} \dot{b} \dot{c})}, \quad D^{2} H \sim\left(D^{2} H\right)_{(a b),(\dot{a} \dot{b})} .
$$

We now work through a representative example with field content $\bar{l}, e, H, B_{L}$ following the procedure laid out in ref. [19]. Using relations like those in (3.8) we see that operators with a derivative acting on the field strength or two derivatives acting on a fermion can be reduced using the EOM. Ignoring for the time being constraints from IBP this leaves us with four possibilities

$$
\begin{aligned}
& x_{1}=(D \bar{l})_{a,(\dot{a} \dot{c})} e_{\dot{d}}(D H)_{b, \dot{b}} B_{(c d)} \epsilon^{a c} \epsilon^{b d} \epsilon^{\dot{a} \dot{d}} \epsilon^{\dot{c} \dot{b}}, \\
& x_{2}=\bar{l}_{\dot{c}}(D e)_{a,(\dot{a} \dot{d})}(D H)_{b, \dot{b}} B_{(c d)} \epsilon^{a c} \epsilon^{b d} \epsilon^{\dot{a} \dot{c}} \epsilon^{\dot{d} \dot{b}}, \\
& x_{3}=(D \bar{l})_{a,(\dot{a} \dot{c})}(D e)_{b,(\dot{b} \dot{d})} H B_{(c d)} \epsilon^{a c} \epsilon^{b d} \epsilon^{\dot{a} \dot{b}} \epsilon^{\dot{c} \dot{d}}, \\
& x_{4}=\bar{l}_{\dot{c}} e_{\dot{d}}\left(D^{2} H\right)_{(a b),(\dot{a} \dot{b})} B_{(c d)} \epsilon^{a c} \epsilon^{b d} \epsilon^{\dot{a} \dot{c} \dot{c}} \epsilon^{\dot{b} \dot{d}},
\end{aligned}
$$

where we have not shown the $\mathrm{SU}(2)_{w}$ contraction as it is trivial. 
To determine redundancies coming from integration by parts we need operators transforming as $\left(\frac{1}{2}, \frac{1}{2}\right)$ under the Lorentz group with one fewer derivative than the operators of interest. There are three possibilities in this example

$$
\begin{aligned}
& y_{1}=(D \bar{l})_{a,(\dot{a} \dot{c})} e_{\dot{d}} H B_{(c d)} \epsilon^{a c} \epsilon^{\dot{a} \dot{d}}, \\
& y_{2}=\bar{l}_{\dot{c}}(D e)_{a,(\dot{a} \dot{d})} H B_{(c d)} \epsilon^{a c} \epsilon^{\dot{a} \dot{c}}, \\
& y_{3}=\bar{l}_{\dot{c}} e_{\dot{d}}(D H)_{a, \dot{a}} B_{(c d)} \frac{1}{2} \epsilon^{a c}\left(\epsilon^{\dot{a} \dot{c}}+\epsilon^{\dot{a} \dot{d}}\right) .
\end{aligned}
$$

The derivatives of the $y_{i}$ show which of the $x_{i}$ are related by IBP

$$
\begin{aligned}
& D_{b, \dot{b}} y_{1}=x_{1}+x_{3}=0, \\
& D_{b, \dot{b}} y_{2}=x_{2}+x_{3}=0, \\
& D_{b, \dot{b}} y_{3}=\frac{1}{2} x_{1}+\frac{1}{2} x_{2}+x_{4}=0,
\end{aligned}
$$

where the appropriate contraction of the remaining Lorentz indices in the leftmost terms is understood. By inspection of (3.11) we see that any of the four candidate operator can be transformed into any of the remaining three through the use of IBP.

We repeat the same procedure, omitting the details here, to find the class 17 operators with field content $\bar{l}, e, H, B_{R}$. In this case there are eight candidate operators, $x_{i}$, and six operators in the Lorentz four-vector representation, $y_{i}$. Given the larger number of operators in this case we use Mathematica to solve the system of constraints, yielding two operators.

We are left with a total of three $Q_{l e H B D^{2}}$ terms (+h.c.)

$$
\begin{aligned}
& \epsilon^{a c} \epsilon^{b d} \epsilon^{\dot{a} \dot{b}} \epsilon^{\dot{c}} \dot{l}_{\dot{a}}(D e)_{a,(\dot{b} \dot{c})}(D H)_{b, \dot{d}}\left(B_{L}\right)_{c d}, \\
& \epsilon^{a b} \epsilon^{\dot{a} \dot{a}} \epsilon^{\dot{c} \dot{e}} \epsilon^{\dot{d} \dot{f}} \bar{l}_{\dot{a}}(D e)_{a,(\dot{b} \dot{c})}(D H)_{b, \dot{d}}\left(B_{R}\right)_{(\dot{e} \dot{f})}, \\
& \epsilon^{a b} \epsilon^{\dot{a} \dot{d}} \epsilon^{\dot{b} \dot{e}} \epsilon^{\dot{c} \dot{f}} \bar{l}_{\dot{a}} e_{\dot{b}}(D H)_{a, \dot{c}}\left(D B_{R}\right)_{b,(\dot{d} \dot{e} \dot{f})}
\end{aligned}
$$

which matches the counting we found using BasisGen. Translating the Lorentz contractions from $\mathrm{SU}(2)_{L} \otimes \mathrm{SU}(2)_{R}$ to $\mathrm{SO}(3,1)$ and translating $B_{L}$ and $B_{R}$ to $B$ and $\widetilde{B}$ we find

$$
\begin{aligned}
Q_{l e B H D^{2}}^{(1)} & =\left(\bar{l}_{p} \sigma^{\mu \nu} D^{\rho} e_{r}\right)\left(D_{\nu} H\right) B_{\rho \mu} \\
Q_{l e B H D^{2}}^{(2)} & =\left(\bar{l}_{p} D^{\rho} e_{r}\right)\left(D^{\nu} H\right) \widetilde{B}_{\rho \nu} \\
Q_{l e B H D^{2}}^{(3)} & =\left(\bar{l}_{p} \sigma^{\mu \nu} e_{r}\right)\left(D^{\rho} H\right)\left(D_{\rho} B_{\mu \nu}\right) .
\end{aligned}
$$

The remaining 42 terms in class 17 can be deduced from the $Q_{l e H B D^{2}}$ operators in $(3.13)+$ h.c..

17. $\psi^{2} H^{3} D^{2}$.

Starting with relations like those in (3.8) we see that any operator in this class with two derivatives acting on the same field can be reduced using the EOM. Therefore 
an operator with one derivative on each of the two fermion fields can be traded for an operator with one derivative on a Higgs field, one derivative on a fermion plus operators with fewer derivatives. From here we extend the results of ref. [2] to move any remaining derivatives acting on fermions onto Higgs fields, again plus operators with fewer derivatives. The first relation is

$$
\begin{aligned}
H^{\dagger} H\left(D_{\mu} H\right) \bar{\psi} \sigma^{\mu \nu} D_{\nu} \psi & =\frac{i}{2} H^{\dagger} H\left(D_{\mu} H\right) \bar{\psi}\left(\gamma^{\mu} \not D-\not D \gamma^{\mu}\right) \psi \\
& =i H^{\dagger} H\left(D_{\mu} H\right) \bar{\psi} \gamma^{\mu} \not D \psi-i H^{\dagger} H\left(D_{\mu} H\right) \bar{\psi} D^{\mu} \psi \\
& =-i H^{\dagger} H\left(D_{\mu} H\right) \bar{\psi} D^{\mu} \psi+\psi^{2} H^{4} D+E
\end{aligned}
$$

where $E$ represents operators that vanish via the EOM. The other relation we need is

$$
\begin{aligned}
2 H^{\dagger} H\left(D_{\mu} H\right) \bar{\psi} D^{\mu} \psi= & H^{\dagger} H\left(D_{\mu} H\right) \bar{\psi}\left(\gamma^{\mu} \not D+\not D \gamma^{\mu}\right) \psi \\
= & \left(H^{\dagger} H\left(D_{\mu} H\right) \bar{\psi} \gamma^{\mu} \not D \psi-H^{\dagger} H\left(D_{\mu} H\right)(\not D \bar{\psi}) \gamma^{\mu} \psi\right. \\
& \left.\quad-D_{\nu}\left[H^{\dagger} H\left(D_{\mu} H\right)\right] \bar{\psi} \gamma^{\nu} \gamma^{\mu} \psi+T\right) \\
= & -D_{\nu}\left[H^{\dagger} H\left(D_{\mu} H\right)\right] \bar{\psi} \gamma^{\nu} \gamma^{\mu} \psi+E+T
\end{aligned}
$$

where $T$ stands for a total derivative.

After all this we are left with six terms +h.c. where the derivatives act only on Higgs fields. In particular, having already established that operators in this class cannot have two derivatives acting on the same field, the derivatives can either act on $H$ and $H^{\dagger}$ or there can be one derivative on each of the $H$ fields. For each of these cases the fermion pair can either be in the $(0,0)$ or $(0,1)$ representation of the Lorentz group. (The classification for the Hermitian conjugate operators proceeds in an identical fashion.) Finally when the derivatives act on $H$ and $H^{\dagger}$ the covariants can either be $\mathrm{SU}(2)_{w}$ singlets or adjoints. The same logic applies for all three choices for the pair of fermions.

\subsection{Four-fermion operators}

18. $\psi^{4} H^{2}$.

All 38 of the $\psi^{4}$ dimension- 6 terms can be multiplied by $\left(H^{\dagger} H\right)$. Focusing on $B$ preserving operators, an additional 23 terms are formed by inserting a $\tau^{I}$ into a $\psi^{4}$ operator (with at least two left-handed fermions) and joining it to the dimension-2 covariant $\left(H^{\dagger} \tau^{I} H\right)$. Operators of the type $Q_{l^{2} q^{2} H^{2}}$ provide three of the these terms, whereas all other types of operators provide one term (+h.c.). There are also 22 $\psi^{4} H^{2}$ terms that, schematically, are products of Yukawa interactions, $(\bar{L} R H)(\bar{L} R H)$ or $(\bar{L} R H)\left(H^{\dagger} \bar{R} L\right)$. Among these there is one new type of operator, $Q_{l^{2} u d H^{2}}$. BiYukawa terms with four fields that are fundamentals under either $\mathrm{SU}(2)_{w}$ or $\mathrm{SU}(3)_{c}$ where two of those fields are identical can be contracted either as singlets or adjoints. 
There are some redundant operators involving identical left-handed fermions. For example, the terms

$$
\begin{aligned}
Q_{l^{4} H^{2}}^{(3)} & =\left(\bar{l}_{p} \gamma^{\mu} \tau^{I} l_{r}\right)\left(\bar{l}_{s} \gamma_{\mu} \tau^{I} l_{t}\right)\left(H^{\dagger} H\right), \\
Q_{l^{4} H^{2}}^{(4)} & =\left(\bar{l}_{p} \gamma^{\mu} \tau^{I} l_{r}\right)\left(\bar{l}_{s} \gamma_{\mu} l_{t}\right)\left(H^{\dagger} \tau^{I} H\right), \\
Q_{l^{4} H^{2}}^{(5)} & =\epsilon^{I J K}\left(\bar{l}_{p} \gamma^{\mu} \tau^{I} l_{r}\right)\left(\bar{l}_{s} \gamma_{\mu} \tau^{J} \tau^{I} l_{t}\right)\left(H^{\dagger} \tau^{K} H\right),
\end{aligned}
$$

are related to the operators in our basis

$$
\begin{aligned}
Q_{l^{4} H^{2}}^{(3)} & =\underset{l^{4} H^{2}}{p r s t}-Q_{l^{4} H^{2}}^{(1)}, \\
Q_{l^{4} H^{2}}^{(1)} & =Q_{l^{4} H^{2}}^{(2)}, \\
Q_{p r s t}^{(4)} & \text { stpr } \\
i Q_{l^{4} H^{2}}^{(5)} & =\underset{l^{4} H^{2}}{(2)}-Q_{l^{4} H^{2}}^{(2)} . \\
{ }_{\text {prst }}^{(2)} & { }_{\text {stpr }}
\end{aligned}
$$

This can be seen using eqs. (3.1) and (3.7).

The terms for baryon number violating operators with dimension- 6 analogs follow the logic laid out for the $B$ operators. However there are two interesting flavor structures. The dimension-6 operator $Q_{q q u e}$ is symmetric in its $q$ flavor indices [4]. This constraint is broken by the additional $\mathrm{SU}(2)_{w}$ in $Q_{e q^{2} u H^{2}}$, giving it full flavor rank, $n_{g}^{4}$. The dimension-6 operator $Q_{q q q l}$ also has a flavor constraint [4]

$$
\underset{p r s t}{Q_{q q q l}}+\underset{r p s t}{Q_{q q q l}}=\underset{\text { sprt }}{Q_{q q q l}}+\underset{s r p t}{Q_{q q q l}}
$$

which can be derived using eq. (3.2). The operators $Q_{l q^{3} H^{2}}^{(1,2)}$ respect this constraint, leading to each of their Lagrangian terms (+h.c.) containing $\frac{2}{3} n_{g}^{2}\left(2 n_{g}^{2}+1\right)$ operators. On the other hand, $Q_{l q^{3} H^{2}}^{(3)}$ has mixed symmetry. It is symmetric in $p$ and $r$ and antisymmetric in $r$ and $s$, which causes it to vanish when there is only when generation of fermions. As a result of the six fundamental $\mathrm{SU}(2)_{w}$ indices there are eight redundant operators

$$
\begin{aligned}
& Q_{l q^{3} H^{2}}^{(1 a)}=\epsilon_{\alpha \beta \gamma} \epsilon_{m j} \epsilon_{k n}\left(q_{p}^{m \alpha} C q_{r}^{j \beta}\right)\left(q_{s}^{k \gamma} C l_{t}^{n}\right)\left(H^{\dagger} H\right), \\
& Q_{l q^{3} H^{2}}^{(1 b)}=\epsilon_{\alpha \beta \gamma}\left(\tau^{I} \epsilon\right)_{m j}\left(\tau^{I} \epsilon\right)_{k n}\left(q_{p}^{m \alpha} C q_{r}^{j \beta}\right)\left(q_{s}^{k \gamma} C l_{t}^{n}\right)\left(H^{\dagger} H\right), \\
& Q_{l q^{3} H^{2}}^{(2 a)}=\epsilon_{\alpha \beta \gamma} \epsilon_{m j}\left(\tau^{I} \epsilon\right)_{k n}\left(q_{p}^{m \alpha} C q_{r}^{j \beta}\right)\left(q_{s}^{k \gamma} C l_{t}^{n}\right)\left(H^{\dagger} \tau^{I} H\right), \\
& Q_{l q^{3} H^{2}}^{(2 b)}=\epsilon_{\alpha \beta \gamma}\left(\tau^{I} \epsilon\right)_{j n} \epsilon_{k m}\left(q_{p}^{m \alpha} C q_{r}^{j \beta}\right)\left(q_{s}^{k \gamma} C l_{t}^{n}\right)\left(H^{\dagger} \tau^{I} H\right), \\
& Q_{l q^{3} H^{2}}^{(3 a)}=\epsilon_{\alpha \beta \gamma}\left(\tau^{I} \epsilon\right)_{m j} \epsilon_{k n}\left(q_{p}^{m \alpha} C q_{r}^{j \beta}\right)\left(q_{s}^{k \gamma} C l_{t}^{n}\right)\left(H^{\dagger} \tau^{I} H\right), \\
& Q_{l q^{3} H^{2}}^{(3 b)}=\epsilon_{\alpha \beta \gamma} \epsilon_{j n}\left(\tau^{I} \epsilon\right)_{k m}\left(q_{p}^{m \alpha} C q_{r}^{j \beta}\right)\left(q_{s}^{k \gamma} C l_{t}^{n}\right)\left(H^{\dagger} \tau^{I} H\right), \\
& Q_{l q^{3} H^{2}}^{(4 a)}=\epsilon_{\alpha \beta \gamma} \epsilon^{I J K}\left(\tau^{I} \epsilon\right)_{m n}\left(\tau^{J} \epsilon\right)_{j k}\left(q_{p}^{m \alpha} C q_{r}^{j \beta}\right)\left(q_{s}^{k \gamma} C l_{t}^{n}\right)\left(H^{\dagger} \tau^{K} H\right), \\
& Q_{l q^{3} H^{2}}^{(4 b)}=\epsilon_{\alpha \beta \gamma} \epsilon^{I J K}\left(\tau^{I} \epsilon\right)_{m j}\left(\tau^{J} \epsilon\right)_{k n}\left(q_{p}^{m \alpha} C q_{r}^{j \beta}\right)\left(q_{s}^{k \gamma} C l_{t}^{n}\right)\left(H^{\dagger} \tau^{K} H\right) .
\end{aligned}
$$


The operators in (3.19) can be written in terms of the operators in our basis using eq. (3.1), eq. (3.7), and the following relations obtained from eq. (3.1)

$$
\begin{aligned}
\epsilon_{m j}\left(\tau^{I} \epsilon\right)_{k n}+\left(\tau^{I} \epsilon\right)_{m j} \epsilon_{k n} & =\epsilon_{m n}\left(\tau^{I} \epsilon\right)_{j k}-\left(\tau^{I} \epsilon\right)_{m n} \epsilon_{j k}, \\
i \epsilon^{I J K}\left[\left(\tau^{J} \epsilon\right)_{m n}\left(\tau^{K} \epsilon\right)_{j k}-\frac{1}{2}\left(\tau^{J} \epsilon\right)_{m j}\left(\tau^{K} \epsilon\right)_{k n}\right] & =\epsilon_{m n}\left(\tau^{I} \epsilon\right)_{j k}+\left(\tau^{I} \epsilon\right)_{m n} \epsilon_{j k} .
\end{aligned}
$$

In particular, the relations are

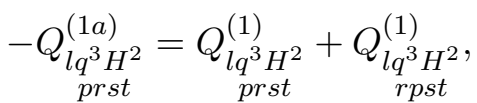

$$
\begin{aligned}
& -Q_{p r s t}^{(1 b)}=\underset{p r s t}{q_{l q^{3} H^{2} H^{2}}^{(1)}}-\underset{r p s t}{Q_{l q^{3} H^{2}}^{(1)}} \\
& Q_{l q^{3} H^{2}}^{(2 b)}=Q_{l q^{3} H^{2}}^{(2)}, \\
& Q_{\underset{p r s t}{(3 b)}}^{\left(q^{3} H^{2}\right.}=-\underset{l q^{3} H^{2}}{r p s t},
\end{aligned}
$$

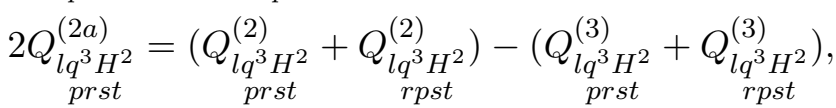

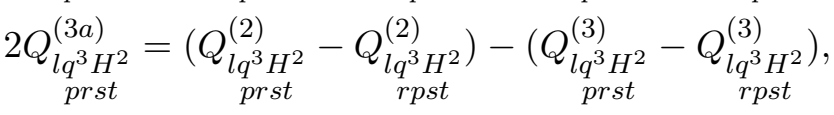

$$
\begin{aligned}
& \underset{p r s t}{Q_{l q^{3} H^{2}}^{(4 a)}}=\underset{\text { rpst }}{Q_{l q^{3} H^{2}}^{(2)}}-\underset{r p s t}{Q_{l q^{3} H^{2}}^{(3)}}
\end{aligned}
$$

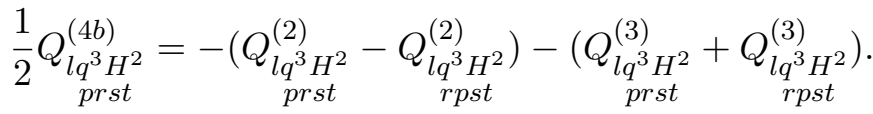

In addition to $\mathbb{B}$ operators with dimension- 6 analogs, three new types of operators appear. These were three of the types of operators identified by ref. [10] as types that vanish in the absence of flavor structure. All three contain a Lorentz singlet pair of quarks in the antisymmetric $\overline{3}$ representation of $\mathrm{SU}(3)_{c}$, yielding $n_{g}^{3}\left(n_{g}-\right.$ 1) independent operators. The operators of type $Q_{l q^{3} H^{2}}$ are different from these three (and others identified by [10]) in that there is at least one Lagrangian term in the absence of flavor. However not all of the terms are present in the absence of flavor structure. Dimension-8 is the lowest mass dimension where this happens. The vanishing of operators in the absence of flavor structure first occurs at dimension- 7 .

19. $\psi^{4} X$.

For a pair of currents there are 114 terms formed an operator by contracting the currents with a field strength, and inserting $\mathrm{SU}(2)_{w}$ and $\mathrm{SU}(3)_{c}$ generators and invariants as necessary. There are at least two terms per $J J X$ operator type, one from $X_{L}$ and one from $X_{R}$. The largest number of terms is eight, which occurs for operator types $Q_{u^{2} d^{2} G}, Q_{q^{2} u^{2} G}$, and $Q_{q^{2} d^{2} G}$, where the $\mathrm{SU}(3)_{c}$ combinations are $(8 \otimes 1 \otimes 8),(1 \otimes 8 \otimes 8)$, $(8 \otimes 8 \otimes 8)_{A}$, and $(8 \otimes 8 \otimes 8)_{S}$ for the, say, $u$ current, $d$ current and $G$, respectively.

There are five types of operators that were identified in [10] involving identical currents contracted with the hypercharge field strength, e.g. $\left(\bar{l} \gamma^{\mu} l\right)\left(\bar{l} \gamma^{\nu} l\right) B_{\mu \nu}$, which 
vanish in the absence of flavor structure as the contraction forces the fermions into an antisymmetric flavor representation. For each Lagrangian term the number of operators is

$$
(\bar{\square} \otimes \square \otimes \bar{\square} \otimes \square)_{A}=\operatorname{adj} \oplus \operatorname{adj} \oplus \bar{a} s \oplus \bar{s} a=\frac{1}{2} n_{g}^{2}\left(n_{g}^{2}-1\right)
$$

The relevant group theory results can be found in e.g. [27]. The electron is a special case as it does not have $\mathrm{SU}(2)_{w}$ or $\mathrm{SU}(3)_{c}$ indices. As such only half of the operators of this class 19 type are independent with the rest being related through a Fierz identity.

For operators with fermion chirality $(\bar{L} R)(\bar{R} L)$ there are two possibilities per field strength, one with the left-handed field strength and the other with its right-handed counterpart. For operators with fermion chirality $(\bar{L} R)(\bar{L} R)$ there are instead three choices for the Lorentz contractions, all with $X_{R}$. Two of these terms involve a tensor bilinear and a scalar bilinear while the third has two tensor bilinears. Additionally when all the fermions are quarks there are two choices for the $\mathrm{SU}(3)_{c}$ contractions.

For the baryon number violating operators with two left-handed and two righthanded fermions there are two possible Lorentz contractions, one with $X_{L}$ and one with $X_{R}$. When these operators involve a gluon field strength there are also two possible arrangements of the $\mathrm{SU}(3)_{c}$ indices, $8 \otimes 3 \otimes 3 \otimes 3=(3 \oplus \overline{6} \ldots) \otimes 3 \otimes 3=1 \oplus 1 \ldots$ The operators of type $Q_{e q^{2} u X}$ with $X=W_{R}$ or $B_{L}$ are in antisymmetric flavor representations for the $q$ pair, and as such vanish in the absence of flavor structure. Instead $Q_{e q^{2} u X}$ with $X=W_{L}$ or $B_{R}$ are symmetric in $p$ and $r$. The types involving gluons have full flavor rank as the $q$ fields can either be a color $\overline{3}$ or 6 , compensating for other (anti)symmetries of the operator.

On the other hand, when the fermions all have the same chirality three Lorentz contractions are possible. For operators of the type $Q_{e u^{2} d B}$ the gauge contractions are fixed and it is the Lorentz contractions that dictate the flavor representation of the $u$ pair is, leading to one symmetric and one full rank term +h.c.. Instead for $Q_{e u^{2} d G}$ type operators, the additional freedom coming from the color indices of the gluon, allowing for full flavor rank, $n_{g}^{4}$, in all the Lagrangian terms. In the case of $Q_{l q^{3} X}$ only two of the three Lorentz contractions are independent due to the identical $q$ fields. There are two terms of $Q_{l q^{3} G}$ operators (+h.c.) that have the same flavor representations as the their dimension-6 analog, $Q_{q q q l}$, along with one term of $Q_{l q^{3} W}$ and one term of $Q_{l q^{3} B}$, again + h.c.. For each of these types of operators there are an equal number of operator types that have mixed symmetry, $\frac{1}{3} n_{g}^{2}\left(n_{g}^{2}-1\right)$, that vanish in the absence of flavor structure similar to $Q_{l q^{3} H^{2}}^{(3)}$. Finally there is a third term + h.c. for the operators involving $W_{\mu \nu}^{I}, Q_{l q^{3} W}^{(2)}$, that is in a symmetric plus mixed flavor representation.

Operators of the type $Q_{l q^{3} W}$ are another complicated case that deserve further discussion. Here the quarks can be in either the 2 or the 4 representation of both $\mathrm{SU}(2)_{w}$ and the $\mathrm{SU}(2)_{L}$ of the Lorentz group. Naively there are four terms (+h.c.) to consider with redundant operators handled in a similar fashion as the $Q_{l q^{3} H^{2}}$ case. However we can unambiguously combine the two terms that are Lorentz quartets 
into a single term, reducing the number of terms to three +h.c.. To see this consider the quark flavor symmetries of the four cases. Following ref. [23], specifically its table 2, we decompose the product of the gauge and Lorentz representations into irreducible representations of the permutation group of three objects, $S_{3}$, which gives us the flavor representations of the three quarks. As we have been seen before, when the quarks are in the 2 of both $\mathrm{SU}(2)_{w}$ and $\mathrm{SU}(2)_{L}$ they have symmetric, mixed, and antisymmetric flavor representations. When one of the two representations is a 2 and the other is a 4 there is only a mixed flavor representation. Finally, when both representations are the 4 there is only the symmetric representation. We can combine the two terms that are in the 4 of $\mathrm{SU}(2)_{L}$ into $Q_{l q^{3} W}^{(2)}$ as they contain distinct flavor representations. This is not unambiguously possible for $Q_{l q^{3} W}^{(1)}$ and $Q_{l q^{3} W}^{(3)}$, or $Q_{l q^{3} H^{2}}^{(2)}$ and $Q_{l q^{3} H^{2}}^{(3)}$ as each of those terms contain a mixed representation. In equations, the naive terms that are in the 4 of $\mathrm{SU}(2)_{L}$ are

$$
\begin{aligned}
& Q_{l q^{3} W}^{(2 a)}=\epsilon_{\alpha \beta \gamma}\left(\tau^{I} \epsilon\right)_{m n} \epsilon_{j k}\left(q_{p}^{m \alpha} C \sigma^{\mu \nu} q_{r}^{j \beta}\right)\left(q_{s}^{k \gamma} C l_{t}^{n}\right) W_{\mu \nu}^{I}, \\
& Q_{l q^{3} W}^{(2 b)}=\epsilon_{\alpha \beta \gamma} \epsilon_{m n}\left(\tau^{I} \epsilon\right)_{j k}\left(q_{p}^{m \alpha} C \sigma^{\mu \nu} q_{r}^{j \beta}\right)\left(q_{s}^{k \gamma} C l_{t}^{n}\right) W_{\mu \nu}^{I} .
\end{aligned}
$$

They can be combined as

$$
\left.2 Q_{p r s t}^{(2)}=\underset{p r s t}{\left(Q_{l q^{3} W}^{(2 a)}\right.}-\underset{r p s t}{Q_{l q^{3} W}^{(2 a)}}\right)-\left(\underset{p r s t}{Q_{q^{3} W}^{(2 b)}}-\underset{r p s t}{Q_{l q^{3} W}^{(2 b)}}\right)
$$

Other combinations are possible of course, but this combination makes it clear there is a symmetric and a mixed flavor representation.

20. $\psi^{4} H D$.

In class 20 the operators either have one fermion transforming as $\left(\frac{1}{2}, 0\right)$ and three transforming as $\left(0, \frac{1}{2}\right)$ under the Lorentz group, or vice versa. When describing the classification of this class we assume the former case. Then, from (3.8) the derivative cannot act on the left-handed fermion. Otherwise the operator would be reduced by the EOM.

We start with the baryon number conserving operators. Consider the case when the four fermion fields are distinguishable, e.g. $\bar{d}, d, \bar{l}, e$ (with conjugate fields are counted separately). Here there are two independent Lorentz contractions when the derivative acts on the Higgs field. A third Lorentz structure is related to the first two by eq. (3.2). When the derivative acts on a fermion there is instead only one possible Lorentz contraction. We use type $Q_{l e d^{2} H D}$ as an example. It has the five 
aforementioned candidate terms

$$
\begin{aligned}
& x_{1}=\bar{d}_{a} d_{\dot{a}} \bar{l}_{\dot{b}} e_{\dot{c}}(D H)_{b \dot{d}} \epsilon^{a b} \epsilon^{\dot{a} \dot{b}} \epsilon^{\dot{c} \dot{d}}, \\
& x_{2}=\bar{d}_{a} d_{\dot{a}} \bar{l}_{\dot{b}} e_{\dot{c}}(D H)_{b \dot{d}} \epsilon^{a b} \epsilon^{\dot{a} \dot{d}} \epsilon^{\dot{c} \dot{b}}, \\
& x_{3}=\bar{d}_{a}(D d)_{b(\dot{a} \dot{d})} \bar{l}_{\dot{b}} e_{\dot{c}} H \frac{1}{2} \epsilon^{a b}\left(2 \epsilon^{\dot{a} \dot{b}} \epsilon^{\dot{c} \dot{d}}-\epsilon^{\dot{a} \dot{d}} \epsilon^{\dot{c} \dot{b}}\right), \\
& x_{4}=\bar{d}_{a} d_{\dot{a}}(D \bar{l})_{a(\dot{b} \dot{d})} e_{\dot{c}} H \frac{1}{2} \epsilon^{a b}\left(\epsilon^{\dot{a} \dot{b}} \epsilon^{\dot{c} \dot{d}}+\epsilon^{\dot{a} \dot{d}} \epsilon^{\dot{c} \dot{b}}\right), \\
& x_{5}=\bar{d}_{a} d_{\dot{a}} l_{\dot{b}}(D e)_{a(\dot{c} \dot{d})} H \frac{1}{2} \epsilon^{a b} \epsilon^{\dot{a} \dot{b}} \epsilon^{\dot{c} \dot{d}},
\end{aligned}
$$

where eq. (3.2) is used to remove redundant Lorentz structures. There are two constraint equations

$$
\begin{aligned}
& D y_{1}=x_{1}+x_{3}+\frac{1}{2} x_{4}+\frac{1}{2} x_{5}=0 \\
& D y_{2}=x_{2}-x_{3}+\frac{1}{2} x_{4}=0
\end{aligned}
$$

and we choose to keep $x_{1}, x_{2}$, and $x_{4}$ in our basis. For other types of operators where all four fermions are distinguishable we keep the analogs of $x_{1}, x_{2}$, and $x_{4}$ as well. In addition, if there are four fields in the operator that are fundamentals under $\mathrm{SU}(2)_{w}$ or $\mathrm{SU}(3)_{c}$, including the Higgs, then there are two possible contractions of those gauge indices for each possible Lorentz contraction.

The other possibility is that only three of the fermion fields are unique, e.g. $\bar{d}, d, \bar{q}, d$. In this case there is only one way to contract the Lorentz indices when the derivative acts on the Higgs field, and only two possible ways to assign the derivative to fermions. When the derivative acts on the Higgs field or the repeated fermion there are four, two, and one possible gauge contractions when the repeated fermion is $q$, one of $\{l, u, d\}$, or $e$, respectively. Instead when the derivative acts on the fermion is not repeated, e.g. $\bar{d}, d,(D \bar{q}), d$, there are two possible gauge contractions if the repeated fermion is $q$ and only one otherwise. Here the repeated fermion is in a symmetric Lorentz representation and so the gauge contractions must be antisymmetric, eliminating half the possibilities.

The baryon number violating operators follow the same rules. There are a couple of non-trivial flavor cases. When there are duplicate right-handed fermions they form a symmetric flavor representation if the derivative acts on the Higgs field, and have full flavor rank if the derivative acts on the one of the duplicate fermions. For the term $Q_{e q^{3} H D}$, the derivative acts on one of the $q$ fields, breaking the flavor constrain, eq. (3.18), giving it full flavor rank.

21. $\psi^{4} D^{2}$.

We start with operators with equal numbers of left- and right-handed fermions, both $B$ and $\not B$. There are two ways to assign the derivatives to the fields that are not related by IBP. The first is to assign the derivatives to fermions that have the 
same Lorentz representation, and second is to assign the derivatives to fermions with conjugate Lorentz representations. When both currents are electron one of the representations vanishes in the absence of flavor structure. As previously mentioned this is due to the fact that the electron is a singlet both $\mathrm{SU}(2)_{w}$ and $\mathrm{SU}(3)_{c}$. As this work was being completed ref. [24] appeared, which classified nine terms from class 19. Some of their operators subsume both derivatives into the d'Alembertian operator. Our logic is consistent with the results of ref. [24]. The difference is we use relations like (3.8) to reduce operators with two derivatives acting on the same fermion to classes with fewer derivatives. As such the $\psi^{4} D^{2}$ operators in our basis where derivatives act symmetrically take the form $D_{\mu}\left(\psi_{1} \Gamma \psi_{2}\right) D^{\mu}\left(\psi_{3} \Gamma \psi_{4}\right)$ where $\Gamma$ is some, possibly scalar, combination of gamma matrices. The remaining 30 current-current terms and the four terms with chirality $(\bar{L} R)(\bar{R} L)$ in class 19 have a form analogous to the operators classified by ref. [24]. Also, the derivatives in $Q_{e q^{2} u D^{2}}$ break the flavor constraint present in its dimension-6 analog $Q_{q q u e}$.

For operators with either all left-handed or all right-handed fermions there are three possible Lorentz contractions, two where the fermions without derivatives form a scalar and a third where they form a tensor. An example of an IBP constraint equation for these types of operators is

$$
Q_{l e q u D^{2}}^{(1)}+2\left(D_{\mu} \bar{l}_{p}^{j} D^{\mu} e_{r}\right) \epsilon_{j k}\left(\bar{q}_{s}^{k} u_{t}\right)=E .
$$

There is a single term (+h.c.) for the type $Q_{l q^{3} D^{2}}$. As was the case with $Q_{e q^{2} u D^{2}}$, the derivatives in $Q_{l q^{3} D^{2}}$ break the flavor constraint present in some other operators of type $Q_{l q^{3} \ldots}$, allowing the term to have full flavor rank. On the other hand, for the first time we encounter a term of the type $Q_{e u^{2} d \ldots}$ that vanishes in the absence of flavor structure. There is also a second type of $Q_{e u^{2} d D^{2}}$ operator (+h.c.) that has full flavor rank.

\section{The complete set of dimension- 8 operators}

Having gone through our classification of the dimension- 8 operators in the previous section we are now ready to tabulate the results. Table 1 summarizes the results tables that follow it. The links in the rightmost column point to the table(s) of results for a given class. The number of types of operators and the number of Lagrangian terms in the class are given in the third and fourth columns from the left, respectively. For comparison the number of operators from ref. [10] is given in the second column from the right. Lines separate the classes based on the number of fermions in the class. Four-fermion operators are further divided into subclasses either preserving or violating baryon number. Additionally the number to the right of the $+\operatorname{sign}$ in $N_{\text {type,term }}$ for the four-fermion operators is the number of types or terms that vanish in the absence of flavor structure tables 2 and 3 contain bosonic operators. Tables 4, 5, 6, 7, 8, and 9 contain two-fermion operators. Tables 10, 11, 12, 13, 14, 15, 16, 17, and 18 contain four-fermions operators. 


\begin{tabular}{|c|c|c|c|c|c|}
\hline$\#$ & Class & $N_{\text {type }}$ & $N_{\text {term }}$ & $N_{\mathrm{op}}[10]$ & Table(s) \\
\hline 1 & $X^{4}$ & 7 & 43 & 43 & 2 \\
\hline 2 & $H^{8}$ & 1 & 1 & 1 & 2 \\
\hline 3 & $H^{6} D^{2}$ & 1 & 2 & 2 & 2 \\
\hline 4 & $H^{4} D^{4}$ & 1 & 3 & 3 & 2 \\
\hline 5 & $X^{3} H^{2}$ & 3 & 6 & 6 & 3 \\
\hline 6 & $X^{2} H^{4}$ & 5 & 10 & 10 & 3 \\
\hline 7 & $X^{2} H^{2} D^{2}$ & 4 & 18 & 18 & 3 \\
\hline 8 & $X H^{4} D^{2}$ & 2 & 6 & 6 & 3 \\
\hline 9 & $\psi^{2} X^{2} H$ & 16 & 96 & $96 n_{g}^{2}$ & 4 \\
\hline 10 & $\psi^{2} X H^{3}$ & 8 & 22 & $22 n_{g}^{2}$ & 5 \\
\hline 11 & $\psi^{2} H^{2} D^{3}$ & 6 & 16 & $16 n_{g}^{2}$ & 5 \\
\hline 12 & $\psi^{2} H^{5}$ & 3 & 6 & $6 n_{g}^{2}$ & 5 \\
\hline 13 & $\psi^{2} H^{4} D$ & 6 & 13 & $13 n_{g}^{2}$ & 5 \\
\hline 14 & $\psi^{2} X^{2} D$ & 21 & 57 & $57 n_{g}^{2}$ & 6,7 \\
\hline 15 & $\psi^{2} X H^{2} D$ & 16 & 92 & $92 n_{g}^{2}$ & 7,8 \\
\hline 16 & $\psi^{2} X H D^{2}$ & 8 & 48 & $48 n_{g}^{2}$ & 9 \\
\hline 17 & $\psi^{2} H^{3} D^{2}$ & 3 & 36 & $36 n_{g}^{2}$ & 9 \\
\hline $18(B)$ & \multirow{2}{*}{$\psi^{4} H^{2}$} & 19 & 75 & $n_{g}^{2}\left(67 n_{g}^{2}+n_{g}+7\right)$ & 10,11 \\
\hline $18(\not B)$ & & $4+3$ & $12+8$ & $\frac{1}{3} n_{g}^{2}\left(43 n_{g}^{2}-9 n_{g}+2\right)$ & 10 \\
\hline $19(B)$ & \multirow{2}{*}{$\psi^{4} X$} & $40+5$ & $156+12$ & $4 n_{g}^{2}\left(40 n_{g}^{2}-1\right)$ & $12,13,14$ \\
\hline $19(\not B)$ & & 4 & $44+12$ & $2 n_{g}^{3}\left(21 n_{g}+1\right)$ & 15 \\
\hline $20(B)$ & \multirow{2}{*}{$\psi^{4} H D$} & 16 & $134+2$ & $n_{g}^{3}\left(135 n_{g}-1\right)$ & 16,17 \\
\hline $20(\not B)$ & & 7 & 32 & $n_{g}^{3}\left(29 n_{g}+3\right)$ & 17 \\
\hline $21(B)$ & \multirow{2}{*}{$\psi^{4} D^{2}$} & 18 & 55 & $\frac{11}{2} n_{g}^{2}\left(9 n_{g}^{2}+1\right)$ & 10,18 \\
\hline \multirow[t]{4}{*}{$21(\not B)$} & & 4 & $10+2$ & $n_{g}^{3}\left(11 n_{g}-1\right)$ & 10 \\
\hline & $B$ & $204+5$ & $895+14$ & $895(36971), n_{g}=1(3)$ & \\
\hline & $\not B$ & $19+3$ & $98+22$ & $98(7836), n_{g}=1(3)$ & \\
\hline & Total & $223+8$ & $993+36$ & $993(44807), n_{g}=1(3)$ & \\
\hline
\end{tabular}

Table 1. Summary of the contents of the tables to follow. The links in the rightmost column point to the table(s) of results for a given class. The number of types of operators and the number of Lagrangian terms in the class are given in the third and fourth columns from the left, respectively. For comparison the number of operators from ref. [10] is given in the second column from the right. Lines separate the classes based on the number of fermions in the class. Four-fermion operators are further divided into subclasses either preserving or violating baryon number. Additionally the number to the right of the + sign in $N_{\text {type(term) }}$ for the four-fermion operators is the number of types(terms) that vanish in the absence of flavor structure. 


\begin{tabular}{|c|c|}
\hline & $1: X^{4}, X^{3} X^{\prime}$ \\
\hline$Q_{G^{4}}^{(1)}$ & $\left(G_{\mu \nu}^{A} G^{A \mu \nu}\right)\left(G_{\rho \sigma}^{B} G^{B \rho \sigma}\right)$ \\
\hline$Q_{G^{4}}^{(2)}$ & $\left(G_{\mu \nu}^{A} \widetilde{G}^{A \mu \nu}\right)\left(G_{\rho \sigma}^{B} \widetilde{G}^{B \rho \sigma}\right)$ \\
\hline$Q_{G^{4}}^{(3)}$ & $\left(G_{\mu \nu}^{A} G^{B \mu \nu}\right)\left(G_{\rho \sigma}^{A} G^{B \rho \sigma}\right)$ \\
\hline$Q_{G^{4}}^{(4)}$ & $\left(G_{\mu \nu}^{A} \widetilde{G}^{B \mu \nu}\right)\left(G_{\rho \sigma}^{A} \widetilde{G}^{B \rho \sigma}\right)$ \\
\hline$Q_{G^{4}}^{(5)}$ & $\left(G_{\mu \nu}^{A} G^{A \mu \nu}\right)\left(G_{\rho \sigma}^{B} \widetilde{G}^{B \rho \sigma}\right)$ \\
\hline$Q_{G^{4}}^{(6)}$ & $\left(G_{\mu \nu}^{A} G^{B \mu \nu}\right)\left(G_{\rho \sigma}^{A} \widetilde{G}^{B \rho \sigma}\right)$ \\
\hline$Q_{G^{4}}^{(7)}$ & $d^{A B E} d^{C D E}\left(G_{\mu \nu}^{A} G^{B \mu \nu}\right)\left(G_{\rho \sigma}^{C} G^{D \rho \sigma}\right)$ \\
\hline$Q_{G^{4}}^{(8)}$ & $d^{A B E} d^{C D E}\left(G_{\mu \nu}^{A} \widetilde{G}^{B \mu \nu}\right)\left(G_{\rho \sigma}^{C} \widetilde{G}^{D \rho \sigma}\right)$ \\
\hline$Q_{G^{4}}^{(9)}$ & $d^{A B E} d^{C D E}\left(G_{\mu \nu}^{A} G^{B \mu \nu}\right)\left(G_{\rho \sigma}^{C} \widetilde{G}^{D \rho \sigma}\right)$ \\
\hline$Q_{W^{4}}^{(1)}$ & $\left(W_{\mu \nu}^{I} W^{I \mu \nu}\right)\left(W_{\rho \sigma}^{J} W^{J \rho \sigma}\right)$ \\
\hline$Q_{W^{4}}^{(2)}$ & $\left(W_{\mu \nu}^{I} \widetilde{W}^{I \mu \nu}\right)\left(W_{\rho \sigma}^{J} \widetilde{W}^{J \rho \sigma}\right)$ \\
\hline$Q_{W^{4}}^{(3)}$ & $\left(W_{\mu \nu}^{I} W^{J \mu \nu}\right)\left(W_{\rho \sigma}^{I} W^{J \rho \sigma}\right)$ \\
\hline$Q_{W^{4}}^{(4)}$ & $\left(W_{\mu \nu}^{I} \widetilde{W}^{J \mu \nu}\right)\left(W_{\rho \sigma}^{I} \widetilde{W}^{J \rho \sigma}\right)$ \\
\hline$Q_{W^{4}}^{(5)}$ & $\left(W_{\mu \nu}^{I} W^{I \mu \nu}\right)\left(W_{\rho \sigma}^{J} \widetilde{W}^{J \rho \sigma}\right)$ \\
\hline$Q_{W^{4}}^{(6)}$ & $\left(W_{\mu \nu}^{I} W^{J \mu \nu}\right)\left(W_{\rho \sigma}^{I} \widetilde{W}^{J \rho \sigma}\right)$ \\
\hline$Q_{B^{4}}^{(1)}$ & $\left(B_{\mu \nu} B^{\mu \nu}\right)\left(B_{\rho \sigma} B^{\rho \sigma}\right)$ \\
\hline$Q_{B^{4}}^{(2)}$ & $\left(B_{\mu \nu} \widetilde{B}^{\mu \nu}\right)\left(B_{\rho \sigma} \widetilde{B}^{\rho \sigma}\right)$ \\
\hline$Q_{B^{4}}^{(3)}$ & $\left(B_{\mu \nu} B^{\mu \nu}\right)\left(B_{\rho \sigma} \widetilde{B}^{\rho \sigma}\right)$ \\
\hline$Q_{G^{3} B}^{(1)}$ & $d^{A B C}\left(B_{\mu \nu} G^{A \mu \nu}\right)\left(G_{\rho \sigma}^{B} G^{C \rho \sigma}\right)$ \\
\hline$Q_{G^{3} B}^{(2)}$ & $d^{A B C}\left(B_{\mu \nu} \widetilde{G}^{A \mu \nu}\right)\left(G_{\rho \sigma}^{B} \widetilde{G}^{C \rho \sigma}\right)$ \\
\hline$Q_{G^{3} B}^{(3)}$ & $d^{A B C}\left(B_{\mu \nu} \widetilde{G}^{A \mu \nu}\right)\left(G_{\rho \sigma}^{B} G^{C \rho \sigma}\right)$ \\
\hline$Q_{G^{3} B}^{(4)}$ & $d^{A B C}\left(B_{\mu \nu} G^{A \mu \nu}\right)\left(G_{\rho \sigma}^{B} \widetilde{G}^{C \rho \sigma}\right)$ \\
\hline
\end{tabular}

\begin{tabular}{|l|l|}
\hline \multicolumn{1}{|c|}{$\mathbf{1}: \boldsymbol{X}^{2} \boldsymbol{X}^{\prime 2}$} \\
\hline$Q_{G^{2} W^{2}}^{(1)}$ & $\left(W_{\mu \nu}^{I} W^{I \mu \nu}\right)\left(G_{\rho \sigma}^{A} G^{A \rho \sigma}\right)$ \\
$Q_{G^{2} W^{2}}^{(2)}$ & $\left(W_{\mu \nu}^{I} \widetilde{W}^{I \mu \nu}\right)\left(G_{\rho \sigma}^{A} \widetilde{G}^{A \rho \sigma}\right)$ \\
$Q_{G^{2} W^{2}}^{(3)}$ & $\left(W_{\mu \nu}^{I} G^{A \mu \nu}\right)\left(W_{\rho \sigma}^{I} G^{A \rho \sigma}\right)$ \\
$Q_{G^{2} W^{2}}^{(4)}$ & $\left(W_{\mu \nu}^{I} \widetilde{G}^{A \mu \nu}\right)\left(W_{\rho \sigma}^{I} \widetilde{G}^{A \rho \sigma}\right)$ \\
$Q_{G^{2} W^{2}}^{(5)}$ & $\left(W_{\mu \nu}^{I} \widetilde{W}^{I \mu \nu}\right)\left(G_{\rho \sigma}^{A} G^{A \rho \sigma}\right)$ \\
$Q_{G^{2} W^{2}}^{(6)}$ & $\left(W_{\mu \nu}^{I} W^{I \mu \nu}\right)\left(G_{\rho \sigma}^{A} \widetilde{G}^{A \rho \sigma}\right)$ \\
$Q_{G^{2} W^{2}}^{(7)}$ & $\left(W_{\mu \nu}^{I} G^{A \mu \nu}\right)\left(W_{\rho \sigma}^{I} \widetilde{G}^{A \rho \sigma}\right)$ \\
$Q_{G^{2} B^{2}}^{(1)}$ & $\left(B_{\mu \nu} B^{\mu \nu}\right)\left(G_{\rho \sigma}^{A} G^{A \rho \sigma}\right)$ \\
$Q_{G^{2} B^{2}}^{(2)}$ & $\left(B_{\mu \nu} \widetilde{B}^{\mu \nu}\right)\left(G_{\rho \sigma}^{A} \widetilde{G}^{A \rho \sigma}\right)$ \\
$Q_{G^{2} B^{2}}^{(3)}$ & $\left(B_{\mu \nu} G^{A \mu \nu}\right)\left(B_{\rho \sigma} G^{A \rho \sigma}\right)$ \\
$Q_{G^{2} B^{2}}^{(4)}$ & $\left(B_{\mu \nu} \widetilde{G}^{A \mu \nu}\right)\left(B_{\rho \sigma} \widetilde{G}^{A \rho \sigma}\right)$ \\
$Q_{G^{2} B^{2}}^{(5)}$ & $\left(B_{\mu \nu} \widetilde{B}^{\mu \nu}\right)\left(G_{\rho \sigma}^{A} G^{A \rho \sigma}\right)$ \\
$Q_{G^{2} B^{2}}^{(6)}$ & $\left(B_{\mu \nu} B^{\mu \nu}\right)\left(G_{\rho \sigma}^{A} \widetilde{G}^{A \rho \sigma}\right)$ \\
$Q_{G^{2} B^{2}}^{(7)}$ & $\left(B_{\mu \nu} G^{A \mu \nu}\right)\left(B_{\rho \sigma} \widetilde{G}^{A \rho \sigma}\right)$ \\
$Q_{W^{2} B^{2}}^{(1)}$ & $\left(B_{\mu \nu} B^{\mu \nu}\right)\left(W_{\rho \sigma}^{I} W^{I \rho \sigma}\right)$ \\
$Q_{W^{2} B^{2}}^{(2)}$ & $\left(B_{\mu \nu} \widetilde{B}^{\mu \nu}\right)\left(W_{\rho \sigma}^{I} \widetilde{W}^{I \rho \sigma}\right)$ \\
$Q_{W^{2} B^{2}}^{(3)}$ & $\left(B_{\mu \nu} W^{I \mu \nu}\right)\left(B_{\rho \sigma} W^{I \rho \sigma}\right)$ \\
$Q_{W^{2} B^{2}}^{(4)}$ & $\left(B_{\mu \nu} \widetilde{W}^{I \mu \nu}\right)\left(B_{\rho \sigma} \widetilde{W}^{I \rho \sigma}\right)$ \\
$Q_{W^{2} B^{2}}^{(5)}$ & $\left(B_{\mu \nu} \widetilde{B}^{\mu \nu}\right)\left(W_{\rho \sigma}^{I} W^{I \rho \sigma}\right)$ \\
$Q_{W^{2} B^{2}}^{(6)}$ & $\left(B_{\mu \nu} B^{\mu \nu}\right)\left(W_{\rho \sigma}^{I} \widetilde{W}^{I \rho \sigma}\right)$ \\
$Q^{2} B^{2}$ & $\left(B_{\mu \nu} W^{I \mu \nu}\right)\left(B_{\rho \sigma} \widetilde{W}^{I \rho \sigma}\right)$ \\
\hline
\end{tabular}

\begin{tabular}{|c|c|c|c|c|c|}
\hline \multicolumn{2}{|c|}{$2: H^{8}$} & \multicolumn{2}{|r|}{$3: H^{6} D^{2}$} & \multicolumn{2}{|r|}{$4: H^{4} D^{4}$} \\
\hline \multirow[t]{3}{*}{$Q_{H^{8}}$} & $\left(H^{\dagger} H\right)^{4}$ & $Q_{H^{6}}^{(1)}$ & $\left(H^{\dagger} H\right)^{2}\left(D_{\mu} H^{\dagger} D^{\mu} H\right)$ & $Q_{H^{4}}^{(1)}$ & $\left(D_{\mu} H^{\dagger} D_{\nu} H\right)\left(D^{\nu} H^{\dagger} D^{\mu} H\right)$ \\
\hline & & $Q_{H^{6}}^{(2)}$ & $\left(H^{\dagger} H\right)\left(H^{\dagger} \tau^{I} H\right)\left(D_{\mu} H^{\dagger} \tau^{I} D^{\mu} H\right)$ & $Q_{H^{4}}^{(2)}$ & $\left(D_{\mu} H^{\dagger} D_{\nu} H\right)\left(D^{\mu} H^{\dagger} D^{\nu} H\right)$ \\
\hline & & & & $Q_{H^{4}}^{(3)}$ & $\left(D^{\mu} H^{\dagger} D_{\mu} H\right)\left(D^{\nu} H^{\dagger} D_{\nu} H\right)$ \\
\hline
\end{tabular}

Table 2. The dimension-eight operators in the SMEFT whose field content is either entirely gauge field strengths or Higgs boson fields.

\subsection{Results for bosonic operators}

See tables 2 and 3. 


\begin{tabular}{|c|c|}
\hline \multicolumn{2}{|c|}{$\mathbf{5}: \boldsymbol{X}^{3} \boldsymbol{H}^{2}$} \\
\hline$Q_{G^{3} H^{2}}^{(1)}$ & $f^{A B C}\left(H^{\dagger} H\right) G_{\mu}^{A \nu} G_{\nu}^{B \rho} G_{\rho}^{C \mu}$ \\
$Q_{G^{3} H^{2}}^{A B C}$ & $f^{A B C}\left(H^{\dagger} H\right) G_{\mu}^{A \nu} G_{\nu}^{B \rho} \widetilde{G}_{\rho}^{C \mu}$ \\
$Q_{W}^{(1)}$ & $\epsilon^{I J K}\left(H^{\dagger} H\right) W_{\mu}^{I \nu} W_{\nu}^{J \rho} W_{\rho}^{K \mu}$ \\
$Q_{W^{3} H^{2}}^{(2)}$ & $\epsilon^{I J K}\left(H^{\dagger} H\right) W_{\mu}^{I \nu} W_{\nu}^{J \rho} \widetilde{W}_{\rho}^{K \mu}$ \\
$Q_{W}^{(1)}{ }^{2} B H^{2}$ & $\epsilon^{I J K}\left(H^{\dagger} \tau^{I} H\right) B_{\mu}^{\nu} W_{\nu}^{J \rho} W_{\rho}^{K \mu}$ \\
$Q_{W^{2} B H^{2}}^{(2)}$ & $\epsilon^{I J K}\left(H^{\dagger} \tau^{I} H\right)\left(\widetilde{B}^{\mu \nu} W_{\nu \rho}^{J} W_{\mu}^{K \rho}+B^{\mu \nu} W_{\nu \rho}^{J} \widetilde{W}_{\mu}^{K \rho}\right)$ \\
\hline
\end{tabular}

\begin{tabular}{|c|c|}
\hline \multicolumn{2}{|c|}{$\mathbf{6}: \boldsymbol{X}^{2} \boldsymbol{H}^{4}$} \\
\hline$Q_{G^{2} H^{4}}^{(1)}$ & $\left(H^{\dagger} H\right)^{2} G_{\mu \nu}^{A} G^{A \mu \nu}$ \\
$Q_{G^{2}}^{(2)} H^{4}$ & $\left(H^{\dagger} H\right)^{2} \widetilde{G}_{\mu \nu}^{A} G^{A \mu \nu}$ \\
$Q_{W}^{(1)} H^{4}$ & $\left(H^{\dagger} H\right)^{2} W_{\mu \nu}^{I} W^{I \mu \nu}$ \\
$Q_{W}^{(2)} H^{4}$ & $\left(H^{\dagger} H\right)^{2} \widetilde{W}_{\mu \nu}^{I} W^{I \mu \nu}$ \\
$Q_{W}^{(3)} H^{4}$ & $\left(H^{\dagger} \tau^{I} H\right)\left(H^{\dagger} \tau^{J} H\right) W_{\mu \nu}^{I} W^{J \mu \nu}$ \\
$Q_{W}^{(4)} H^{4}$ & $\left(H^{\dagger} \tau^{I} H\right)\left(H^{\dagger} \tau^{J} H\right) \widetilde{W}_{\mu \nu}^{I} W^{J \mu \nu}$ \\
$Q_{W}^{(1)} B H^{4}$ & $\left(H^{\dagger} H\right)\left(H^{\dagger} \tau^{I} H\right) W_{\mu \nu}^{I} B^{\mu \nu}$ \\
$Q_{W}^{(2)}$ & $\left(H^{\dagger} H\right)\left(H^{\dagger} \tau^{I} H\right) \widetilde{W}_{\mu \nu}^{I} B^{\mu \nu}$ \\
$Q_{B^{2}}^{(1)} H^{4}$ & $\left(H^{\dagger} H\right)^{2} B_{\mu \nu} B^{\mu \nu}$ \\
$Q_{B^{2} H^{4}}^{(2)}$ & $\left(H^{\dagger} H\right)^{2} \widetilde{B}_{\mu \nu} B^{\mu \nu}$ \\
\hline \multicolumn{2}{|c}{} \\
\hline
\end{tabular}

\begin{tabular}{|l|c|}
\hline & $\mathbf{7}: \boldsymbol{X}^{2} \boldsymbol{H}^{2} \boldsymbol{D}^{2}$ \\
\hline$Q_{G^{2} H^{2} D^{2}}^{(1)}$ & $\left(D^{\mu} H^{\dagger} D^{\nu} H\right) G_{\mu \rho}^{A} G_{\nu}^{A \rho}$ \\
$Q_{G^{2}}^{(2)} H^{2} D^{2}$ & $\left(D^{\mu} H^{\dagger} D_{\mu} H\right) G_{\nu \rho}^{A} G^{A \nu \rho}$ \\
$Q_{G}^{(3)} H^{2} D^{2}$ & $\left(D^{\mu} H^{\dagger} D_{\mu} H\right) G_{\nu \rho}^{A} \widetilde{G}^{A \nu \rho}$ \\
$Q_{W}^{(1)} H^{2} D^{2}$ & $\left(D^{\mu} H^{\dagger} D^{\nu} H\right) W_{\mu \rho}^{I} W_{\nu}^{I \rho}$ \\
$Q_{W}^{(2)} H^{2} D^{2}$ & $\left(D^{\mu} H^{\dagger} D_{\mu} H\right) W_{\nu \rho}^{I} W^{I \nu \rho}$ \\
$Q_{W}^{(3)} H^{2} D^{2}$ & $\left(D^{\mu} H^{\dagger} D_{\mu} H\right) W_{\nu \rho}^{I} \widetilde{W}^{I \nu \rho}$ \\
$Q_{W}^{(4)} H^{2} D^{2}$ & $i \epsilon^{I J K}\left(D^{\mu} H^{\dagger} \tau^{I} D^{\nu} H\right) W_{\mu \rho}^{J} W_{\nu}^{K \rho}$ \\
$Q_{W}^{(5)} H^{2} D^{2}$ & $\epsilon^{I J K}\left(D^{\mu} H^{\dagger} \tau^{I} D^{\nu} H\right)\left(W_{\mu \rho}^{J} \widetilde{W}_{\nu}^{K \rho}-\widetilde{W}_{\mu \rho}^{J} W_{\nu}^{K \rho}\right)$ \\
$Q_{W}^{(6)} H^{2} D^{2}$ & $i \epsilon^{I J K}\left(D^{\mu} H^{\dagger} \tau^{I} D^{\nu} H\right)\left(W_{\mu \rho}^{J} \widetilde{W}_{\nu}^{K \rho}+\widetilde{W}_{\mu \rho}^{J} W_{\nu}^{K \rho}\right)$ \\
$Q_{W B}^{(1)} H^{2} D^{2}$ & $\left(D^{\mu} H^{\dagger} \tau^{I} D_{\mu} H\right) B_{\nu \rho} W^{I \nu \rho}$ \\
$Q_{W B}^{(2)} H^{2} D^{2}$ & $\left(D^{\mu} H^{\dagger} \tau^{I} D_{\mu} H\right) B_{\nu \rho} \widetilde{W}^{I \nu \rho}$ \\
$Q_{W B}^{(3)} H^{2} D^{2}$ & $i\left(D^{\mu} H^{\dagger} \tau^{I} D^{\nu} H\right)\left(B_{\mu \rho} W_{\nu}^{I \rho}-B_{\nu \rho} W_{\mu}^{I \rho}\right)$ \\
$Q_{W B}^{(4)} H^{2} D^{2}$ & $\left(D^{\mu} H^{\dagger} \tau^{I} D^{\nu} H\right)\left(B_{\mu \rho} W_{\nu}^{I \rho}+B_{\nu \rho} W_{\mu}^{I \rho}\right)$ \\
$Q_{W B}^{(5)} H^{2} D^{2}$ & $i\left(D^{\mu} H^{\dagger} \tau^{I} D^{\nu} H\right)\left(B_{\mu \rho} \widetilde{W}_{\nu}^{I} \rho-B_{\nu \rho} \widetilde{W}_{\mu}^{I} \rho\right)$ \\
$Q_{W}^{(6)} B H^{2} D^{2}$ & $\left(D^{\mu} H^{\dagger} \tau^{I} D^{\nu} H\right)\left(B_{\mu \rho} \widetilde{W}_{\nu}^{I} \rho+B_{\nu \rho} \widetilde{W}_{\mu}^{I} \rho\right)$ \\
$Q_{B}^{(1)} H^{2} D^{2}$ & $\left(D^{\mu} H^{\dagger} D^{\nu} H\right) B_{\mu \rho} B_{\nu}{ }^{\rho}$ \\
$Q_{B}^{(2)} H^{2} D^{2}$ & $\left(D^{\mu} H^{\dagger} D_{\mu} H\right) B_{\nu \rho} B^{\nu \rho}$ \\
$Q_{B^{2} H^{2} D^{2}}^{(3)}$ & $\left(D^{\mu} H^{\dagger} D_{\mu} H\right) B_{\nu \rho} \widetilde{B}^{\nu \rho}$ \\
\hline
\end{tabular}

\begin{tabular}{|l|c|}
\hline \multicolumn{2}{|c|}{$\mathbf{8}: \boldsymbol{X} \boldsymbol{H}^{4} \boldsymbol{D}^{2}$} \\
\hline$Q_{W H^{4} D^{2}}^{(1)}$ & $\left(H^{\dagger} H\right)\left(D^{\mu} H^{\dagger} \tau^{I} D^{\nu} H\right) W_{\mu \nu}^{I}$ \\
$Q_{W}^{(2)} H^{4} D^{2}$ & $\left(H^{\dagger} H\right)\left(D^{\mu} H^{\dagger} \tau^{I} D^{\nu} H\right) W_{\mu \nu}^{I}$ \\
$Q_{W}^{(3)} H^{4} D^{2}$ & $\epsilon^{I J K}\left(H^{\dagger} \tau^{I} H\right)\left(D^{\mu} H^{\dagger} \tau^{J} D^{\nu} H\right) W_{\mu \nu}^{K}$ \\
$Q_{W}^{(4)} H^{4} D^{2}$ & $\epsilon^{I J K}\left(H^{\dagger} \tau^{I} H\right)\left(D^{\mu} H^{\dagger} \tau^{J} D^{\nu} H\right) \widetilde{W}_{\mu \nu}^{K}$ \\
$Q_{B H^{4} D^{2}}^{(1)}$ & $\left(H^{\dagger} H\right)\left(D^{\mu} H^{\dagger} D^{\nu} H\right) B_{\mu \nu}$ \\
$Q_{B H^{4} D^{2}}^{(2)}$ & $\left(H^{\dagger} H\right)\left(D^{\mu} H^{\dagger} D^{\nu} H\right) \widetilde{B}_{\mu \nu}$ \\
\hline
\end{tabular}

Table 3. Bosonic dimension-eight operators in the SMEFT containing both gauge field strengths and Higgs boson fields.

\subsection{Results for two-fermion operators}

See tables 4, 5, 6, 7, 8 and 9 .

\subsection{Results for four-fermion operators}

See tables 10, 11, 12, 13, 14, 15, 16, 17 and 18.

\section{Phenomenology}

In this section we briefly discuss a few aspects of phenomenology involving dimension- 8 operators, focusing on processes that first start at dimension- 8 and/or involve interplay between dimension- 6 and -8 effects. In particular, we discuss light-by-light scattering and electroweak precision data. We also present a model where dimension- 8 effects are arguably more important than dimension- 6 effects. 


\begin{tabular}{|c|c|}
\hline & $\mathbf{9}: \boldsymbol{\psi}^{2} \boldsymbol{X}^{2} \boldsymbol{H}+\mathbf{h . c .}$ \\
\hline$Q_{l e G^{2} H}^{(1)}$ & $\left(\bar{l}_{p} e_{r}\right) H G_{\mu \nu}^{A} G^{A \mu \nu}$ \\
$Q_{l e G^{2} H}^{(2)}$ & $\left(\bar{l}_{p} e_{r}\right) H \widetilde{G}_{\mu \nu}^{A} G^{A \mu \nu}$ \\
$Q_{l e W^{2} H}^{(1)}$ & $\left(\bar{l}_{p} e_{r}\right) H W_{\mu \nu}^{I} W^{I \mu \nu}$ \\
$Q_{l e W^{2} H}^{(2)}$ & $\left(\bar{l}_{p} e_{r}\right) H \widetilde{W}_{\mu \nu}^{I} W^{I \mu \nu}$ \\
$Q_{l e W^{2} H}^{(3)}$ & $\epsilon^{I J K}\left(\bar{l}_{p} \sigma^{\mu \nu} e_{r}\right) \tau^{I} H W_{\mu \rho}^{J} W_{\nu}^{K \rho}$ \\
$Q_{q u G^{2} H}^{(1)}$ & $\left(\bar{q}_{p} u_{r}\right) \widetilde{H} G_{\mu \nu}^{A} G^{A \mu \nu}$ \\
$Q_{q u G^{2} H}^{(2)}$ & $\left(\bar{q}_{p} u_{r}\right) \widetilde{H} \widetilde{G}_{\mu \nu}^{A} G^{A \mu \nu}$ \\
$Q_{q u G^{2} H}^{(3)}$ & $d^{A B C}\left(\bar{q}_{p} T^{A} u_{r}\right) \widetilde{H} G_{\mu \nu}^{B} G^{C \mu \nu}$ \\
$Q_{q u G^{2} H}^{(4)}$ & $d^{A B C}\left(\bar{q}_{p} T^{A} u_{r}\right) \widetilde{H} \widetilde{G}_{\mu \nu}^{B} G^{C \mu \nu}$ \\
$Q_{q u G^{2} H}^{(5)}$ & $f^{A B C}\left(\bar{q}_{p} \sigma^{\mu \nu} T^{A} u_{r}\right) \widetilde{H} G_{\mu \rho}^{B} G_{\nu}^{C \rho}$ \\
$Q_{q u G W H}^{(1)}$ & $\left(\bar{q}_{p} T^{A} u_{r}\right) \tau^{I} \widetilde{H} G_{\mu \nu}^{A} W^{I \mu \nu}$ \\
$Q_{q u G W H}^{(2)}$ & $\left(\bar{q}_{p} T^{A} u_{r}\right) \tau^{I} \widetilde{H} \widetilde{G}_{\mu \nu}^{A} W^{I \mu \nu}$ \\
$Q_{q u G W H}^{(3)}$ & $\left(\bar{q}_{p} \sigma^{\mu \nu} T^{A} u_{r}\right) \tau^{I} \widetilde{H} G_{\mu \rho}^{A} W_{\nu}^{I \rho}$ \\
$Q_{q u G B H}^{(1)}$ & $\left(\bar{q}_{p} T^{A} u_{r}\right) \widetilde{H} G_{\mu \nu}^{A} B^{\mu \nu}$ \\
$Q_{q u G B H}^{(2)}$ & $\left(\bar{q}_{p} T^{A} u_{r}\right) \widetilde{H} \widetilde{G}_{\mu \nu}^{A} B^{\mu \nu}$ \\
$Q_{q u G B H}^{(3)}$ & $\left(\bar{q}_{p} \sigma^{\mu \nu} T^{A} u_{r}\right) \widetilde{H} G_{\mu \rho}^{A} B_{\nu}{ }^{\rho}$ \\
$Q_{q u W^{2} H}^{(1)}$ & $\left(\bar{q}_{p} u_{r}\right) \widetilde{H} W_{\mu \nu}^{I} W^{I \mu \nu}$ \\
$Q_{q u W^{2} H}^{(2)}$ & $\left(\bar{q}_{p} u_{r}\right) \widetilde{H} \widetilde{W}_{\mu \nu}^{I} W^{I \mu \nu}$ \\
$Q_{q u W^{2} H}^{(3)}$ & $\epsilon^{I J K}\left(\bar{q}_{p} \sigma^{\mu \nu} u_{r}\right) \tau^{I} \widetilde{H} W_{\mu \rho}^{J} W_{\nu}^{K \rho}$ \\
$Q_{q u W B H}^{(3)}$ & $\left(\bar{q}_{p} \sigma^{\mu \nu} u_{r}\right) \tau^{I} \widetilde{H} W_{\mu \rho}^{I} B_{\nu}{ }^{\rho}$ \\
$Q_{q u W B H}^{(1)}$ & $\left(\bar{q}_{p} u_{r}\right) \tau^{I} \widetilde{H} W_{\mu \nu}^{I} B^{\mu \nu}$ \\
$Q_{q u W B H}^{(2)}$ & $\left(\bar{q}_{p} u_{r}\right) \tau^{I} \widetilde{H} \widetilde{W}_{\mu \nu}^{I} B^{\mu \nu}$ \\
$Q_{q u B^{2} H}^{(1)}$ & $\left(\bar{q}_{p} u_{r}\right) \widetilde{H} B_{\mu \nu} B^{\mu \nu}$ \\
$Q_{q u B^{2} H}^{(2)}$ & $\left(\bar{q}_{p} u_{r}\right) \widetilde{H} \widetilde{B}_{\mu \nu} B^{\mu \nu}$ \\
\hline & \\
\hline
\end{tabular}

\begin{tabular}{|c|c|}
\hline & $\mathbf{9}: \boldsymbol{\psi}^{\mathbf{2}} \boldsymbol{X}^{2} \boldsymbol{H}+$ h.c. \\
\hline$Q_{l e W B H}^{(1)}$ & $\left(\bar{l}_{p} e_{r}\right) \tau^{I} H W_{\mu \nu}^{I} B^{\mu \nu}$ \\
$Q_{l e W B H}^{(2)}$ & $\left(\bar{l}_{p} e_{r}\right) \tau^{I} H \widetilde{W}_{\mu \nu}^{I} B^{\mu \nu}$ \\
$Q_{l e W B H}^{(3)}$ & $\left(\bar{l}_{p} \sigma^{\mu \nu} e_{r}\right) \tau^{I} H W_{\mu \rho}^{I} B_{\nu}{ }^{\rho}$ \\
$Q_{l e B^{2} H}^{(1)}$ & $\left(\bar{l}_{p} e_{r}\right) H B_{\mu \nu} B^{\mu \nu}$ \\
$Q_{l e B^{2} H}^{(2)}$ & $\left(\bar{l}_{p} e_{r}\right) H \widetilde{B}_{\mu \nu} B^{\mu \nu}$ \\
$Q_{q d G^{2} H}^{(1)}$ & $\left(\bar{q}_{p} d_{r}\right) H G_{\mu \nu}^{A} G^{A \mu \nu}$ \\
$Q_{q d G^{2} H}^{(2)}$ & $\left(\bar{q}_{p} d_{r}\right) H \widetilde{G}_{\mu \nu}^{A} G^{A \mu \nu}$ \\
$Q_{q d G^{2} H}^{(3)}$ & $d^{A B C}\left(\bar{q}_{p} T^{A} d_{r}\right) H G_{\mu \nu}^{B} G^{C \mu \nu}$ \\
$Q_{q d G^{2} H}^{(4)}$ & $d^{A B C}\left(\bar{q}_{p} T^{A} d_{r}\right) H \widetilde{G}_{\mu \nu}^{B} G^{C \mu \nu}$ \\
$Q_{q d G^{2} H}^{(5)}$ & $f^{A B C}\left(\bar{q}_{p} \sigma^{\mu \nu} T^{A} d_{r}\right) H G_{\mu \rho}^{B} G_{\nu}^{C \rho}$ \\
$Q_{q d G W H}^{(1)}$ & $\left(\bar{q}_{p} T^{A} d_{r}\right) \tau^{I} H G_{\mu \nu}^{A} W^{I \mu \nu}$ \\
$Q_{q d G W H}^{(2)}$ & $\left(\bar{q}_{p} T^{A} d_{r}\right) \tau^{I} H \widetilde{G}_{\mu \nu}^{A} W^{I \mu \nu}$ \\
$Q_{q d G W H}^{(3)}$ & $\left(\bar{q}_{p} \sigma^{\mu \nu} T^{A} d_{r}\right) \tau^{I} H G_{\mu \rho}^{A} W_{\nu}^{I \rho}$ \\
$Q_{q d G B H}^{(1)}$ & $\left(\bar{q}_{p} T^{A} d_{r}\right) H G_{\mu \nu}^{A} B^{\mu \nu}$ \\
$Q_{q d G B H}^{(2)}$ & $\left(\bar{q}_{p} T^{A} d_{r}\right) H \widetilde{G}_{\mu \nu}^{A} B^{\mu \nu}$ \\
$Q_{q d G B H}^{(3)}$ & $\left(\bar{q}_{p} \sigma^{\mu \nu} T^{A} d_{r}\right) H G_{\mu \rho}^{A} B_{\nu}{ }^{\rho}$ \\
$Q_{q d W^{2} H}^{(1)}$ & $\left(\bar{q}_{p} d_{r}\right) H W_{\mu \nu}^{I} W^{I \mu \nu}$ \\
$Q_{q d W W^{2} H}^{(2)}$ & $\left(\bar{q}_{p} d_{r}\right) H \widetilde{W}_{\mu \nu}^{I} W^{I \mu \nu}$ \\
$Q_{q d W^{2} H}^{(3)}$ & $\epsilon^{I J K}\left(\bar{q}_{p} \sigma^{\mu \nu} d_{r}\right) \tau^{I} H W_{\mu \rho}^{J} W_{\nu}^{K \rho}$ \\
$Q_{q d W B H}^{(1)}$ & $\left(\bar{q}_{p} d_{r}\right) \tau^{I} H W_{\mu \nu}^{I} B^{\mu \nu}$ \\
$Q_{q d W B H}^{(2)}$ & $\left(\bar{q}_{p} d_{r}\right) \tau^{I} H \widetilde{W}_{\mu \nu}^{I} B^{\mu \nu}$ \\
$Q_{q d W B H}^{(3)}$ & $\left(\bar{q}_{p} \sigma^{\mu \nu} d_{r}\right) \tau^{I} H W_{\mu \rho}^{I} B_{\nu}{ }^{\rho}$ \\
$Q_{q d B^{2} H}^{(1)}$ & $\left(\bar{q}_{p} d_{r}\right) H B_{\mu \nu} B^{\mu \nu}$ \\
$Q_{q d B^{2} H}^{(2)}$ & $\left(\bar{q}_{p} d_{r}\right) H \widetilde{B}_{\mu \nu} B^{\mu \nu}$ \\
\hline &
\end{tabular}

Table 4. The dimension-eight operators in the SMEFT of class-9 with field content $\psi^{2} X^{2} H$. All of the operators have Hermitian conjugates. The subscripts $p, r$ are weak-eigenstate indices. 


\begin{tabular}{|l|c|}
\hline \multicolumn{2}{|c|}{$\mathbf{1 0}: \boldsymbol{\psi}^{\mathbf{2}} \boldsymbol{X} \boldsymbol{H}^{\mathbf{3}}+$ h.c. } \\
\hline$Q_{l e W H^{3}}^{(1)}$ & $\left(\bar{l}_{p} \sigma^{\mu \nu} e_{r}\right) \tau^{I} H\left(H^{\dagger} H\right) W_{\mu \nu}^{I}$ \\
$Q_{l e W H^{3}}^{(2)}$ & $\left(\bar{l}_{p} \sigma^{\mu \nu} e_{r}\right) H\left(H^{\dagger} \tau^{I} H\right) W_{\mu \nu}^{I}$ \\
$Q_{l e B H^{3}}$ & $\left(\bar{l}_{p} \sigma^{\mu \nu} e_{r}\right) H\left(H^{\dagger} H\right) B_{\mu \nu}$ \\
$Q_{q u G H^{3}}$ & $\left(\bar{q}_{p} \sigma^{\mu \nu} T^{A} u_{r}\right) \widetilde{H}\left(H^{\dagger} H\right) G_{\mu \nu}^{A}$ \\
$Q_{q u W H^{3}}^{(1)}$ & $\left(\bar{q}_{p} \sigma^{\mu \nu} u_{r}\right) \tau^{I} \widetilde{H}\left(H^{\dagger} H\right) W_{\mu \nu}^{I}$ \\
$Q_{q u W H^{3}}^{(2)}$ & $\left(\bar{q}_{p} \sigma^{\mu \nu} u_{r}\right) \widetilde{H}\left(H^{\dagger} \tau^{I} H\right) W_{\mu \nu}^{I}$ \\
$Q_{q u B H^{3}}$ & $\left(\bar{q}_{p} \sigma^{\mu \nu} u_{r}\right) \tilde{H}\left(H^{\dagger} H\right) B_{\mu \nu}$ \\
$Q_{q d G H^{3}}$ & $\left(\bar{q}_{p} \sigma^{\mu \nu} T^{A} d_{r}\right) H\left(H^{\dagger} H\right) G_{\mu \nu}^{A}$ \\
$Q_{q d W H^{3}}^{(1)}$ & $\left(\bar{q}_{p} \sigma^{\mu \nu} d_{r}\right) \tau^{I} H\left(H^{\dagger} H\right) W_{\mu \nu}^{I}$ \\
$Q_{q d W H^{3}}^{(2)}$ & $\left(\bar{q}_{p} \sigma^{\mu \nu} d_{r}\right) H\left(H^{\dagger} \tau^{I} H\right) W_{\mu \nu}^{I}$ \\
$Q_{q d B H^{3}}$ & $\left(\bar{q}_{p} \sigma^{\mu \nu} d_{r}\right) H\left(H^{\dagger} H\right) B_{\mu \nu}$ \\
\hline
\end{tabular}

\begin{tabular}{|c|c|}
\hline \multicolumn{2}{|c|}{$\mathbf{1 1}: \boldsymbol{\psi}^{2} \boldsymbol{H}^{2} D^{3}$} \\
\hline$Q_{l^{2} H^{2} D^{3}}^{(1)}$ & $i\left(\bar{l}_{p} \gamma^{\mu} D^{\nu} l_{r}\right)\left(D_{(\mu} D_{\nu)} H^{\dagger} H\right)$ \\
$Q_{l^{2} H^{2} D^{3}}^{(2)}$ & $i\left(\bar{l}_{p} \gamma^{\mu} D^{\nu} l_{r}\right)\left(H^{\dagger} D_{(\mu} D_{\nu)} H\right)$ \\
$Q_{l^{2} H^{2} D^{3}}^{(3)}$ & $i\left(\bar{l}_{p} \gamma^{\mu} \tau^{I} D^{\nu} l_{r}\right)\left(D_{(\mu} D_{\nu)} H^{\dagger} \tau^{I} H\right)$ \\
$Q_{l^{2} H^{2} D^{3}}^{(4)}$ & $i\left(\bar{l}_{p} \gamma^{\mu} \tau^{I} D^{\nu} l_{r}\right)\left(H^{\dagger} \tau^{I} D_{(\mu} D_{\nu)} H\right)$ \\
$Q_{e^{2} H^{2} D^{3}}^{(1)}$ & $i\left(\bar{e}_{p} \gamma^{\mu} D^{\nu} e_{r}\right)\left(D_{(\mu} D_{\nu)} H^{\dagger} H\right)$ \\
$Q_{e^{2} H^{2} D^{3}}^{(2)}$ & $i\left(\bar{e}_{p} \gamma^{\mu} D^{\nu} e_{r}\right)\left(H^{\dagger} D_{(\mu} D_{\nu)} H\right)$ \\
$Q_{q^{2} H^{2} D^{3}}^{(1)}$ & $i\left(\bar{q}_{p} \gamma^{\mu} D^{\nu} q_{r}\right)\left(D_{(\mu} D_{\nu)} H^{\dagger} H\right)$ \\
$Q_{q^{2} H^{2} D^{3}}^{(2)}$ & $i\left(\bar{q}_{p} \gamma^{\mu} D^{\nu} q_{r}\right)\left(H^{\dagger} D_{(\mu} D_{\nu)} H\right)$ \\
$Q_{q^{2} H^{2} D^{3}}^{(3)}$ & $i\left(\bar{q}_{p} \gamma^{\mu} \tau^{I} D^{\nu} q_{r}\right)\left(D_{(\mu} D_{\nu)} H^{\dagger} \tau^{I} H\right)$ \\
$Q_{q^{2} H^{2} D^{2}}^{(4)}$ & $i\left(\bar{q}_{p} \gamma^{\mu} \tau^{I} D^{\nu} q_{r}\right)\left(H^{\dagger} \tau^{I} D_{(\mu} D_{\nu)} H\right)$ \\
$Q_{u^{2} H^{2} D^{3}}^{(1)}$ & $i\left(\bar{u}_{p} \gamma^{\mu} D^{\nu} u_{r}\right)\left(D_{(\mu} D_{\nu)} H^{\dagger} H\right)$ \\
$Q_{u^{2} H^{2} D^{3}}^{(2)}$ & $i\left(\bar{u}_{p} \gamma^{\mu} D^{\nu} u_{r}\right)\left(H^{\dagger} D_{(\mu} D_{\nu)} H\right)$ \\
$Q_{d^{2} H^{2} D^{3}}^{(1)}$ & $i\left(\bar{d}_{p} \gamma^{\mu} D^{\nu} d_{r}\right)\left(D_{(\mu} D_{\nu)} H^{\dagger} H\right)$ \\
$Q_{d^{2} H^{2} D^{3}}^{(2)}$ & $i\left(\bar{d}_{p} \gamma^{\mu} D^{\nu} d_{r}\right)\left(H^{\dagger} D_{(\mu} D_{\nu)} H\right)$ \\
$Q_{u d H^{2} D^{3}+\mathrm{h} . c .}$ & $i\left(\bar{u}_{p} \gamma^{\mu} D^{\nu} d_{r}\right)\left(\widetilde{H}^{\dagger} D_{(\mu} D_{\nu)} H\right)$ \\
\hline
\end{tabular}

\begin{tabular}{|c|c|}
\hline \multicolumn{2}{|c|}{$12: \boldsymbol{\psi}^{\mathbf{2}} \boldsymbol{H}^{\mathbf{5}}+$ h.c. } \\
\hline$Q_{l e H^{5}}$ & $\left(H^{\dagger} H\right)^{2}\left(\bar{l}_{p} e_{r} H\right)$ \\
$Q_{q u H^{5}}$ & $\left(H^{\dagger} H\right)^{2}\left(\bar{q}_{p} u_{r} \widetilde{H}\right)$ \\
$Q_{q d H^{5}}$ & $\left(H^{\dagger} H\right)^{2}\left(\bar{q}_{p} d_{r} H\right)$ \\
\hline
\end{tabular}

\begin{tabular}{|c|c|}
\hline \multicolumn{2}{|c|}{$\mathbf{1 3}: \boldsymbol{\psi}^{2} \boldsymbol{H}^{4} \boldsymbol{D}$} \\
\hline$Q_{l^{2} H^{4} D}^{(1)}$ & $i\left(l_{p} \gamma^{\mu} l_{r}\right)\left(H^{\dagger} \overleftrightarrow{D}_{\mu} H\right)\left(H^{\dagger} H\right)$ \\
$Q_{l^{2} H^{4} D}^{(2)}$ & $i\left(l_{p} \gamma^{\mu} \tau^{I} l_{r}\right)\left[\left(H^{\dagger} \overleftrightarrow{D}_{\mu}^{I} H\right)\left(H^{\dagger} H\right)+\left(H^{\dagger} \overleftrightarrow{D}_{\mu} H\right)\left(H^{\dagger} \tau^{I} H\right)\right]$ \\
$Q_{l^{2} H^{4} D}^{(3)}$ & $i \epsilon^{I J K}\left(l_{p} \gamma^{\mu} \tau^{I} l_{r}\right)\left(H^{\dagger} \overleftrightarrow{D}_{\mu}^{J} H\right)\left(H^{\dagger} \tau^{K} H\right)$ \\
$Q_{l^{2} H^{4} D}^{(4)}$ & $\epsilon^{I J K}\left(l_{p} \gamma^{\mu} \tau^{I} l_{r}\right)\left(H^{\dagger} \tau^{J} H\right) D_{\mu}\left(H^{\dagger} \tau^{K} H\right)$ \\
$Q_{e^{2} H^{4} D}^{(1)}$ & $i\left(e_{p} \gamma^{\mu} e_{r}\right)\left(H^{\dagger} \overleftrightarrow{D}_{\mu} H\right)\left(H^{\dagger} H\right)$ \\
$Q_{q^{2} H^{4} D}^{(2)}$ & $i\left(q_{p} \gamma^{\mu} q_{r}\right)\left(H^{\dagger} \overleftrightarrow{D}_{\mu} H\right)\left(H^{\dagger} H\right)$ \\
$Q_{q^{2} H^{4} D}^{(3)}$ & $i\left(q_{p} \gamma^{\mu} \tau^{I} q_{r}\right)\left[\left(H^{\dagger} \overleftrightarrow{D}_{\mu}^{I} H\right)\left(H^{\dagger} H\right)+\left(H^{\dagger} \overleftrightarrow{D}_{\mu} H\right)\left(H^{\dagger} \tau^{I} H\right)\right]$ \\
$Q_{q^{2} H^{4} D}^{(4)}$ & $i \epsilon^{I J K}\left(q_{p} \gamma^{\mu} \tau^{I} q_{r}\right)\left(H^{\dagger} \overleftrightarrow{D}_{\mu}^{J} H\right)\left(H^{\dagger} \tau^{K} H\right)$ \\
$Q_{q^{2} H^{4} D}$ & $\epsilon^{I J K}\left(q_{p} \gamma^{\mu} \tau^{I} q_{r}\right)\left(H^{\dagger} \tau^{J} H\right) D_{\mu}\left(H^{\dagger} \tau^{K} H\right)$ \\
$Q_{u^{2} H^{4} D}$ & $i\left(u_{p} \gamma^{\mu} u_{r}\right)\left(H^{\dagger} \overleftrightarrow{D}_{\mu} H\right)\left(H^{\dagger} H\right)$ \\
$Q_{d^{2} H^{4} D}$ & $i\left(d_{p} \gamma^{\mu} d_{r}\right)\left(H^{\dagger} \overleftrightarrow{D}_{\mu} H\right)\left(H^{\dagger} H\right)$ \\
$Q_{u d H^{4} D}+$ h.c. & $i\left(u_{p} \gamma^{\mu} d_{r}\right)\left(\widetilde{H}^{\dagger} \overleftrightarrow{D}_{\mu} H\right)\left(H^{\dagger} H\right)$ \\
\hline
\end{tabular}

Table 5. The dimension-eight operators in the SMEFT of classes-10, through -13, all of which have two fermions. The operators $Q_{u d H^{4} D}$ and $Q_{u d H^{2} D^{3}}$ have Hermitian conjugates. The subscripts $p, r$ are weak-eigenstate indices. 


\begin{tabular}{|c|c|}
\hline \multicolumn{2}{|c|}{$14: \psi^{2} \boldsymbol{X}^{2} \boldsymbol{D}$} \\
\hline$Q_{q^{2} G^{2} D}^{(1)}$ & $i\left(\bar{q}_{p} \gamma^{\mu} \overleftrightarrow{D}^{\nu} q_{r}\right) G_{\mu \rho}^{A} G_{\nu}^{A \rho}$ \\
$Q_{q^{2} G^{2} D}^{(2)}$ & $f^{A B C}\left(\bar{q}_{p} \gamma^{\mu} T^{A} \overleftrightarrow{D}^{\nu} q_{r}\right) G_{\mu \rho}^{B} G_{\nu}^{C \rho}$ \\
$Q_{q^{2} G^{2} D}^{(3)}$ & $i d^{A B C}\left(\bar{q}_{p} \gamma^{\mu} T^{A} \overleftrightarrow{D}^{\nu} q_{r}\right) G_{\mu \rho}^{B} G_{\nu}^{C \rho}$ \\
$Q_{q^{2} W^{2} D}^{(1)}$ & $i\left(\bar{q}_{p} \gamma^{\mu} \overleftrightarrow{D}^{\nu} q_{r}\right) W_{\mu \rho}^{I} W_{\nu}^{I \rho}$ \\
$Q_{q^{2} W^{2} D}^{(2)}$ & $\epsilon^{I J K}\left(\bar{q}_{p} \gamma^{\mu} \tau^{I} \overleftrightarrow{D}^{\nu} q_{r}\right) W_{\mu \rho}^{J} W_{\nu}^{K \rho}$ \\
$Q_{q^{2} B^{2} D}$ & $i\left(\bar{q}_{p} \gamma^{\mu} \overleftrightarrow{D}^{\nu} q_{r}\right) B_{\mu \rho} B_{\nu}{ }^{\rho}$ \\
$Q_{u^{2} G^{2} D}^{(1)}$ & $i\left(\bar{u}_{p} \gamma^{\mu} \overleftrightarrow{D}^{\nu} u_{r}\right) G_{\mu \rho}^{A} G_{\nu}^{A \rho}$ \\
$Q_{u^{2} G^{2} D}^{(2)}$ & $f^{A B C}\left(\bar{u}_{p} \gamma^{\mu} T^{A} \overleftrightarrow{D}^{\nu} u_{r}\right) G_{\mu \rho}^{B} G_{\nu}^{C \rho}$ \\
$Q_{u^{2} G^{2} D}^{(3)}$ & $i d^{A B C}\left(\bar{u}_{p} \gamma^{\mu} T^{A} \overleftrightarrow{D}^{\nu} u_{r}\right) G_{\mu \rho}^{B} G_{\nu}^{C \rho}$ \\
$Q_{u^{2} W^{2} D}$ & $i\left(\bar{u}_{p} \gamma^{\mu} \overleftrightarrow{D}^{\nu} u_{r}\right) W_{\mu \rho}^{I} W_{\nu}^{I \rho}$ \\
$Q_{u^{2} B^{2} D}$ & $i\left(\bar{u}_{p} \gamma^{\mu} \overleftrightarrow{D}^{\nu} u_{r}\right) B_{\mu \rho} B_{\nu}{ }^{\rho}$ \\
$Q_{d^{2} G^{2} D}^{(1)}$ & $i\left(\bar{d}_{p} \gamma^{\mu} \overleftrightarrow{D}^{\nu} d_{r}\right) G_{\mu \rho}^{A} G_{\nu}^{A \rho}$ \\
$Q_{d^{2} G^{2} D}^{(2)}$ & $f^{A B C}\left(\bar{d}_{p} \gamma^{\mu} T^{A} \overleftrightarrow{D}^{\nu} d_{r}\right) G_{\mu \rho}^{B} G_{\nu}^{C \rho}$ \\
$Q_{d^{2} G^{2} D}^{(3)}$ & $i d^{A B C}\left(\bar{d}_{p} \gamma^{\mu} T^{A} \overleftrightarrow{D}^{\nu} d_{r}\right) G_{\mu \rho}^{B} G_{\nu}^{C \rho}$ \\
$Q_{d^{2} W^{2} D}$ & $i\left(\bar{d}_{p} \gamma^{\mu} \overleftrightarrow{D}^{\nu} d_{r}\right) W_{\mu \rho}^{I} W_{\nu}^{I \rho}$ \\
$Q_{d^{2} B^{2} D}$ & $i\left(\bar{d}_{p} \gamma^{\mu} \overleftrightarrow{D}^{\nu} d_{r}\right) B_{\mu \rho} B_{\nu}{ }^{\rho}$ \\
\hline
\end{tabular}

\begin{tabular}{|c|c|}
\hline \multicolumn{2}{|r|}{$14: \psi^{2} X^{2} D$} \\
\hline$Q_{q^{2} G^{2} D}^{(4)}$ & $i f^{A B C}\left(\bar{q}_{p} \gamma^{\mu} T^{A} \overleftrightarrow{D}^{\nu} q_{r}\right)\left(G_{\mu \rho}^{B} \widetilde{G}_{\nu}^{C \rho}-\widetilde{G}_{\mu \rho}^{B} G_{\nu}^{C \rho}\right)$ \\
\hline$Q_{q^{2} G^{2} D}^{(5)}$ & $f^{A B C}\left(\bar{q}_{p} \gamma^{\mu} T^{A} \overleftrightarrow{D}^{\nu} q_{r}\right)\left(G_{\mu \rho}^{B} \widetilde{G}_{\nu}^{C \rho}+\widetilde{G}_{\mu \rho}^{B} G_{\nu}^{C \rho}\right)$ \\
\hline$Q_{q^{2} G W D}^{(1)}$ & $\left(\bar{q}_{p} \gamma^{\mu} T^{A} \tau^{I} \overleftrightarrow{D}^{\nu} q_{r}\right)\left(G_{\mu \rho}^{A} W_{\nu}^{I \rho}-G_{\nu \rho}^{A} W_{\mu}^{I \rho}\right)$ \\
\hline$Q_{q^{2} G W D}^{(2)}$ & $i\left(\bar{q}_{p} \gamma^{\mu} T^{A} \tau^{I} \overleftrightarrow{D}^{\nu} q_{r}\right)\left(G_{\mu \rho}^{A} W_{\nu}^{I \rho}+G_{\nu \rho}^{A} W_{\mu}^{I \rho}\right)$ \\
\hline$Q_{q^{2} G W D}^{(3)}$ & $\left(\bar{q}_{p} \gamma^{\mu} T^{A} \tau^{I} \overleftrightarrow{D}^{\nu} q_{r}\right)\left(G_{\mu \rho}^{A} \widetilde{W}_{\nu}^{I \rho}-G_{\nu \rho}^{A} \widetilde{W}_{\mu}^{I \rho}\right)$ \\
\hline$Q_{q^{2} G W D}^{(4)}$ & $i\left(\bar{q}_{p} \gamma^{\mu} T^{A} \tau^{I} \overleftrightarrow{D}^{\nu} q_{r}\right)\left(G_{\mu \rho}^{A} \widetilde{W}_{\nu}^{I \rho}+G_{\nu \rho}^{A} \widetilde{W}_{\mu}^{I \rho}\right)$ \\
\hline$Q_{q^{2} G B D}^{(1)}$ & $\left(\bar{q}_{p} \gamma^{\mu} \overleftrightarrow{D}^{\nu} q_{r}\right)\left(B_{\mu \rho} G_{\nu}^{A \rho}-B_{\nu \rho} G_{\mu}^{A \rho}\right)$ \\
\hline$Q_{q^{2} G B D}^{(2)}$ & $i\left(\bar{q}_{p} \gamma^{\mu} \overleftrightarrow{D^{\nu}} q_{r}\right)\left(B_{\mu \rho} G_{\nu}^{A \rho}+B_{\nu \rho} G_{\mu}^{A \rho}\right)$ \\
\hline$Q_{q^{2} G B D}^{(3)}$ & $\left(\bar{q}_{p} \gamma^{\mu} \overleftrightarrow{D}^{\nu} q_{r}\right)\left(B_{\mu \rho} \widetilde{G}_{\nu}^{A \rho}-B_{\nu \rho} \widetilde{G}_{\mu}^{A \rho}\right)$ \\
\hline$Q_{q^{2} G B D}^{(4)}$ & $i\left(\bar{q}_{p} \gamma^{\mu} \overleftrightarrow{D}^{\nu} q_{r}\right)\left(B_{\mu \rho} \widetilde{G}_{\nu}^{A \rho}+B_{\nu \rho} \widetilde{G}_{\mu}^{A \rho}\right)$ \\
\hline$Q_{q^{2} W^{2} D}^{(3)}$ & $i \epsilon^{I J K}\left(\bar{q}_{p} \gamma^{\mu} \tau^{I} \overleftrightarrow{D}^{\nu} q_{r}\right)\left(W_{\mu \rho}^{J} \widetilde{W}_{\nu}^{K \rho}-\widetilde{W}_{\mu \rho}^{J} W_{\nu}^{K \rho}\right)$ \\
\hline$Q_{q^{2} W^{2} D}^{(4)}$ & $\epsilon^{I J K}\left(\bar{q}_{p} \gamma^{\mu} \tau^{I} \overleftrightarrow{D}^{\nu} q_{r}\right)\left(W_{\mu \rho}^{J} \widetilde{W}_{\nu}^{K \rho}+\widetilde{W}_{\mu \rho}^{J} W_{\nu}^{K \rho}\right)$ \\
\hline$Q_{q^{2} W B D}^{(1)}$ & $\left(\bar{q}_{p} \gamma^{\mu} \tau^{I} \overleftrightarrow{D}^{\nu} q_{r}\right)\left(B_{\mu \rho} W_{\nu}^{I \rho}-B_{\nu \rho} W_{\mu}^{I \rho}\right)$ \\
\hline$Q_{q^{2} W B D}^{(2)}$ & $i\left(\bar{q}_{p} \gamma^{\mu} \tau^{I} \overleftrightarrow{D}^{\nu} q_{r}\right)\left(B_{\mu \rho} W_{\nu}^{I \rho}+B_{\nu \rho} W_{\mu}^{I \rho}\right)$ \\
\hline$Q_{q^{2} W B D}^{(3)}$ & $\left(\bar{q}_{p} \gamma^{\mu} \tau^{I} \overleftrightarrow{D}^{\nu} q_{r}\right)\left(B_{\mu \rho} \widetilde{W}_{\nu}^{I \rho}-B_{\nu \rho} \widetilde{W}_{\mu}^{I \rho}\right)$ \\
\hline$Q_{q^{2} W B D}^{(4)}$ & $i\left(\bar{q}_{p} \gamma^{\mu} \tau^{I} \overleftrightarrow{D}^{\nu} q_{r}\right)\left(B_{\mu \rho} \widetilde{W}_{\nu}^{I \rho}+B_{\nu \rho} \widetilde{W}_{\mu}^{I \rho}\right)$ \\
\hline$Q_{u^{2} G^{2} D}^{(4)}$ & $i f^{A B C}\left(\bar{u}_{p} \gamma^{\mu} T^{A} \overleftrightarrow{D}^{\nu} u_{r}\right)\left(G_{\mu \rho}^{B} \widetilde{G}_{\nu}^{C \rho}-\widetilde{G}_{\mu \rho}^{B} G_{\nu}^{C \rho}\right)$ \\
\hline$Q_{u^{2} G^{2} D}^{(5)}$ & $f^{A B C}\left(\bar{u}_{p} \gamma^{\mu} T^{A} \overleftrightarrow{D}^{\nu} u_{r}\right)\left(G_{\mu \rho}^{B} \widetilde{G}_{\nu}^{C \rho}+\widetilde{G}_{\mu \rho}^{B} G_{\nu}^{C \rho}\right)$ \\
\hline$Q_{u^{2} G B D}^{(1)}$ & $\left(\bar{u}_{p} \gamma^{\mu} \overleftrightarrow{D}^{\nu} u_{r}\right)\left(B_{\mu \rho} G_{\nu}^{A \rho}-B_{\nu \rho} G_{\mu}^{A \rho}\right)$ \\
\hline$Q_{u^{2} G B D}^{(2)}$ & $i\left(\bar{u}_{p} \gamma^{\mu} \overleftrightarrow{D^{\nu}} u_{r}\right)\left(B_{\mu \rho} G_{\nu}^{A \rho}+B_{\nu \rho} G_{\mu}^{A \rho}\right)$ \\
\hline$Q_{u^{2} G B D}^{(3)}$ & $\left(\bar{u}_{p} \gamma^{\mu} \overleftrightarrow{D}^{\nu} u_{r}\right)\left(B_{\mu \rho} \widetilde{G}_{\nu}^{A \rho}-B_{\nu \rho} \widetilde{G}_{\mu}^{A \rho}\right)$ \\
\hline$Q_{u^{2} G B D}^{(4)}$ & $i\left(\bar{u}_{p} \gamma^{\mu} \overleftrightarrow{D}^{\nu} u_{r}\right)\left(B_{\mu \rho} \widetilde{G}_{\nu}^{A \rho}+B_{\nu \rho} \widetilde{G}_{\mu}^{A \rho}\right)$ \\
\hline$Q_{d^{2} G^{2} D}^{(4)}$ & $i f^{A B C}\left(\bar{d}_{p} \gamma^{\mu} T^{A} \overleftrightarrow{D}^{\nu} d_{r}\right)\left(G_{\mu \rho}^{B} \widetilde{G}_{\nu}^{C \rho}-\widetilde{G}_{\mu \rho}^{B} G_{\nu}^{C \rho}\right)$ \\
\hline$Q_{d^{2} G^{2} D}^{(5)}$ & $f^{A B C}\left(\bar{d}_{p} \gamma^{\mu} T^{A} \overleftrightarrow{D}^{\nu} d_{r}\right)\left(G_{\mu \rho}^{B} \widetilde{G}_{\nu}^{C \rho}+\widetilde{G}_{\mu \rho}^{B} G_{\nu}^{C \rho}\right)$ \\
\hline$Q_{d^{2} G B D}^{(1)}$ & $\left(\bar{d}_{p} \gamma^{\mu} \overleftrightarrow{D}^{\nu} d_{r}\right)\left(B_{\mu \rho} G_{\nu}^{A \rho}-B_{\nu \rho} G_{\mu}^{A \rho}\right)$ \\
\hline$Q_{d^{2} G B D}^{(2)}$ & $i\left(\bar{d}_{p} \gamma^{\mu} \overleftrightarrow{D}^{\nu} d_{r}\right)\left(B_{\mu \rho} G_{\nu}^{A \rho}+B_{\nu \rho} G_{\mu}^{A \rho}\right)$ \\
\hline$Q_{d^{2} G B D}^{(3)}$ & $\left(\bar{d}_{p} \gamma^{\mu} \overleftrightarrow{D}^{\nu} d_{r}\right)\left(B_{\mu \rho} \widetilde{G}_{\nu}^{A \rho}-B_{\nu \rho} \widetilde{G}_{\mu}^{A \rho}\right)$ \\
\hline$Q_{d^{2} G B D}^{(4)}$ & $i\left(\bar{d}_{p} \gamma^{\mu} \overleftrightarrow{D}^{\nu} d_{r}\right)\left(B_{\mu \rho} \widetilde{G}_{\nu}^{A \rho}+B_{\nu \rho} \widetilde{G}_{\mu}^{A \rho}\right)$ \\
\hline
\end{tabular}

Table 6. The hadronic dimension-eight operators in the SMEFT of class-14. The subscripts $p, r$ are weak-eigenstate indices. 


\begin{tabular}{|c|c|}
\hline \multicolumn{2}{|c|}{$\mathbf{1 4}: \boldsymbol{\psi}^{2} \boldsymbol{X}^{2} \boldsymbol{D}$} \\
\hline$Q_{l^{2} G^{2} D}$ & $i\left(\bar{l}_{p} \gamma^{\mu} \overleftrightarrow{D}^{\nu} l_{r}\right) G_{\mu \rho}^{A} G_{\nu}^{A \rho}$ \\
$Q_{l^{2} W^{2} D}^{(1)}$ & $i\left(\bar{l}_{p} \gamma^{\mu} \overleftrightarrow{D}^{\nu} l_{r}\right) W_{\mu \rho}^{I} W_{\nu}^{I \rho}$ \\
$Q_{l^{2} W^{2} D}^{(2)}$ & $\epsilon^{I J K}\left(\bar{l}_{p} \gamma^{\mu} \tau^{I} \overleftrightarrow{D}^{\nu} l_{r}\right) W_{\mu \rho}^{J} W_{\nu}^{K \rho}$ \\
$Q_{l^{2} B^{2} D}$ & $i\left(\bar{l}_{p} \gamma^{\mu} \overleftrightarrow{D}^{\nu} l_{r}\right) B_{\mu \rho} B_{\nu}{ }^{\rho}$ \\
$Q_{e^{2} G^{2} D}$ & $i\left(\bar{e}_{p} \gamma^{\mu} \overleftrightarrow{D}^{\nu} e_{r}\right) G_{\mu \rho}^{A} G_{\nu}^{A \rho}$ \\
$Q_{e^{2} W^{2} D}$ & $i\left(\bar{e}_{p} \gamma^{\mu} \overleftrightarrow{D}^{\nu} e_{r}\right) W_{\mu \rho}^{I} W_{\nu}^{I \rho}$ \\
$Q_{e^{2} B^{2} D}$ & $i\left(\bar{e}_{p} \gamma^{\mu} \overleftrightarrow{D}^{\nu} e_{r}\right) B_{\mu \rho} B_{\nu}{ }^{\rho}$ \\
\hline
\end{tabular}

\begin{tabular}{|c|c|}
\hline \multicolumn{2}{|c|}{$\mathbf{1 5}:(\overline{\boldsymbol{R}} \boldsymbol{R}) \boldsymbol{X} \boldsymbol{H}^{2} \boldsymbol{D}$} \\
\hline$Q_{e^{2} W H^{2} D}^{(1)}$ & $\left(\bar{e}_{p} \gamma^{\nu} e_{r}\right) D^{\mu}\left(H^{\dagger} \tau^{I} H\right) W_{\mu \nu}^{I}$ \\
$Q_{e^{2} W H^{2} D}^{(2)}$ & $\left(\bar{e}_{p} \gamma^{\nu} e_{r}\right) D^{\mu}\left(H^{\dagger} \tau^{I} H\right) \widetilde{W}_{\mu \nu}^{I}$ \\
$Q_{e^{2} W H^{2} D}^{(3)}$ & $\left(\bar{e}_{p} \gamma^{\nu} e_{r}\right)\left(H^{\dagger} \overleftrightarrow{D}^{I \mu} H\right) W_{\mu \nu}^{I}$ \\
$Q_{e^{2} W H^{2} D}^{(4)}$ & $\left(\bar{e}_{p} \gamma^{\nu} e_{r}\right)\left(H^{\dagger} \overleftrightarrow{D}^{I \mu} H\right) \widetilde{W}_{\mu \nu}^{I}$ \\
$Q_{e^{2} B H^{2} D}^{(1)}$ & $\left(\bar{e}_{p} \gamma^{\nu} e_{r}\right) D^{\mu}\left(H^{\dagger} H\right) B_{\mu \nu}$ \\
$Q_{e^{2} B H^{2} D}^{(2)}$ & $\left(\bar{e}_{p} \gamma^{\nu} e_{r}\right) D^{\mu}\left(H^{\dagger} H\right) \widetilde{B}_{\mu \nu}$ \\
$Q_{e^{2} B H^{2} D}^{(3)}$ & $\left(\bar{e}_{p} \gamma^{\nu} e_{r}\right)\left(H^{\dagger} \overleftrightarrow{D}^{\mu} H\right) B_{\mu \nu}$ \\
$Q_{e^{2} B H^{2} D}^{(4)}$ & $\left(\bar{e}_{p} \gamma^{\nu} e_{r}\right)\left(H^{\dagger} \overleftrightarrow{D}^{\mu} H\right) \widetilde{B}_{\mu \nu}$ \\
$Q_{u^{2} G H^{2} D}^{(1)}$ & $\left(\bar{u}_{p} \gamma^{\nu} T^{A} u_{r}\right) D^{\mu}\left(H^{\dagger} H\right) G_{\mu \nu}^{A}$ \\
$Q_{u^{2} G H^{2} D}^{(2)}$ & $\left(\bar{u}_{p} \gamma^{\nu} T^{A} u_{r}\right) D^{\mu}\left(H^{\dagger} H\right) \widetilde{G}_{\mu \nu}^{A}$ \\
$Q_{u^{2} G H^{2} D}^{(3)}$ & $\left(\bar{u}_{p} \gamma^{\nu} T^{A} u_{r}\right)\left(H^{\dagger} \overleftrightarrow{D}^{\mu} H\right) G_{\mu \nu}^{A}$ \\
$Q_{u^{2} G H^{2} D}^{(4)}$ & $\left(\bar{u}_{p} \gamma^{\nu} T^{A} u_{r}\right)\left(H^{\dagger} \overleftrightarrow{D}^{\mu} H\right) \widetilde{G}_{\mu \nu}^{A}$ \\
$Q_{u^{2} W H^{2} D}^{(1)}$ & $\left(\bar{u}_{p} \gamma^{\nu} u_{r}\right) D^{\mu}\left(H^{\dagger} \tau^{I} H\right) W_{\mu \nu}^{I}$ \\
$Q_{u^{2} W H^{2} D}^{(2)}$ & $\left(\bar{u}_{p} \gamma^{\nu} u_{r}\right) D^{\mu}\left(H^{\dagger} \tau^{I} H\right) \widetilde{W}_{\mu \nu}^{I}$ \\
$Q_{u^{2} W H^{2} D}^{(3)}$ & $\left(\bar{u}_{p} \gamma^{\nu} u_{r}\right)\left(H^{\dagger} \overleftrightarrow{D}^{I \mu} H\right) W_{\mu \nu}^{I}$ \\
$Q_{u^{2} W H^{2} D}^{(4)}$ & $\left(\bar{u}_{p} \gamma^{\nu} u_{r}\right)\left(H^{\dagger} \overleftrightarrow{D}^{I \mu} H\right) \widetilde{W}_{\mu \nu}^{I}$ \\
$Q_{u^{2} B H^{2} D}^{(1)}$ & $\left(\bar{u}_{p} \gamma^{\nu} u_{r}\right) D^{\mu}\left(H^{\dagger} H\right) B_{\mu \nu}$ \\
$Q_{u^{2} B H^{2} D}^{(2)}$ & $\left(\bar{u}_{p} \gamma^{\nu} u_{r}\right) D^{\mu}\left(H^{\dagger} H\right) \widetilde{B}_{\mu \nu}$ \\
$Q_{u^{2} B H^{2} D}^{(3)}$ & $\left(\bar{u}_{p} \gamma^{\nu} u_{r}\right)\left(H^{\dagger} \overleftrightarrow{D}^{\mu} H\right) B_{\mu \nu}$ \\
$Q_{u^{2} B H^{2} D}^{(4)}$ & $\left(\bar{u}_{p} \gamma^{\nu} u_{r}\right)\left(H^{\dagger} \overleftrightarrow{D}^{\mu} H\right) \widetilde{B}_{\mu \nu}$ \\
\hline & \\
\hline
\end{tabular}

\begin{tabular}{|c|c|}
\hline \multicolumn{1}{|c|}{$\mathbf{1 4}: \boldsymbol{\psi}^{2} \boldsymbol{X}^{2} \boldsymbol{D}$} \\
\hline$Q_{l^{2} W^{2} D}^{(3)}$ & $i \epsilon^{I J K}\left(\bar{l}_{p} \gamma^{\mu} \tau^{I} \overleftrightarrow{D}^{\nu} l_{r}\right)\left(W_{\mu \rho}^{J} \widetilde{W}_{\nu}^{K \rho}-\widetilde{W}_{\mu \rho}^{J} W_{\nu}^{K \rho}\right)$ \\
$Q_{l^{2} W^{2} D}^{(4)}$ & $\epsilon^{I J K}\left(\bar{l}_{p} \gamma^{\mu} \tau^{I} \overleftrightarrow{D}^{\nu} l_{r}\right)\left(W_{\mu \rho}^{J} \widetilde{W}_{\nu}^{K \rho}+\widetilde{W}_{\mu \rho}^{J} W_{\nu}^{K \rho}\right)$ \\
$Q_{l^{2} W B D}^{(1)}$ & $\left(\bar{l}_{p} \gamma^{\mu} \tau^{I} \overleftrightarrow{D}^{\nu} l_{r}\right)\left(B_{\mu \rho} W_{\nu}^{I \rho}-B_{\nu \rho} W_{\mu}^{I \rho}\right)$ \\
$Q_{l^{2} W B D}^{(2)}$ & $i\left(\bar{l}_{p} \gamma^{\mu} \tau^{I} \overleftrightarrow{D}^{\nu} l_{r}\right)\left(B_{\mu \rho} W_{\nu}^{I \rho}+B_{\nu \rho} W_{\mu}^{I \rho}\right)$ \\
$Q_{l^{2} W B D}^{(3)}$ & $\left(\bar{l}_{p} \gamma^{\mu} \tau^{I} \overleftrightarrow{D}^{\nu} l_{r}\right)\left(B_{\mu \rho} \widetilde{W}_{\nu}^{I \rho}-B_{\nu \rho} \widetilde{W}_{\mu}^{I \rho}\right)$ \\
$Q_{l^{2} W B D}^{(4)}$ & $i\left(\bar{l}_{p} \gamma^{\mu} \tau^{I} \overleftrightarrow{D}^{\nu} l_{r}\right)\left(B_{\mu \rho} \widetilde{W}_{\nu}^{I \rho}+B_{\nu \rho} \widetilde{W}_{\mu}^{I \rho}\right)$ \\
\hline
\end{tabular}

\begin{tabular}{|c|c|}
\hline \multicolumn{2}{|c|}{$15:(\bar{R} R) X H^{2} D$} \\
\hline$Q_{d^{2} G H^{2} D}^{(1)}$ & $\left(\bar{d}_{p} \gamma^{\nu} T^{A} d_{r}\right) D^{\mu}\left(H^{\dagger} H\right) G_{\mu \nu}^{A}$ \\
\hline$Q_{d^{2} G H^{2} D}^{(2)}$ & $\left(\bar{d}_{p} \gamma^{\nu} T^{A} d_{r}\right) D^{\mu}\left(H^{\dagger} H\right) \widetilde{G}_{\mu \nu}^{A}$ \\
\hline$Q_{d^{2} G H^{2} D}^{(3)}$ & $\left(\bar{d}_{p} \gamma^{\nu} T^{A} d_{r}\right)\left(H^{\dagger} \overleftrightarrow{D}^{\mu} H\right) G_{\mu \nu}^{A}$ \\
\hline$Q_{d^{2} G H^{2} D}^{(4)}$ & $\left(\bar{d}_{p} \gamma^{\nu} T^{A} d_{r}\right)\left(H^{\dagger} \overleftrightarrow{D}^{\mu} H\right) \widetilde{G}_{\mu \nu}^{A}$ \\
\hline$Q_{d^{2} W H^{2} D}^{(1)}$ & $\left(\bar{d}_{p} \gamma^{\nu} d_{r}\right) D^{\mu}\left(H^{\dagger} \tau^{I} H\right) W_{\mu \nu}^{I}$ \\
\hline$Q_{d^{2} W H^{2} D}^{(2)}$ & $\left(\bar{d}_{p} \gamma^{\nu} d_{r}\right) D^{\mu}\left(H^{\dagger} \tau^{I} H\right) \widetilde{W}_{\mu \nu}^{I}$ \\
\hline$Q_{d^{2} W H^{2} D}^{(3)}$ & $\left(\bar{d}_{p} \gamma^{\nu} d_{r}\right)\left(H^{\dagger} \overleftrightarrow{D}^{I \mu} H\right) W_{\mu \nu}^{I}$ \\
\hline$Q_{d^{2} W H^{2} D}^{(4)}$ & $\left(\bar{d}_{p} \gamma^{\nu} d_{r}\right)\left(H^{\dagger} \overleftrightarrow{D}^{I \mu} H\right) \widetilde{W}_{\mu \nu}^{I}$ \\
\hline$Q_{d^{2} B H^{2} D}^{(1)}$ & $\left(\bar{d}_{p} \gamma^{\nu} d_{r}\right) D^{\mu}\left(H^{\dagger} H\right) B_{\mu \nu}$ \\
\hline$Q_{d^{2} B H^{2} D}^{(2)}$ & $\left(\bar{d}_{p} \gamma^{\nu} d_{r}\right) D^{\mu}\left(H^{\dagger} H\right) \widetilde{B}_{\mu \nu}$ \\
\hline$Q_{d^{2} B H^{2} D}^{(3)}$ & $\left(\bar{d}_{p} \gamma^{\nu} d_{r}\right)\left(H^{\dagger} \overleftrightarrow{D}^{\mu} H\right) B_{\mu \nu}$ \\
\hline$Q_{d^{2} B H^{2} D}^{(4)}$ & $\left(\bar{d}_{p} \gamma^{\nu} d_{r}\right)\left(H^{\dagger} \overleftrightarrow{D}^{\mu} H\right) \widetilde{B}_{\mu \nu}$ \\
\hline$Q_{u d G H^{2}}^{(1)}+$ h.c. & $\left(\bar{u}_{p} \gamma^{\nu} T^{A} d_{r}\right)\left(\widetilde{H}^{\dagger} \overleftrightarrow{D}^{\mu} H\right) G_{\mu \nu}^{A}$ \\
\hline$Q_{u d G H^{2}}^{(2)}+$ h.c. & $\left(\bar{u}_{p} \gamma^{\nu} T^{A} d_{r}\right)\left(\widetilde{H}^{\dagger} \overleftrightarrow{D}^{\mu} H\right) \widetilde{G}_{\mu \nu}^{A}$ \\
\hline$Q_{u d W H^{2}}^{(1)}+$ h.c. & $\left(\bar{u}_{p} \gamma^{\nu} d_{r}\right)\left(\widetilde{H}^{\dagger} \overleftrightarrow{D}^{I \mu} H\right) W_{\mu \nu}^{I}$ \\
\hline$Q_{u d W H^{2}}^{(2)}+$ h.c. & $\left(\bar{u}_{p} \gamma^{\nu} d_{r}\right)\left(\widetilde{H}^{\dagger} \overleftrightarrow{D}^{I \mu} H\right) \widetilde{W}_{\mu \nu}^{I}$ \\
\hline$Q_{u d B H^{2}}^{(1)}+$ h.c. & $\left(\bar{u}_{p} \gamma^{\nu} d_{r}\right)\left(\widetilde{H}^{\dagger} \overleftrightarrow{D}^{\mu} H\right) B_{\mu \nu}$ \\
\hline$Q_{u d B H^{2}}^{(2)}+$ h.c. & $\left(\bar{u}_{p} \gamma^{\nu} d_{r}\right)\left(\widetilde{H}^{\dagger} \overleftrightarrow{D}^{\mu} H\right) \widetilde{B}_{\mu \nu}$ \\
\hline
\end{tabular}

Table 7. The leptonic dimension-eight operators in the SMEFT of class-14, and the dimension-eight operators of class-15 with field content $(\bar{R} R) X^{2} H$. The operators $Q_{u d X H^{2}}$ have distinct Hermitian conjugates. The subscripts $p, r$ are weak-eigenstate indices. 


\begin{tabular}{|c|c|}
\hline \multicolumn{2}{|r|}{$15:(\bar{L} L) X H^{2} D$} \\
\hline$Q_{l^{2} W H^{2} D}^{(1)}$ & $\left(\bar{l}_{p} \gamma^{\nu} l_{r}\right) D^{\mu}\left(H^{\dagger} \tau^{I} H\right) W_{\mu \nu}^{I}$ \\
\hline$Q_{l^{2} W H^{2} D}^{(2)}$ & $\left(\bar{l}_{p} \gamma^{\nu} l_{r}\right) D^{\mu}\left(H^{\dagger} \tau^{I} H\right) \widetilde{W}_{\mu \nu}^{I}$ \\
\hline$Q_{l^{2} W H^{2} D}^{(3)}$ & $\left(\bar{l}_{p} \gamma^{\nu} l_{r}\right)\left(H^{\dagger} \overleftrightarrow{D}^{I \mu} H\right) W_{\mu \nu}^{I}$ \\
\hline$Q_{l^{2} W H^{2} D}^{(4)}$ & $\left(\bar{l}_{p} \gamma^{\nu} l_{r}\right)\left(H^{\dagger} \overleftrightarrow{D}^{I \mu} H\right) \widetilde{W}_{\mu \nu}^{I}$ \\
\hline$Q_{l^{2} W H^{2} D}^{(5)}$ & $\left(\bar{l}_{p} \gamma^{\nu} \tau^{I} l_{r}\right) D^{\mu}\left(H^{\dagger} H\right) W_{\mu \nu}^{I}$ \\
\hline$Q_{l^{2} W H^{2} D}^{(6)}$ & $\left(\bar{l}_{p} \gamma^{\nu} \tau^{I} l_{r}\right) D^{\mu}\left(H^{\dagger} H\right) \widetilde{W}_{\mu \nu}^{I}$ \\
\hline$Q_{l^{2} W H^{2} D}^{(7)}$ & $\left(\bar{l}_{p} \gamma^{\nu} \tau^{I} l_{r}\right)\left(H^{\dagger} \overleftrightarrow{D}^{\mu} H\right) W_{\mu \nu}^{I}$ \\
\hline$Q_{l^{2} W H^{2} D}^{(8)}$ & $\left(\bar{l}_{p} \gamma^{\nu} \tau^{I} l_{r}\right)\left(H^{\dagger} \overleftrightarrow{D}^{\mu} H\right) \widetilde{W}_{\mu \nu}^{I}$ \\
\hline$Q_{l^{2} W H^{2} D}^{(9)}$ & $\epsilon^{I J K}\left(\bar{l}_{p} \gamma^{\nu} \tau^{I} l_{r}\right) D^{\mu}\left(H^{\dagger} \tau^{J} H\right) W_{\mu \nu}^{K}$ \\
\hline$Q_{l^{2} W H^{2} D}^{(10)}$ & $\epsilon^{I J K}\left(\bar{l}_{p} \gamma^{\nu} \tau^{I} l_{r}\right) D^{\mu}\left(H^{\dagger} \tau^{J} H\right) \widetilde{W}_{\mu \nu}^{K}$ \\
\hline$Q_{l^{2} W H^{2} D}^{(11)}$ & $\epsilon^{I J K}\left(\bar{l}_{p} \gamma^{\nu} \tau^{I} l_{r}\right)\left(H^{\dagger} \overleftrightarrow{D}{ }^{J \mu} H\right) W_{\mu \nu}^{K}$ \\
\hline$Q_{l^{2} W H^{2} D}^{(12)}$ & $\epsilon^{I J K}\left(\bar{l}_{p} \gamma^{\nu} \tau^{I} l_{r}\right)\left(H^{\dagger} \overleftrightarrow{D^{J}}{ }^{J \mu} H\right) \widetilde{W}_{\mu \nu}^{K}$ \\
\hline$Q_{l^{2} B H^{2} D}^{(1)}$ & $\left(\bar{l}_{p} \gamma^{\nu} \tau^{I} l_{r}\right) D^{\mu}\left(H^{\dagger} \tau^{I} H\right) B_{\mu \nu}$ \\
\hline$Q_{l^{2} B H^{2} D}^{(2)}$ & $\left(\bar{l}_{p} \gamma^{\nu} \tau^{I} l_{r}\right) D^{\mu}\left(H^{\dagger} \tau^{I} H\right) \widetilde{B}_{\mu \nu}$ \\
\hline$Q_{l^{2} B H^{2} D}^{(3)}$ & $\left(\bar{l}_{p} \gamma^{\nu} \tau^{I} l_{r}\right)\left(H^{\dagger} \overleftrightarrow{D}^{I \mu} H\right) B_{\mu \nu}$ \\
\hline$Q_{l^{2} B H^{2} D}^{(4)}$ & $\left(\bar{l}_{p} \gamma^{\nu} \tau^{I} l_{r}\right)\left(H^{\dagger} \overleftrightarrow{D}^{I \mu} H\right) \widetilde{B}_{\mu \nu}$ \\
\hline$Q_{l^{2} B H^{2} D}^{(5)}$ & $\left(\bar{l}_{p} \gamma^{\nu} l_{r}\right) D^{\mu}\left(H^{\dagger} H\right) B_{\mu \nu}$ \\
\hline$Q_{l^{2} B H^{2} D}^{(6)}$ & $\left(\bar{l}_{p} \gamma^{\nu} l_{r}\right) D^{\mu}\left(H^{\dagger} H\right) \widetilde{B}_{\mu \nu}$ \\
\hline$Q_{l^{2} B H^{2} D}^{(7)}$ & $\left(\bar{l}_{p} \gamma^{\nu} l_{r}\right)\left(H^{\dagger} \overleftrightarrow{D}^{\mu} H\right) B_{\mu \nu}$ \\
\hline$Q_{l^{2} B H^{2} D}^{(8)}$ & $\left(\bar{l}_{p} \gamma^{\nu} l_{r}\right)\left(H^{\dagger} \overleftrightarrow{D}^{\mu} H\right) \widetilde{B}_{\mu \nu}$ \\
\hline
\end{tabular}

\begin{tabular}{|c|c|}
\hline & $15:(\bar{L} L) X H^{2} D$ \\
\hline$Q_{q^{2} G H^{2} D}^{(1)}$ & $\left(\bar{q}_{p} \gamma^{\nu} T^{A} \tau^{I} q_{r}\right) D^{\mu}\left(H^{\dagger} \tau^{I} H\right) G_{\mu \nu}^{A}$ \\
\hline$Q_{q^{2} G H^{2} D}^{(2)}$ & $\left(\bar{q}_{p} \gamma^{\nu} T^{A} \tau^{I} q_{r}\right) D^{\mu}\left(H^{\dagger} \tau^{I} H\right) \widetilde{G}_{\mu \nu}^{A}$ \\
\hline$Q_{q^{2} G H^{2} D}^{(3)}$ & $\left(\bar{q}_{p} \gamma^{\nu} T^{A} \tau^{I} q_{r}\right)\left(H^{\dagger} \overleftrightarrow{D}^{I \mu} H\right) G_{\mu \nu}^{A}$ \\
\hline$Q_{q^{2} G H^{2} D}^{(4)}$ & $\left(\bar{q}_{p} \gamma^{\nu} T^{A} \tau^{I} q_{r}\right)\left(H^{\dagger} \overleftrightarrow{D}^{I \mu} H\right) \widetilde{G}_{\mu \nu}^{A}$ \\
\hline$Q_{q^{2} G H^{2} D}^{(5)}$ & $\left(\bar{q}_{p} \gamma^{\nu} T^{A} q_{r}\right) D^{\mu}\left(H^{\dagger} H\right) G_{\mu \nu}^{A}$ \\
\hline$Q_{q^{2} G H^{2} D}^{(6)}$ & $\left(\bar{q}_{p} \gamma^{\nu} T^{A} q_{r}\right) D^{\mu}\left(H^{\dagger} H\right) \widetilde{G}_{\mu \nu}^{A}$ \\
\hline$Q_{q^{2} G H^{2} D}^{(7)}$ & $\left(\bar{q}_{p} \gamma^{\nu} T^{A} q_{r}\right)\left(H^{\dagger} \overleftrightarrow{D}^{\mu} H\right) G_{\mu \nu}^{A}$ \\
\hline$Q_{q^{2} G H^{2} D}^{(8)}$ & $\left(\bar{q}_{p} \gamma^{\nu} T^{A} q_{r}\right)\left(H^{\dagger} \overleftrightarrow{D}^{\mu} H\right) \widetilde{G}_{\mu \nu}^{A}$ \\
\hline$Q_{q^{2} W H^{2} D}^{(1)}$ & $\left(\bar{q}_{p} \gamma^{\nu} q_{r}\right) D^{\mu}\left(H^{\dagger} \tau^{I} H\right) W_{\mu \nu}^{I}$ \\
\hline$Q_{q^{2} W H^{2} D}^{(2)}$ & $\left(\bar{q}_{p} \gamma^{\nu} q_{r}\right) D^{\mu}\left(H^{\dagger} \tau^{I} H\right) \widetilde{W}_{\mu \nu}^{I}$ \\
\hline$Q_{q^{2} W H^{2} D}^{(3)}$ & $\left(\bar{q}_{p} \gamma^{\nu} q_{r}\right)\left(H^{\dagger} \overleftrightarrow{D}^{I \mu} H\right) W_{\mu \nu}^{I}$ \\
\hline$Q_{q^{2} W H^{2} D}^{(4)}$ & $\left(\bar{q}_{p} \gamma^{\nu} q_{r}\right)\left(H^{\dagger} \overleftrightarrow{D}^{I \mu} H\right) \widetilde{W}_{\mu \nu}^{I}$ \\
\hline$Q_{q^{2} W H^{2} D}^{(5)}$ & $\left(\bar{q}_{p} \gamma^{\nu} \tau^{I} q_{r}\right) D^{\mu}\left(H^{\dagger} H\right) W_{\mu \nu}^{I}$ \\
\hline$Q_{q^{2} W H^{2} D}^{(6)}$ & $\left(\bar{q}_{p} \gamma^{\nu} \tau^{I} q_{r}\right) D^{\mu}\left(H^{\dagger} H\right) \widetilde{W}_{\mu \nu}^{I}$ \\
\hline$Q_{q^{2} W H^{2} D}^{(7)}$ & $\left(\bar{q}_{p} \gamma^{\nu} \tau^{I} q_{r}\right)\left(H^{\dagger} \overleftrightarrow{D}^{\mu} H\right) W_{\mu \nu}^{I}$ \\
\hline$Q_{q^{2} W H^{2} D}^{(8)}$ & $\left(\bar{q}_{p} \gamma^{\nu} \tau^{I} q_{r}\right)\left(H^{\dagger} \overleftrightarrow{D}^{\mu} H\right) \widetilde{W}_{\mu \nu}^{I}$ \\
\hline$Q_{q^{2} W H^{2} D}^{(9)}$ & $\epsilon^{I J K}\left(\bar{q}_{p} \gamma^{\nu} \tau^{I} q_{r}\right) D^{\mu}\left(H^{\dagger} \tau^{J} H\right) W_{\mu \nu}^{K}$ \\
\hline$Q_{q^{2} W H^{2} D}^{(10)}$ & $\epsilon^{I J K}\left(\bar{q}_{p} \gamma^{\nu} \tau^{I} q_{r}\right) D^{\mu}\left(H^{\dagger} \tau^{J} H\right) \widetilde{W}_{\mu \nu}^{K}$ \\
\hline$Q_{q^{2} W H^{2} D}^{(11)}$ & $\epsilon^{I J K}\left(\bar{q}_{p} \gamma^{\nu} \tau^{I} q_{r}\right)\left(H^{\dagger} \overleftrightarrow{D^{J}}{ }^{J \mu} H\right) W_{\mu \nu}^{K}$ \\
\hline$Q_{q^{2} W H^{2} D}^{(12)}$ & $\epsilon^{I J K}\left(\bar{q}_{p} \gamma^{\nu} \tau^{I} q_{r}\right)\left(H^{\dagger} \overleftrightarrow{D}^{J \mu} H\right) \widetilde{W}_{\mu \nu}^{K}$ \\
\hline$Q_{q^{2} B H^{2} D}^{(1)}$ & $\left(\bar{q}_{p} \gamma^{\nu} \tau^{I} q_{r}\right) D^{\mu}\left(H^{\dagger} \tau^{I} H\right) B_{\mu \nu}$ \\
\hline$Q_{q^{2} B H^{2} D}^{(2)}$ & $\left(\bar{q}_{p} \gamma^{\nu} \tau^{I} q_{r}\right) D^{\mu}\left(H^{\dagger} \tau^{I} H\right) \widetilde{B}_{\mu \nu}$ \\
\hline$Q_{q^{2} B H^{2} D}^{(3)}$ & $\left(\bar{q}_{p} \gamma^{\nu} \tau^{I} q_{r}\right)\left(H^{\dagger} \overleftrightarrow{D}^{I \mu} H\right) B_{\mu \nu}$ \\
\hline$Q_{q^{2} B H^{2} D}^{(4)}$ & $\left(\bar{q}_{p} \gamma^{\nu} \tau^{I} q_{r}\right)\left(H^{\dagger} \overleftrightarrow{D}^{I \mu} H\right) \widetilde{B}_{\mu \nu}$ \\
\hline$Q_{q^{2} B H^{2} D}^{(5)}$ & $\left(\bar{q}_{p} \gamma^{\nu} q_{r}\right) D^{\mu}\left(H^{\dagger} H\right) B_{\mu \nu}$ \\
\hline$Q_{q^{2} B H^{2} D}^{(6)}$ & $\left(\bar{q}_{p} \gamma^{\nu} q_{r}\right) D^{\mu}\left(H^{\dagger} H\right) \widetilde{B}_{\mu \nu}$ \\
\hline$Q_{q^{2} B H^{2} D}^{(7)}$ & $\left(\bar{q}_{p} \gamma^{\nu} q_{r}\right)\left(H^{\dagger} \overleftrightarrow{D}^{\mu} H\right) B_{\mu \nu}$ \\
\hline$Q_{q^{2} B H^{2} D}^{(8)}$ & $\left(\bar{q}_{p} \gamma^{\nu} q_{r}\right)\left(H^{\dagger} \overleftrightarrow{D}^{\mu} H\right) \widetilde{B}_{\mu \nu}$ \\
\hline
\end{tabular}

Table 8. The dimension-eight operators in the SMEFT of class-15 with field content $(\bar{L} L) X^{2} H$. The subscripts $p, r$ are weak-eigenstate indices. 


\begin{tabular}{|c|c|}
\hline \multicolumn{2}{|c|}{$\mathbf{1 6}: \boldsymbol{\psi}^{\mathbf{2}} \boldsymbol{X} \boldsymbol{H} D^{2}+$ h.c. } \\
\hline$Q_{l e W H D^{2}}^{(1)}$ & $\left(\bar{l}_{p} \sigma^{\mu \nu} D^{\rho} e_{r}\right) \tau^{I}\left(D_{\nu} H\right) W_{\rho \mu}^{I}$ \\
$Q_{l e W H D^{2}}^{(2)}$ & $\left(\bar{l}_{p} D^{\rho} e_{r}\right) \tau^{I}\left(D^{\nu} H\right) \widetilde{W}_{\rho \nu}^{I}$ \\
$Q_{l e W H D^{2}}^{(3)}$ & $\left(\bar{l}_{p} \sigma^{\mu \nu} e_{r}\right) \tau^{I}\left(D^{\rho} H\right)\left(D_{\rho} W_{\mu \nu}^{I}\right)$ \\
$Q_{l e B H D^{2}}^{(1)}$ & $\left(\bar{l}_{p} \sigma^{\mu \nu} D^{\rho} e_{r}\right)\left(D_{\nu} H\right) B_{\rho \mu}$ \\
$Q_{l e B H D^{2}}^{(2)}$ & $\left(\bar{l}_{p} D^{\rho} e_{r}\right)\left(D^{\nu} H\right) \widetilde{B}_{\rho \nu}$ \\
$Q_{l e B H D^{2}}^{(3)}$ & $\left(\bar{l}_{p} \sigma^{\mu \nu} e_{r}\right)\left(D^{\rho} H\right)\left(D_{\rho} B_{\mu \nu}\right)$ \\
$Q_{q u G H D^{2}}^{(1)}$ & $\left(\bar{q}_{p} \sigma^{\mu \nu} T^{A} D^{\rho} u_{r}\right)\left(D_{\nu} \widetilde{H}\right) G_{\rho \mu}^{A}$ \\
$Q_{q u G H D^{2}}^{(2)}$ & $\left(\bar{q}_{p} T^{A} D^{\rho} u_{r}\right)\left(D^{\nu} \widetilde{H}\right) \widetilde{G}_{\rho \nu}^{A}$ \\
$Q_{q u G H D^{2}}^{(3)}$ & $\left(\bar{q}_{p} \sigma^{\mu \nu} T^{A} u_{r}\right)\left(D^{\rho} \widetilde{H}\right)\left(D_{\rho} G_{\mu \nu}^{A}\right)$ \\
$Q_{q u W H D^{2}}^{(1)}$ & $\left(\bar{q}_{p} \sigma^{\mu \nu} D^{\rho} u_{r}\right) \tau^{I}\left(D_{\nu} \widetilde{H}\right) W_{\rho \mu}^{I}$ \\
$Q_{q u W H D^{2}}^{(2)}$ & $\left(\bar{q}_{p} D^{\rho} u_{r}\right) \tau^{I}\left(D^{\nu} \widetilde{H}\right) \widetilde{W}_{\rho \nu}^{I}$ \\
$Q_{q u W H D^{2}}^{(3)}$ & $\left(\bar{q}_{p} \sigma^{\mu \nu} u_{r}\right) \tau^{I}\left(D^{\rho} \widetilde{H}\right)\left(D_{\rho} W_{\mu \nu}^{I}\right)$ \\
$Q_{q u B H D^{2}}^{(1)}$ & $\left(\bar{q}_{p} \sigma^{\mu \nu} D^{\rho} u_{r}\right)\left(D_{\nu} \widetilde{H}\right) B_{\rho \mu}$ \\
$Q_{q u B H D^{2}}^{(2)}$ & $\left(\bar{q}_{p} D^{\rho} u_{r}\right)\left(D^{\nu} \widetilde{H}\right) \widetilde{B}_{\rho \nu}$ \\
$Q_{q u B H D^{2}}^{(3)}$ & $\left(\bar{q}_{p} \sigma^{\mu \nu} u_{r}\right)\left(D^{\rho} \widetilde{H}\right)\left(D_{\rho} B_{\mu \nu}\right)$ \\
$Q_{q d G H D^{2}}^{(1)}$ & $\left(\bar{q}_{p} \sigma^{\mu \nu} T^{A} D^{\rho} d_{r}\right)\left(D_{\nu} H\right) G_{\rho \mu}^{A}$ \\
$Q_{q d G H D^{2}}^{(2)}$ & $\left(\bar{q}_{p} T^{A} D^{\rho} d_{r}\right)\left(D^{\nu} H\right) \widetilde{G}_{\rho \nu}^{A}$ \\
$Q_{q d G H D^{2}}^{(3)}$ & $\left(\bar{q}_{p} \sigma^{\mu \nu} T^{A} d_{r}\right)\left(D^{\rho} H\right)\left(D_{\rho} G_{\mu \nu}^{A}\right)$ \\
$Q_{q d W H D^{2}}^{(1)}$ & $\left(\bar{q}_{p} \sigma^{\mu \nu} D^{\rho} d_{r}\right) \tau^{I}\left(D_{\nu} H\right) W_{\rho \mu}^{I}$ \\
$Q_{q d W H D^{2}}^{(2)}$ & $\left(\bar{q}_{p} D^{\rho} d_{r}\right) \tau^{I}\left(D^{\nu} H\right) \widetilde{W}_{\rho \nu}^{I}$ \\
$Q_{q d W H D^{2}}^{(3)}$ & $\left(\bar{q}_{p} \sigma^{\mu \nu} d_{r}\right) \tau^{I}\left(D^{\rho} H\right)\left(D_{\rho} W_{\mu \nu}^{I}\right)$ \\
$Q_{q d B H D^{2}}^{(1)}$ & $\left(\bar{q}_{p} \sigma^{\mu \nu} D^{\rho} d_{r}\right)\left(D_{\nu} H\right) B_{\rho \mu}$ \\
$Q_{q d B H D^{2}}^{(2)}$ & $\left(\bar{q}_{p} D^{\rho} d_{r}\right)\left(D^{\nu} H\right) \widetilde{B}_{\rho \nu}$ \\
$Q_{q d B H D^{2}}^{(3)}$ & $\left(\bar{q}_{p} \sigma^{\mu \nu} d_{r}\right)\left(D^{\rho} H\right)\left(D_{\rho} B_{\mu \nu}\right)$ \\
& \\
&
\end{tabular}

\begin{tabular}{|c|c|}
\hline \multicolumn{2}{|r|}{$17: \psi^{2} H^{3} D^{2}+$ h.c. } \\
\hline$Q_{l e H^{3} D^{2}}^{(1)}$ & $\left(D_{\mu} H^{\dagger} D^{\mu} H\right)\left(\bar{l}_{p} e_{r} H\right)$ \\
\hline$Q_{l e H^{3} D^{2}}^{(2)}$ & $\left(D_{\mu} H^{\dagger} \tau^{I} D^{\mu} H\right)\left(\bar{l}_{p} e_{r} \tau^{I} H\right)$ \\
\hline$Q_{l e H^{3} D^{2}}^{(3)}$ & $\left(D_{\mu} H^{\dagger} D_{\nu} H\right)\left(\bar{l}_{p} \sigma^{\mu \nu} e_{r} H\right)$ \\
\hline$Q_{l e H^{3} D^{2}}^{(4)}$ & $\left(D_{\mu} H^{\dagger} \tau^{I} D_{\nu} H\right)\left(\bar{l}_{p} \sigma^{\mu \nu} e_{r} \tau^{I} H\right)$ \\
\hline$Q_{l e H^{3} D^{2}}^{(5)}$ & $\left(H^{\dagger} D_{\mu} H\right)\left(\bar{l}_{p} e_{r} D^{\mu} H\right)$ \\
\hline$Q_{l e H^{3} D^{2}}^{(6)}$ & $\left(H^{\dagger} D_{\mu} H\right)\left(\bar{l}_{p} \sigma^{\mu \nu} e_{r} D_{\nu} H\right)$ \\
\hline$Q_{q u H^{3} D^{2}}^{(1)}$ & $\left(D_{\mu} H^{\dagger} D^{\mu} H\right)\left(\bar{q}_{p} u_{r} \widetilde{H}\right)$ \\
\hline$Q_{q u H^{3} D^{2}}^{(2)}$ & $\left(D_{\mu} H^{\dagger} \tau^{I} D^{\mu} H\right)\left(\bar{q}_{p} u_{r} \tau^{I} \widetilde{H}\right)$ \\
\hline$Q_{q u H^{3} D^{2}}^{(3)}$ & $\left(D_{\mu} H^{\dagger} D_{\nu} H\right)\left(\bar{q}_{p} \sigma^{\mu \nu} u_{r} \widetilde{H}\right)$ \\
\hline$Q_{q u H^{3} D^{2}}^{(4)}$ & $\left(D_{\mu} H^{\dagger} \tau^{I} D_{\nu} H\right)\left(\bar{q}_{p} \sigma^{\mu \nu} u_{r} \tau^{I} \widetilde{H}\right)$ \\
\hline$Q_{q u H^{3} D^{2}}^{(5)}$ & $\left(D_{\mu} H^{\dagger} H\right)\left(\bar{q}_{p} u_{r} D^{\mu} \widetilde{H}\right)$ \\
\hline$Q_{q u H^{3} D^{2}}^{(6)}$ & $\left(D_{\mu} H^{\dagger} H\right)\left(\bar{q}_{p} \sigma^{\mu \nu} u_{r} D_{\nu} \widetilde{H}\right)$ \\
\hline$Q_{q d H^{3} D^{2}}^{(1)}$ & $\left(D_{\mu} H^{\dagger} D^{\mu} H\right)\left(\bar{q}_{p} d_{r} H\right)$ \\
\hline$Q_{q d H^{3} D^{2}}^{(2)}$ & $\left(D_{\mu} H^{\dagger} \tau^{I} D^{\mu} H\right)\left(\bar{q}_{p} d_{r} \tau^{I} H\right)$ \\
\hline$Q_{q d H^{3} D^{2}}^{(3)}$ & $\left(D_{\mu} H^{\dagger} D_{\nu} H\right)\left(\bar{q}_{p} \sigma^{\mu \nu} d_{r} H\right)$ \\
\hline$Q_{q d H^{3} D^{2}}^{(4)}$ & $\left(D_{\mu} H^{\dagger} \tau^{I} D_{\nu} H\right)\left(\bar{q}_{p} \sigma^{\mu \nu} d_{r} \tau^{I} H\right)$ \\
\hline$Q_{q d H^{3} D^{2}}^{(5)}$ & $\left(H^{\dagger} D_{\mu} H\right)\left(\bar{q}_{p} d_{r} D^{\mu} H\right)$ \\
\hline$Q_{q d H^{3} D^{2}}^{(6)}$ & $\left(H^{\dagger} D_{\mu} H\right)\left(\bar{q}_{p} \sigma^{\mu \nu} d_{r} D_{\nu} H\right)$ \\
\hline
\end{tabular}

Table 9. The dimension-eight operators in the SMEFT of classes-16, and -17, which have two fermions and two derivates. All of the operators have Hermitian conjugates. The subscripts $p, r$ are weak-eigenstate indices. 


\begin{tabular}{|c|c|}
\hline \multicolumn{2}{|c|}{$18:(\bar{L} R)(\bar{R} L) H^{2}+$ h.c. } \\
\hline$Q_{\text {leqdH }}^{(1)}$ & $\left(\bar{l}_{p}^{j} e_{r}\right)\left(\bar{d}_{s} q_{t j}\right)\left(H^{\dagger} H\right)$ \\
\hline$Q_{l e q d H^{2}}^{(2)}$ & $\left(\bar{l}_{p} e_{r}\right) \tau^{I}\left(\bar{d}_{s} q_{t}\right)\left(H^{\dagger} \tau^{I} H\right)$ \\
\hline$Q_{l^{2} u d H^{2}}$ & $\left(\bar{l}_{p} d_{r} H\right)\left(\widetilde{H}^{\dagger} \bar{u}_{s} l_{t}\right)$ \\
\hline$Q_{\text {lequH }}^{(5)}$ & $\left(\bar{l}_{p} e_{r} H\right)\left(\widetilde{H}^{\dagger} \bar{u}_{s} q_{t}\right)$ \\
\hline$Q_{q^{2} u d H^{2}}^{(5)}$ & $\left(\bar{q}_{p} d_{r} H\right)\left(\widetilde{H}^{\dagger} \bar{u}_{s} q_{t}\right)$ \\
\hline$Q_{q^{2} u d H^{2}}^{(6)}$ & $\left(\bar{q}_{p} T^{A} d_{r} H\right)\left(\widetilde{H}^{\dagger} \bar{u}_{s} T^{A} q_{t}\right)$ \\
\hline
\end{tabular}

\begin{tabular}{|c|c|}
\hline \multicolumn{2}{|c|}{$21:(\bar{L} R)(\bar{R} L) D^{2}+$ h.c. } \\
\hline$Q_{\text {leqd }}^{(1)}$ & $D_{\mu}\left(\bar{l}_{p}^{j} e_{r}\right) D^{\mu}\left(\bar{d}_{s}\right.$ \\
\hline$Q_{\text {leqd } D^{2}}^{(2)}$ & $\left(\bar{l}_{p}^{j} \overleftrightarrow{D}_{\mu} e_{r}\right)\left(\bar{d}_{s} \overleftrightarrow{D}^{\mu} q_{t j}\right)$ \\
\hline
\end{tabular}

\begin{tabular}{|c|c|}
\hline \multicolumn{2}{|r|}{$18(\not B): \psi^{4} H^{2}+$ h.c. } \\
\hline$Q_{l q u d H^{2}}^{(1)}$ & $\epsilon_{\alpha \beta \gamma} \epsilon_{j k}\left(d_{p}^{\alpha} C u_{r}^{\beta}\right)\left(q_{s}^{j \gamma} C l_{t}^{k}\right)\left(H^{\dagger} H\right)$ \\
\hline$Q_{l q u d H^{2}}^{(2)}$ & $\epsilon_{\alpha \beta \gamma}\left(\tau^{I} \epsilon\right)_{j k}\left(d_{p}^{\alpha} C u_{r}^{\beta}\right)\left(q_{s}^{j \gamma} C l_{t}^{k}\right)\left(H^{\dagger} \tau^{I} H\right)$ \\
\hline$Q_{e q^{2} u H^{2}}$ & $\epsilon_{\alpha \beta \gamma} \epsilon_{j k}\left(q_{p}^{j \alpha} C q_{r}^{m \beta}\right)\left(u_{s}^{\gamma} C e_{t}\right)\left(H_{m}^{\dagger} H^{k}\right)$ \\
\hline$Q_{l q^{3} H^{2}}^{(1)}$ & $\epsilon_{\alpha \beta \gamma} \epsilon_{m n} \epsilon_{j k}\left(q_{p}^{m \alpha} C q_{r}^{j \beta}\right)\left(q_{s}^{k \gamma} C l_{t}^{n}\right)\left(H^{\dagger} H\right)$ \\
\hline$Q_{l q^{3} H^{2}}^{(2)}$ & $\epsilon_{\alpha \beta \gamma}\left(\tau^{I} \epsilon\right)_{m n} \epsilon_{j k}\left(q_{p}^{m \alpha} C q_{r}^{j \beta}\right)\left(q_{s}^{k \gamma} C l_{t}^{n}\right)\left(H^{\dagger} \tau^{I} H\right)$ \\
\hline$Q_{e u^{2} d H^{2}}$ & $\epsilon_{\alpha \beta \gamma}\left(d_{p}^{\alpha} C u_{r}^{\beta}\right)\left(u_{s}^{\gamma} C e_{t}\right)\left(H^{\dagger} H\right)$ \\
\hline$Q_{l q^{3} H^{2}}^{(3)}$ & $\epsilon_{\alpha \beta \gamma} \epsilon_{m n}\left(\tau^{I} \epsilon\right)_{j k}\left(q_{p}^{m \alpha} C q_{r}^{j \beta}\right)\left(q_{s}^{k \gamma} C l_{t}^{n}\right)\left(H^{\dagger} \tau^{I} H\right)$ \\
\hline$Q_{l q u^{2} H^{2}}$ & $\epsilon_{\alpha \beta \gamma} \epsilon_{j k} \epsilon_{m n}\left(l_{p}^{j} C q_{r}^{m \alpha}\right)\left(u_{s}^{\beta} C u_{t}^{\gamma}\right) \widetilde{H}^{k} \widetilde{H}^{n}$ \\
\hline$Q_{l q d^{2} H^{2}}$ & $\epsilon_{\alpha \beta \gamma} \epsilon_{j k} \epsilon_{m n}\left(l_{p}^{j} q_{r}^{m \alpha}\right)\left(d_{s}^{\beta} C d_{t}^{\gamma}\right) H^{k} H^{n}$ \\
\hline$Q_{e q^{2} d H^{2}}$ & $\epsilon_{\alpha \beta \gamma} \epsilon_{j k} \epsilon_{m n}\left(e_{p} d_{r}^{\alpha}\right)\left(q_{s}^{j \beta} C q_{t}^{m \gamma}\right) \widetilde{H}^{k} \widetilde{H}^{n}$ \\
\hline
\end{tabular}

\begin{tabular}{|c|c|}
\hline \multicolumn{2}{|r|}{$21(\not B): \psi^{4} D^{2}+$ h.c. } \\
\hline$Q_{l q u d D^{2}}^{(1)}$ & $\epsilon_{\alpha \beta \gamma} \epsilon_{j k} D_{\mu}\left(d_{p}^{\alpha} C u_{r}^{\beta}\right) D^{\mu}\left(q_{s}^{j \gamma} C l_{t}^{k}\right)$ \\
\hline$Q_{l q u d D^{2}}^{(2)}$ & $\epsilon_{\alpha \beta \gamma} \epsilon_{j k} D_{\mu}\left(d_{p}^{\alpha} C q_{r}^{j \beta}\right) D^{\mu}\left(u_{s}^{\gamma} C l_{t}^{k}\right)$ \\
\hline$Q_{e q^{2} u D^{2}}$ & $\epsilon_{\alpha \beta \gamma} \epsilon_{j k}\left(q_{p}^{j \alpha} C D_{\mu} q_{r}^{k \beta}\right) D^{\mu}\left(u_{s}^{\gamma} C e_{t}\right)$ \\
\hline$Q_{l q^{3} D^{2}}$ & $\epsilon_{\alpha \beta \gamma} \epsilon_{m n} \epsilon_{j k}\left(q_{p}^{m \alpha} C D_{\mu} q_{r}^{j \beta}\right) D^{\mu}\left(q_{s}^{k \gamma} C l_{t}^{n}\right)$ \\
\hline$Q_{e u^{2} d D^{2}}^{(1)}$ & $\epsilon_{\alpha \beta \gamma}\left(u_{p}^{\alpha} C D_{\mu} u_{r}^{\beta}\right) D^{\mu}\left(d_{s}^{\gamma} C e_{t}\right)$ \\
\hline$Q_{e u^{2} d D^{2}}^{(2)}$ & $\epsilon_{\alpha \beta \gamma}\left(u_{p}^{\alpha} C u_{r}^{\beta}\right)\left(D_{\mu} d_{s}^{\gamma} C D^{\mu} e_{t}\right)$ \\
\hline
\end{tabular}

Table 10. The dimension- 8 operators of classes- 18 and -21 whose fermionic content either has the chiral structure $(\bar{L} R)(\bar{R} L)$ or is baryon number violating. The subscripts $p, r, s, t$ are weak-eigenstate indices. Operators below the dashed lines vanish when there is only one generation of fermions.

Note that sometimes we will explicitly write factors of the cutoff scale of the effective theory, $C_{i} \rightarrow c_{i} / \Lambda^{d-4}$, to better highlight the different orders in the EFT expansion in our phenomenological studies.

\subsection{Light-by-light scattering}

The possibility of non-linear processes involving solely photons had been discussed back in the 1930s [28-31]. Later in the 1950s the cross section for elastic light-by-light scattering was computed in QED [32, 33]. Almost 70 years would pass before this process was finally observed in vacuum in 2019 by the ATLAS collaboration at the LHC [34]. Additionally, the CMS collaboration reports evidence for elastic light-by-light scattering in vacuum [35].

Interactions in the SMEFT involving four photons first start at dimension-8

$$
\mathcal{L}_{L b L}=\frac{\alpha^{2}}{90 M_{e}^{4}}\left[\mathscr{C}_{L b L}^{(1)}\left(F_{\mu \nu} F^{\mu \nu}\right)^{2}+\mathscr{C}_{L b L}^{(2)}\left(F_{\mu \nu} \widetilde{F}^{\mu \nu}\right)^{2}+\widetilde{\mathscr{C}}_{L b L}\left(F_{\mu \nu} F^{\mu \nu}\right)\left(F_{\rho \sigma} \widetilde{F}^{\rho \sigma}\right)\right],
$$




\begin{tabular}{|c|c|}
\hline & $\mathbf{1 8}:(\overline{\boldsymbol{L}} \boldsymbol{L})(\overline{\boldsymbol{L}} \boldsymbol{L}) \boldsymbol{H}^{\mathbf{2}}$ \\
\hline$Q_{l^{4} H^{2}}^{(1)}$ & $\left(\bar{l}_{p} \gamma^{\mu} l_{r}\right)\left(\bar{l}_{s} \gamma_{\mu} l_{t}\right)\left(H^{\dagger} H\right)$ \\
$Q_{l^{4} H^{2}}^{(2)}$ & $\left(\bar{l}_{p} \gamma^{\mu} l_{r}\right)\left(\bar{l}_{s} \gamma_{\mu} \tau^{I} l_{t}\right)\left(H^{\dagger} \tau^{I} H\right)$ \\
$Q_{q^{4} H^{2}}^{(1)}$ & $\left(\bar{q}_{p} \gamma^{\mu} q_{r}\right)\left(\bar{q}_{s} \gamma_{\mu} q_{t}\right)\left(H^{\dagger} H\right)$ \\
$Q_{q^{4} H^{2}}^{(2)}$ & $\left(\bar{q}_{p} \gamma^{\mu} q_{r}\right)\left(\bar{q}_{s} \gamma_{\mu} \tau^{I} q_{t}\right)\left(H^{\dagger} \tau^{I} H\right)$ \\
$Q_{q^{4} H^{2}}^{(3)}$ & $\left(\bar{q}_{p} \gamma^{\mu} \tau^{I} q_{r}\right)\left(\bar{q}_{s} \gamma_{\mu} \tau^{I} q_{t}\right)\left(H^{\dagger} H\right)$ \\
$Q_{l^{2} q^{2} H^{2}}^{(1)}$ & $\left(\bar{l}_{p} \gamma^{\mu} l_{r}\right)\left(\bar{q}_{s} \gamma_{\mu} q_{t}\right)\left(H^{\dagger} H\right)$ \\
$Q_{l^{2} q^{2} H^{2}}^{(2)}$ & $\left(\bar{l}_{p} \gamma^{\mu} \tau^{I} l_{r}\right)\left(\bar{q}_{s} \gamma_{\mu} q_{t}\right)\left(H^{\dagger} \tau^{I} H\right)$ \\
$Q_{l^{2} q^{2} H^{2}}^{(3)}$ & $\left(\bar{l}_{p} \gamma^{\mu} \tau^{I} l_{r}\right)\left(\bar{q}_{s} \gamma_{\mu} \tau^{I} q_{t}\right)\left(H^{\dagger} H\right)$ \\
$Q_{l^{2} q^{2} H^{2}}^{(4)}$ & $\left(\bar{l}_{p} \gamma^{\mu} l_{r}\right)\left(\bar{q}_{s} \gamma_{\mu} \tau^{I} q_{t}\right)\left(H^{\dagger} \tau^{I} H\right)$ \\
$Q_{l^{2} q^{2} H^{2}}^{(5)}$ & $\epsilon^{I J K}\left(\bar{l}_{p} \gamma^{\mu} \tau^{I} l_{r}\right)\left(\bar{q}_{s} \gamma_{\mu} \tau^{J} q_{t}\right)\left(H^{\dagger} \tau^{K} H\right)$ \\
\hline
\end{tabular}

\begin{tabular}{|c|c|}
\hline \multicolumn{2}{|c|}{$\mathbf{1 8}:(\overline{\boldsymbol{R}} \boldsymbol{R})(\overline{\boldsymbol{R}} \boldsymbol{R}) \boldsymbol{H}^{\mathbf{2}}$} \\
\hline$Q_{e^{4} H^{2}}$ & $\left(\bar{e}_{p} \gamma^{\mu} e_{r}\right)\left(\bar{e}_{s} \gamma_{\mu} e_{t}\right)\left(H^{\dagger} H\right)$ \\
$Q_{u^{4} H^{2}}$ & $\left(\bar{u}_{p} \gamma^{\mu} u_{r}\right)\left(\bar{u}_{s} \gamma_{\mu} u_{t}\right)\left(H^{\dagger} H\right)$ \\
$Q_{d^{4} H^{2}}$ & $\left(\bar{d}_{p} \gamma^{\mu} d_{r}\right)\left(\bar{d}_{s} \gamma_{\mu} d_{t}\right)\left(H^{\dagger} H\right)$ \\
$Q_{e^{2} u^{2} H^{2}}$ & $\left(\bar{e}_{p} \gamma^{\mu} e_{r}\right)\left(\bar{u}_{s} \gamma_{\mu} u_{t}\right)\left(H^{\dagger} H\right)$ \\
$Q_{e^{2} d^{2} H^{2}}$ & $\left(\bar{e}_{p} \gamma^{\mu} e_{r}\right)\left(\bar{d}_{s} \gamma_{\mu} d_{t}\right)\left(H^{\dagger} H\right)$ \\
$Q_{u^{2} d^{2} H^{2}}^{(1)}$ & $\left(\bar{u}_{p} \gamma^{\mu} u_{r}\right)\left(\bar{d}_{s} \gamma_{\mu} d_{t}\right)\left(H^{\dagger} H\right)$ \\
$Q_{u^{2} d^{2} H^{2}}^{(2)}$ & $\left(\bar{u}_{p} \gamma^{\mu} T^{A} u_{r}\right)\left(\bar{d}_{s} \gamma_{\mu} T^{A} d_{t}\right)\left(H^{\dagger} H\right)$ \\
\hline
\end{tabular}

\begin{tabular}{|c|c|}
\hline & $\mathbf{1 8}:(\overline{\boldsymbol{L}} \boldsymbol{L})(\overline{\boldsymbol{R}} \boldsymbol{R}) \boldsymbol{H}^{2}$ \\
\hline$Q_{l^{2} e^{2} H^{2}}^{(1)}$ & $\left(\bar{l}_{p} \gamma^{\mu} l_{r}\right)\left(\bar{e}_{s} \gamma_{\mu} e_{t}\right)\left(H^{\dagger} H\right)$ \\
$Q_{l^{2} e^{2} H^{2}}^{(2)}$ & $\left(\bar{l}_{p} \gamma^{\mu} \tau^{I} l_{r}\right)\left(\bar{e}_{s} \gamma_{\mu} e_{t}\right)\left(H^{\dagger} \tau^{I} H\right)$ \\
$Q_{l^{2} u^{2} H^{2}}^{(1)}$ & $\left(\bar{l}_{p} \gamma^{\mu} l_{r}\right)\left(\bar{u}_{s} \gamma_{\mu} u_{t}\right)\left(H^{\dagger} H\right)$ \\
$Q_{l^{2} u^{2} H^{2}}^{(2)}$ & $\left(\bar{l}_{p} \gamma^{\mu} \tau^{I} l_{r}\right)\left(\bar{u}_{s} \gamma_{\mu} u_{t}\right)\left(H^{\dagger} \tau^{I} H\right)$ \\
$Q_{l^{2} d^{2} H^{2}}^{(1)}$ & $\left(\bar{l}_{p} \gamma^{\mu} l_{r}\right)\left(\bar{d}_{s} \gamma_{\mu} d_{t}\right)\left(H^{\dagger} H\right)$ \\
$Q_{l^{2} d^{2} H^{2}}^{(2)}$ & $\left(\bar{l}_{p} \gamma^{\mu} \tau^{I} l_{r}\right)\left(\bar{d}_{s} \gamma_{\mu} d_{t}\right)\left(H^{\dagger} \tau^{I} H\right)$ \\
$Q_{q^{2} e^{2} H^{2}}^{(1)}$ & $\left(\bar{q}_{p} \gamma^{\mu} q_{r}\right)\left(\bar{e}_{s} \gamma_{\mu} e_{t}\right)\left(H^{\dagger} H\right)$ \\
$Q_{q^{2} e^{2} H^{2}}^{(2)}$ & $\left(\bar{q}_{p} \gamma^{\mu} \tau^{I} q_{r}\right)\left(\bar{e}_{s} \gamma_{\mu} e_{t}\right)\left(H^{\dagger} \tau^{I} H\right)$ \\
$Q_{q^{2}}^{(1)}{ }^{2} H^{2}$ & $\left(\bar{q}_{p} \gamma^{\mu} q_{r}\right)\left(\bar{u}_{s} \gamma_{\mu} u_{t}\right)\left(H^{\dagger} H\right)$ \\
$Q_{q^{2} u^{2} H^{2}}^{(2)}$ & $\left(\bar{q}_{p} \gamma^{\mu} \tau^{I} q_{r}\right)\left(\bar{u}_{s} \gamma_{\mu} u_{t}\right)\left(H^{\dagger} \tau^{I} H\right)$ \\
$Q_{q^{2} u^{2} H^{2}}^{(3)}$ & $\left(\bar{q}_{p} \gamma^{\mu} T^{A} q_{r}\right)\left(\bar{u}_{s} \gamma_{\mu} T^{A} u_{t}\right)\left(H^{\dagger} H\right)$ \\
$Q_{q^{2} u^{2} H^{2}}^{(4)}$ & $\left(\bar{q}_{p} \gamma^{\mu} T^{A} \tau^{I} q_{r}\right)\left(\bar{u}_{s} \gamma_{\mu} T^{A} u_{t}\right)\left(H^{\dagger} \tau^{I} H\right)$ \\
$Q_{q^{2} d^{2} H^{2}}^{(1)}$ & $\left(\bar{q}_{p} \gamma^{\mu} q_{r}\right)\left(\bar{d}_{s} \gamma_{\mu} d_{t}\right)\left(H^{\dagger} H\right)$ \\
$Q_{q^{2}{ }^{2} H^{2}}^{(2)}$ & $\left(\bar{q}_{p} \gamma^{\mu} \tau^{I} q_{r}\right)\left(\bar{d}_{s} \gamma_{\mu} d_{t}\right)\left(H^{\dagger} \tau^{I} H\right)$ \\
$Q_{q^{2} d^{2} H^{2}}^{(3)}$ & $\left(\bar{q}_{p} \gamma^{\mu} T^{A} q_{r}\right)\left(\bar{d}_{s} \gamma_{\mu} T^{A} d_{t}\right)\left(H^{\dagger} H\right)$ \\
$Q_{q^{2} d^{2} H^{2}}^{(4)}$ & $\left(\bar{q}_{p} \gamma^{\mu} T^{A} \tau^{I} q_{r}\right)\left(\bar{d}_{s} \gamma_{\mu} T^{A} d_{t}\right)\left(H^{\dagger} \tau^{I} H\right)$ \\
\hline
\end{tabular}

\begin{tabular}{|l|c|}
\hline & $\mathbf{1 8}:(\overline{\boldsymbol{L}} \boldsymbol{R})(\overline{\boldsymbol{L}} \boldsymbol{R}) \boldsymbol{H}^{2}+$ h.c. \\
\hline$Q_{q^{2} u d H^{2}}^{(1)}$ & $\left(\bar{q}_{p}^{j} u_{r}\right) \epsilon_{j k}\left(\bar{q}_{s}^{k} d_{t}\right)\left(H^{\dagger} H\right)$ \\
$Q_{q^{2} u d H^{2}}^{(2)}$ & $\left(\bar{q}_{p}^{j} u_{r}\right)\left(\tau^{I} \epsilon\right)_{j k}\left(\bar{q}_{s}^{k} d_{t}\right)\left(H^{\dagger} \tau^{I} H\right)$ \\
$Q_{q^{2} u d H^{2}}^{(3)}$ & $\left(\bar{q}_{p}^{j} T^{A} u_{r}\right) \epsilon_{j k}\left(\bar{q}_{s}^{k} T^{A} d_{t}\right)\left(H^{\dagger} H\right)$ \\
$Q_{q^{2} u d H^{2}}^{(4)}$ & $\left(\bar{q}_{p}^{j} T^{A} u_{r}\right)\left(\tau^{I} \epsilon\right)_{j k}\left(\bar{q}_{s}^{k} T^{A} d_{t}\right)\left(H^{\dagger} \tau^{I} H\right)$ \\
$Q_{l e q u H^{2}}^{(1)}$ & $\left(\bar{l}_{p}^{j} e_{r}\right) \epsilon_{j k}\left(\bar{q}_{s}^{k} u_{t}\right)\left(H^{\dagger} H\right)$ \\
$Q_{\text {lequH}}^{(2)}$ & $\left(\bar{l}_{p}^{j} e_{r}\right)\left(\tau^{I} \epsilon\right)_{j k}\left(\bar{q}_{s}^{k} u_{t}\right)\left(H^{\dagger} \tau^{I} H\right)$ \\
$Q_{\text {lequH }}^{(3)}$ & $\left(\bar{l}_{p}^{j} \sigma_{\mu \nu} e_{r}\right) \epsilon_{j k}\left(\bar{q}_{s}^{k} \sigma^{\mu \nu} u_{t}\right)\left(H^{\dagger} H\right)$ \\
$Q_{l e q u H^{2}}^{(4)}$ & $\left(\bar{l}_{p}^{j} \sigma_{\mu \nu} e_{r}\right)\left(\tau^{I} \epsilon\right)_{j k}\left(\bar{q}_{s}^{k} \sigma^{\mu \nu} u_{t}\right)\left(H^{\dagger} \tau^{I} H\right)$ \\
$Q_{l^{2} e^{2} H^{2}}^{(3)}$ & $\left(\bar{l}_{p} e_{r} H\right)\left(\bar{l}_{s} e_{t} H\right)$ \\
$Q_{l e q d H^{2}}^{(3)}$ & $\left(\bar{l}_{p} e_{r} H\right)\left(\bar{q}_{s} d_{t} H\right)$ \\
$Q_{l e q d H^{2}}^{(4)}$ & $\left(\bar{l}_{p} e_{r} \tau^{I} H\right)\left(\bar{q}_{s} d_{t} \tau^{I} H\right)$ \\
$Q_{q^{2} u^{2} H^{2}}^{(5)}$ & $\left(\bar{q}_{p} u_{r} \widetilde{H}\right)\left(\bar{q}_{s} u_{t} \widetilde{H}\right)$ \\
$Q_{q^{2} u^{2} H^{2}}^{(6)}$ & $\left(\bar{q}_{p} T^{A} u_{r} \widetilde{H}\right)\left(\bar{q}_{s} T^{A} u_{t} \widetilde{H}\right)$ \\
$Q_{q^{2} d^{2} H^{2}}^{(5)}$ & $\left(\bar{q}_{p} d_{r} H\right)\left(\bar{q}_{s} d_{t} H\right)$ \\
$Q_{q^{2} d^{2} H^{2}}^{(6)}$ & $\left(\bar{q}_{p} T^{A} d_{r} H\right)\left(\bar{q}_{s} T^{A} d_{t} H\right)$ \\
& \\
\hline
\end{tabular}

Table 11. Most of the dimension-eight operators in the SMEFT of class-9, which are further divided into subclasses according to their chiral properties. See table 10 for the remaining class-9 operators. Operators with + h.c. have Hermitian conjugates. The subscripts $p, r, s, t$ are weakeigenstate indices. 


\begin{tabular}{|c|c|}
\hline & $19:(\bar{L} L)(\bar{L} L) X$ \\
\hline$Q_{l^{4} W}^{(1)}$ & $\left(\bar{l}_{p} \gamma^{\mu} l_{r}\right)\left(\bar{l}_{s} \gamma^{\nu} \tau^{I} l_{t}\right) W_{\mu \nu}^{I}$ \\
\hline$Q_{l^{4} W}^{(2)}$ & $\left(\bar{l}_{p} \gamma^{\mu} l_{r}\right)\left(\bar{l}_{s} \gamma^{\nu} \tau^{I} l_{t}\right) \widetilde{W}_{\mu \nu}^{I}$ \\
\hline$Q_{q^{4} G}^{(1)}$ & $\left(\bar{q}_{p} \gamma^{\mu} q_{r}\right)\left(\bar{q}_{s} \gamma^{\nu} T^{A} q_{t}\right) G_{\mu \nu}^{A}$ \\
\hline$Q_{q^{4} G}^{(2)}$ & $\left(\bar{q}_{p} \gamma^{\mu} q_{r}\right)\left(\bar{q}_{s} \gamma^{\nu} T^{A} q_{t}\right) \widetilde{G}_{\mu \nu}^{A}$ \\
\hline$Q_{q^{4} G}^{(3)}$ & $\left(\bar{q}_{p} \gamma^{\mu} \tau^{I} q_{r}\right)\left(\bar{q}_{s} \gamma^{\nu} T^{A} \tau^{I} q_{t}\right) G_{\mu \nu}^{A}$ \\
\hline$Q_{q^{4} G}^{(4)}$ & $\left(\bar{q}_{p} \gamma^{\mu} \tau^{I} q_{r}\right)\left(\bar{q}_{s} \gamma^{\nu} T^{A} \tau^{I} q_{t}\right) \widetilde{G}_{\mu \nu}^{A}$ \\
\hline$Q_{q^{4} W}^{(1)}$ & $\left(\bar{q}_{p} \gamma^{\mu} q_{r}\right)\left(\bar{q}_{s} \gamma^{\nu} \tau^{I} q_{t}\right) W_{\mu \nu}^{I}$ \\
\hline$Q_{q^{4} W}^{(2)}$ & $\left(\bar{q}_{p} \gamma^{\mu} q_{r}\right)\left(\bar{q}_{s} \gamma^{\nu} \tau^{I} q_{t}\right) \widetilde{W}_{\mu \nu}^{I}$ \\
\hline$Q_{q^{4} W}^{(3)}$ & $\left(\bar{q}_{p} \gamma^{\mu} T^{A} q_{r}\right)\left(\bar{q}_{s} \gamma^{\nu} T^{A} \tau^{I} q_{t}\right) W_{\mu \nu}^{I}$ \\
\hline$Q_{q^{4} W}^{(4)}$ & $\left(\bar{q}_{p} \gamma^{\mu} T^{A} q_{r}\right)\left(\bar{q}_{s} \gamma^{\nu} T^{A} \tau^{I} q_{t}\right) \widetilde{W}_{\mu \nu}^{I}$ \\
\hline$Q_{l^{2} q^{2} G}^{(1)}$ & $\left(\bar{l}_{p} \gamma^{\mu} l_{r}\right)\left(\bar{q}_{s} \gamma^{\nu} T^{A} q_{t}\right) G_{\mu \nu}^{A}$ \\
\hline$Q_{l^{2} q^{2} G}^{(2)}$ & $\left(\bar{l}_{p} \gamma^{\mu} l_{r}\right)\left(\bar{q}_{s} \gamma^{\nu} T^{A} q_{t}\right) \widetilde{G}_{\mu \nu}^{A}$ \\
\hline$Q_{l^{2} q^{2} G}^{(3)}$ & $\left(\bar{l}_{p} \gamma^{\mu} \tau^{I} l_{r}\right)\left(\bar{q}_{s} \gamma^{\nu} T^{A} \tau^{I} q_{t}\right) G_{\mu \nu}^{A}$ \\
\hline$Q_{l^{2} q^{2} G}^{(4)}$ & $\left(\bar{l}_{p} \gamma^{\mu} \tau^{I} l_{r}\right)\left(\bar{q}_{s} \gamma^{\nu} T^{A} \tau^{I} q_{t}\right) \widetilde{G}_{\mu \nu}^{A}$ \\
\hline$Q_{l^{2} q^{2} W}^{(1)}$ & $\left(\bar{l}_{p} \gamma^{\mu} l_{r}\right)\left(\bar{q}_{s} \gamma^{\nu} \tau^{I} q_{t}\right) W_{\mu \nu}^{I}$ \\
\hline$Q_{l^{2} q^{2} W}^{(2)}$ & $\left(\bar{l}_{p} \gamma^{\mu} l_{r}\right)\left(\bar{q}_{s} \gamma^{\nu} \tau^{I} q_{t}\right) \widetilde{W}_{\mu \nu}^{I}$ \\
\hline$Q_{l^{2} q^{2} W}^{(3)}$ & $\left(\bar{l}_{p} \gamma^{\mu} \tau^{I} l_{r}\right)\left(\bar{q}_{s} \gamma^{\nu} q_{t}\right) W_{\mu \nu}^{I}$ \\
\hline$Q_{l^{2} q^{2} W}^{(4)}$ & $\left(\bar{l}_{p} \gamma^{\mu} \tau^{I} l_{r}\right)\left(\bar{q}_{s} \gamma^{\nu} q_{t}\right) \widetilde{W}_{\mu \nu}^{I}$ \\
\hline$Q_{l^{2} q^{2} W}^{(5)}$ & $\epsilon^{I J K}\left(\bar{l}_{p} \gamma^{\mu} \tau^{I} l_{r}\right)\left(\bar{q}_{s} \gamma^{\nu} \tau^{J} q_{t}\right) W_{\mu \nu}^{K}$ \\
\hline$Q_{l^{2} q^{2} W}^{(6)}$ & $\epsilon^{I J K}\left(\bar{l}_{p} \gamma^{\mu} \tau^{I} l_{r}\right)\left(\bar{q}_{s} \gamma^{\nu} \tau^{J} q_{t}\right) \widetilde{W}_{\mu \nu}^{K}$ \\
\hline$Q_{l^{2} q^{2} B}^{(1)}$ & $\left(\bar{l}_{p} \gamma^{\mu} l_{r}\right)\left(\bar{q}_{s} \gamma^{\nu} q_{t}\right) B_{\mu \nu}$ \\
\hline$Q_{l^{2} q^{2} B}^{(2)}$ & $\left(\bar{l}_{p} \gamma^{\mu} l_{r}\right)\left(\bar{q}_{s} \gamma^{\nu} q_{t}\right) \widetilde{B}_{\mu \nu}$ \\
\hline$Q_{l^{2} q^{2} B}^{(3)}$ & $\left(\bar{l}_{p} \gamma^{\mu} \tau^{I} l_{r}\right)\left(\bar{q}_{s} \gamma^{\nu} \tau^{I} q_{t}\right) B_{\mu \nu}$ \\
\hline$Q_{-\underline{l}^{2} \underline{q}^{2} B}^{(4)}$ & $\left(\bar{l}_{p} \gamma^{\mu} \tau^{I} l_{r}\right)\left(\bar{q}_{s} \gamma^{\nu} \tau^{I} q_{t}\right) \widetilde{B}_{\mu \nu}$ \\
\hline$Q_{l^{4} B}^{(1)}$ & $\left(\bar{l}_{p} \gamma^{\mu} l_{r}\right)\left(\bar{l}_{s} \gamma^{\nu} l_{t}\right) B_{\mu \nu}$ \\
\hline$Q_{l^{4} B}^{(2)}$ & $\left(\bar{l}_{p} \gamma^{\mu} l_{r}\right)\left(\bar{l}_{s} \gamma^{\nu} l_{t}\right) \widetilde{B}_{\mu \nu}$ \\
\hline$Q_{q^{4} B}^{(1)}$ & $\left(\bar{q}_{p} \gamma^{\mu} q_{r}\right)\left(\bar{q}_{s} \gamma^{\nu} q_{t}\right) B_{\mu \nu}$ \\
\hline$Q_{q^{4} B}^{(2)}$ & $\left(\bar{q}_{p} \gamma^{\mu} q_{r}\right)\left(\bar{q}_{s} \gamma^{\nu} q_{t}\right) \widetilde{B}_{\mu \nu}$ \\
\hline$Q_{q^{4} B}^{(3)}$ & $\left(\bar{q}_{p} \gamma^{\mu} \tau^{I} q_{r}\right)\left(\bar{q}_{s} \gamma^{\nu} \tau^{I} q_{t}\right) B_{\mu \nu}$ \\
\hline$Q_{q^{4} B}^{(4)}$ & $\left(\bar{q}_{p} \gamma^{\mu} \tau^{I} q_{r}\right)\left(\bar{q}_{s} \gamma^{\nu} \tau^{I} q_{t}\right) \widetilde{B}_{\mu \nu}$ \\
\hline
\end{tabular}

\begin{tabular}{|c|c|}
\hline \multicolumn{2}{|r|}{$19:(\bar{R} R)(\bar{R} R) X$} \\
\hline$Q_{u^{4} G}^{(1)}$ & $\left(\bar{u}_{p} \gamma^{\mu} u_{r}\right)\left(\bar{u}_{s} \gamma^{\nu} T^{A} u_{t}\right) G_{\mu \nu}^{A}$ \\
\hline$Q_{u^{4} G}^{(2)}$ & $\left(\bar{u}_{p} \gamma^{\mu} u_{r}\right)\left(\bar{u}_{s} \gamma^{\nu} T^{A} u_{t}\right) \widetilde{G}_{\mu \nu}^{A}$ \\
\hline$Q_{d^{4} G}^{(1)}$ & $\left(\bar{d}_{p} \gamma^{\mu} d_{r}\right)\left(\bar{d}_{s} \gamma^{\nu} T^{A} d_{t}\right) G_{\mu \nu}^{A}$ \\
\hline$Q_{d^{4} G}^{(2)}$ & $\left(\bar{d}_{p} \gamma^{\mu} d_{r}\right)\left(\bar{d}_{s} \gamma^{\nu} T^{A} d_{t}\right) \widetilde{G}_{\mu \nu}^{A}$ \\
\hline$Q_{e^{2} u^{2} G}^{(1)}$ & $\left(\bar{e}_{p} \gamma^{\mu} e_{r}\right)\left(\bar{u}_{s} \gamma^{\nu} T^{A} u_{t}\right) G_{\mu \nu}^{A}$ \\
\hline$Q_{e^{2} u^{2} G}^{(2)}$ & $\left(\bar{e}_{p} \gamma^{\mu} e_{r}\right)\left(\bar{u}_{s} \gamma^{\nu} T^{A} u_{t}\right) \widetilde{G}_{\mu \nu}^{A}$ \\
\hline$Q_{e^{2} u^{2} B}^{(1)}$ & $\left(\bar{e}_{p} \gamma^{\mu} e_{r}\right)\left(\bar{u}_{s} \gamma^{\nu} u_{t}\right) B_{\mu \nu}$ \\
\hline$Q_{e^{2} u^{2} B}^{(2)}$ & $\left(\bar{e}_{p} \gamma^{\mu} e_{r}\right)\left(\bar{u}_{s} \gamma^{\nu} u_{t}\right) \widetilde{B}_{\mu \nu}$ \\
\hline$Q_{e^{2} d^{2} G}^{(1)}$ & $\left(\bar{e}_{p} \gamma^{\mu} e_{r}\right)\left(\bar{d}_{s} \gamma^{\nu} T^{A} d_{t}\right) G_{\mu \nu}^{A}$ \\
\hline$Q_{e^{2} d^{2} G}^{(2)}$ & $\left(\bar{e}_{p} \gamma^{\mu} e_{r}\right)\left(\bar{d}_{s} \gamma^{\nu} T^{A} d_{t}\right) \widetilde{G}_{\mu \nu}^{A}$ \\
\hline$Q_{e^{2} d^{2} B}^{(1)}$ & $\left(\bar{e}_{p} \gamma^{\mu} e_{r}\right)\left(\bar{d}_{s} \gamma^{\nu} d_{t}\right) B_{\mu \nu}$ \\
\hline$Q_{e^{2} d^{2} B}^{(2)}$ & $\left(\bar{e}_{p} \gamma^{\mu} e_{r}\right)\left(\bar{d}_{s} \gamma^{\nu} d_{t}\right) \widetilde{B}_{\mu \nu}$ \\
\hline$Q_{u^{2} d^{2} G}^{(1)}$ & $\left(\bar{u}_{p} \gamma^{\mu} u_{r}\right)\left(\bar{d}_{s} \gamma^{\nu} T^{A} d_{t}\right) G_{\mu \nu}^{A}$ \\
\hline$Q_{u^{2} d^{2} G}^{(2)}$ & $\left(\bar{u}_{p} \gamma^{\mu} u_{r}\right)\left(\bar{d}_{s} \gamma^{\nu} T^{A} d_{t}\right) \widetilde{G}_{\mu \nu}^{A}$ \\
\hline$Q_{u^{2} d^{2} G}^{(3)}$ & $\left(\bar{u}_{p} \gamma^{\mu} T^{A} u_{r}\right)\left(\bar{d}_{s} \gamma^{\nu} d_{t}\right) G_{\mu \nu}^{A}$ \\
\hline$Q_{u^{2} d^{2} G}^{(4)}$ & $\left(\bar{u}_{p} \gamma^{\mu} T^{A} u_{r}\right)\left(\bar{d}_{s} \gamma^{\nu} d_{t}\right) \widetilde{G}_{\mu \nu}^{A}$ \\
\hline$Q_{u^{2} d^{2} G}^{(5)}$ & $f^{A B C}\left(\bar{u}_{p} \gamma^{\mu} T^{A} u_{r}\right)\left(\bar{d}_{s} \gamma^{\nu} T^{B} d_{t}\right) G_{\mu \nu}^{C}$ \\
\hline$Q_{u^{2} d^{2} G}^{(6)}$ & $f^{A B C}\left(\bar{u}_{p} \gamma^{\mu} T^{A} u_{r}\right)\left(\bar{d}_{s} \gamma^{\nu} T^{B} d_{t}\right) \widetilde{G}_{\mu \nu}^{C}$ \\
\hline$Q_{u^{2} d^{2} G}^{(7)}$ & $d^{A B C}\left(\bar{u}_{p} \gamma^{\mu} T^{A} u_{r}\right)\left(\bar{d}_{s} \gamma^{\nu} T^{B} d_{t}\right) G_{\mu \nu}^{C}$ \\
\hline$Q_{u^{2} d^{2} G}^{(8)}$ & $d^{A B C}\left(\bar{u}_{p} \gamma^{\mu} T^{A} u_{r}\right)\left(\bar{d}_{s} \gamma^{\nu} T^{B} d_{t}\right) \widetilde{G}_{\mu \nu}^{C}$ \\
\hline$Q_{u^{2} d^{2} B}^{(1)}$ & $\left(\bar{u}_{p} \gamma^{\mu} u_{r}\right)\left(\bar{d}_{s} \gamma^{\nu} d_{t}\right) B_{\mu \nu}$ \\
\hline$Q_{u^{2} d^{2} B}^{(2)}$ & $\left(\bar{u}_{p} \gamma^{\mu} u_{r}\right)\left(\bar{d}_{s} \gamma^{\nu} d_{t}\right) \widetilde{B}_{\mu \nu}$ \\
\hline$Q_{u^{2} d^{2} B}^{(3)}$ & $\left(\bar{u}_{p} \gamma^{\mu} T^{A} u_{r}\right)\left(\bar{d}_{s} \gamma^{\nu} T^{A} d_{t}\right) B_{\mu \nu}$ \\
\hline$Q_{\underline{u}^{2} \underline{d}^{2} B}^{(4)}$ & $\left(\bar{u}_{p} \gamma^{\mu} T^{A} u_{r}\right)\left(\bar{d}_{s} \gamma^{\nu} T^{A} d_{t}\right) \widetilde{B}_{\mu \nu}$ \\
\hline$Q_{e^{4} B}^{(1)}$ & $\left(\bar{e}_{p} \gamma^{\mu} e_{r}\right)\left(\bar{e}_{s} \gamma^{\nu} e_{t}\right) B_{\mu \nu}$ \\
\hline$Q_{e^{4} B}^{(2)}$ & $\left(\bar{e}_{p} \gamma^{\mu} e_{r}\right)\left(\bar{e}_{s} \gamma^{\nu} e_{t}\right) \widetilde{B}_{\mu \nu}$ \\
\hline$Q_{u^{4} B}^{(1)}$ & $\left(\bar{u}_{p} \gamma^{\mu} u_{r}\right)\left(\bar{u}_{s} \gamma^{\nu} u_{t}\right) B_{\mu \nu}$ \\
\hline$Q_{u^{4} B}^{(2)}$ & $\left(\bar{u}_{p} \gamma^{\mu} u_{r}\right)\left(\bar{u}_{s} \gamma^{\nu} u_{t}\right) \widetilde{B}_{\mu \nu}$ \\
\hline$Q_{d^{4} B}^{(1)}$ & $\left(\bar{d}_{p} \gamma^{\mu} d_{r}\right)\left(\bar{d}_{s} \gamma^{\nu} d_{t}\right) B_{\mu \nu}$ \\
\hline$Q_{d^{4} B}^{(2)}$ & $\left(\bar{d}_{p} \gamma^{\mu} d_{r}\right)\left(\bar{d}_{s} \gamma^{\nu} d_{t}\right) \widetilde{B}_{\mu \nu}$ \\
\hline
\end{tabular}

Table 12. The dimension-eight operators in the SMEFT of class-19 with field content $J J X$ with $J=(\bar{L} L)$ or $(\bar{R} R)$. The subscripts $p, r, s, t$ are weak-eigenstate indices. Operators below the dashed lines vanish when there is only one generation of fermions. 


\begin{tabular}{|c|c|}
\hline \multicolumn{2}{|r|}{$19:(\bar{L} L)(\bar{R} R) X$} \\
\hline$Q_{l^{2} e^{2} W}^{(1)}$ & $\left(\bar{l}_{p} \gamma^{\mu} \tau^{I} l_{r}\right)\left(\bar{e}_{s} \gamma^{\nu} e_{t}\right) W_{\mu \nu}^{I}$ \\
\hline$Q_{l^{2} e^{2} W}^{(2)}$ & $\left(\bar{l}_{p} \gamma^{\mu} \tau^{I} l_{r}\right)\left(\bar{e}_{s} \gamma^{\nu} e_{t}\right) \widetilde{W}_{\mu \nu}^{I}$ \\
\hline$Q_{l^{2} e^{2} B}^{(1)}$ & $\left(\bar{l}_{p} \gamma^{\mu} l_{r}\right)\left(\bar{e}_{s} \gamma^{\nu} e_{t}\right) B_{\mu \nu}$ \\
\hline$Q_{l^{2} e^{2} B}^{(2)}$ & $\left(\bar{l}_{p} \gamma^{\mu} l_{r}\right)\left(\bar{e}_{s} \gamma^{\nu} e_{t}\right) \widetilde{B}_{\mu \nu}$ \\
\hline$Q_{l^{2} u^{2} G}^{(1)}$ & $\left(\bar{l}_{p} \gamma^{\mu} l_{r}\right)\left(\bar{u}_{s} \gamma^{\nu} T^{A} u_{t}\right) G_{\mu \nu}^{A}$ \\
\hline$Q_{l^{2} u^{2} G}^{(2)}$ & $\left(\bar{l}_{p} \gamma^{\mu} l_{r}\right)\left(\bar{u}_{s} \gamma^{\nu} T^{A} u_{t}\right) \widetilde{G}_{\mu \nu}^{A}$ \\
\hline$Q_{l^{2} u^{2} W}^{(1)}$ & $\left(\bar{l}_{p} \gamma^{\mu} \tau^{I} l_{r}\right)\left(\bar{u}_{s} \gamma^{\nu} u_{t}\right) W_{\mu \nu}^{I}$ \\
\hline$Q_{l^{2} u^{2} W}^{(2)}$ & $\left(\bar{l}_{p} \gamma^{\mu} \tau^{I} l_{r}\right)\left(\bar{u}_{s} \gamma^{\nu} u_{t}\right) \widetilde{W}_{\mu \nu}^{I}$ \\
\hline$Q_{l^{2} u^{2} B}^{(1)}$ & $\left(\bar{l}_{p} \gamma^{\mu} l_{r}\right)\left(\bar{u}_{s} \gamma^{\nu} u_{t}\right) B_{\mu \nu}$ \\
\hline$Q_{l^{2} u^{2} B}^{(2)}$ & $\left(\bar{l}_{p} \gamma^{\mu} l_{r}\right)\left(\bar{u}_{s} \gamma^{\nu} u_{t}\right) \widetilde{B}_{\mu \nu}$ \\
\hline$Q_{l^{2} d^{2} G}^{(1)}$ & $\left(\bar{l}_{p} \gamma^{\mu} l_{r}\right)\left(\bar{d}_{s} \gamma^{\nu} T^{A} d_{t}\right) G_{\mu \nu}^{A}$ \\
\hline$Q_{l^{2} d^{2} G}^{(2)}$ & $\left(\bar{l}_{p} \gamma^{\mu} l_{r}\right)\left(\bar{d}_{s} \gamma^{\nu} T^{A} d_{t}\right) \widetilde{G}_{\mu \nu}^{A}$ \\
\hline$Q_{l^{2} d^{2} W}^{(1)}$ & $\left(\bar{l}_{p} \gamma^{\mu} \tau^{I} l_{r}\right)\left(\bar{d}_{s} \gamma^{\nu} d_{t}\right) W_{\mu \nu}^{I}$ \\
\hline$Q_{l^{2} d^{2} W}^{(2)}$ & $\left(\bar{l}_{p} \gamma^{\mu} \tau^{I} l_{r}\right)\left(\bar{d}_{s} \gamma^{\nu} d_{t}\right) \widetilde{W}_{\mu \nu}^{I}$ \\
\hline$Q_{l^{2} d^{2} B}^{(1)}$ & $\left(\bar{l}_{p} \gamma^{\mu} l_{r}\right)\left(\bar{d}_{s} \gamma^{\nu} d_{t}\right) B_{\mu \nu}$ \\
\hline$Q_{l^{2} d^{2} B}^{(2)}$ & $\left(\bar{l}_{p} \gamma^{\mu} l_{r}\right)\left(\bar{d}_{s} \gamma^{\nu} d_{t}\right) \widetilde{B}_{\mu \nu}$ \\
\hline$Q_{q^{2} e^{2} G}^{(1)}$ & $\left(\bar{q}_{p} \gamma^{\mu} T^{A} q_{r}\right)\left(\bar{e}_{s} \gamma^{\nu} e_{t}\right) G_{\mu \nu}^{A}$ \\
\hline$Q_{q^{2} e^{2} G}^{(2)}$ & $\left(\bar{q}_{p} \gamma^{\mu} T^{A} q_{r}\right)\left(\bar{e}_{s} \gamma^{\nu} e_{t}\right) \widetilde{G}_{\mu \nu}^{A}$ \\
\hline$Q_{q^{2} e^{2} W}^{(1)}$ & $\left(\bar{q}_{p} \gamma^{\mu} \tau^{I} q_{r}\right)\left(\bar{e}_{s} \gamma^{\nu} e_{t}\right) W_{\mu \nu}^{I}$ \\
\hline$Q_{q^{2} e^{2} W}^{(2)}$ & $\left(\bar{q}_{p} \gamma^{\mu} \tau^{I} q_{r}\right)\left(\bar{e}_{s} \gamma^{\nu} e_{t}\right) \widetilde{W}_{\mu \nu}^{I}$ \\
\hline$Q_{q^{2} e^{2} B}^{(1)}$ & $\left(\bar{q}_{p} \gamma^{\mu} q_{r}\right)\left(\bar{e}_{s} \gamma^{\nu} e_{t}\right) B_{\mu \nu}$ \\
\hline$Q_{q^{2} e^{2} B}^{(2)}$ & $\left(\bar{q}_{p} \gamma^{\mu} q_{r}\right)\left(\bar{e}_{s} \gamma^{\nu} e_{t}\right) \widetilde{B}_{\mu \nu}$ \\
\hline$Q_{q^{2} u^{2} G}^{(1)}$ & $\left(\bar{q}_{p} \gamma^{\mu} q_{r}\right)\left(\bar{u}_{s} \gamma^{\nu} T^{A} u_{t}\right) G_{\mu \nu}^{A}$ \\
\hline$Q_{q^{2} u^{2} G}^{(2)}$ & $\left(\bar{q}_{p} \gamma^{\mu} q_{r}\right)\left(\bar{u}_{s} \gamma^{\nu} T^{A} u_{t}\right) \widetilde{G}_{\mu \nu}^{A}$ \\
\hline$Q_{q^{2} u^{2} G}^{(3)}$ & $\left(\bar{q}_{p} \gamma^{\mu} T^{A} q_{r}\right)\left(\bar{u}_{s} \gamma^{\nu} u_{t}\right) G_{\mu \nu}^{A}$ \\
\hline$Q_{q^{2} u^{2} G}^{(4)}$ & $\left(\bar{q}_{p} \gamma^{\mu} T^{A} q_{r}\right)\left(\bar{u}_{s} \gamma^{\nu} u_{t}\right) \widetilde{G}_{\mu \nu}^{A}$ \\
\hline
\end{tabular}

\begin{tabular}{|c|c|}
\hline & $19:(\bar{L} L)(\bar{R} R) X$ \\
\hline$Q_{q^{2} u^{2} G}^{(5)}$ & $f^{A B C}\left(\bar{q}_{p} \gamma^{\mu} T^{A} q_{r}\right)\left(\bar{u}_{s} \gamma^{\nu} T^{B} u_{t}\right) G_{\mu \nu}^{C}$ \\
\hline$Q_{q^{2} u^{2} G}^{(6)}$ & $f^{A B C}\left(\bar{q}_{p} \gamma^{\mu} T^{A} q_{r}\right)\left(\bar{u}_{s} \gamma^{\nu} T^{B} u_{t}\right) \widetilde{G}_{\mu \nu}^{C}$ \\
\hline$Q_{q^{2} u^{2} G}^{(7)}$ & $d^{A B C}\left(\bar{q}_{p} \gamma^{\mu} T^{A} q_{r}\right)\left(\bar{u}_{s} \gamma^{\nu} T^{B} u_{t}\right) G_{\mu \nu}^{C}$ \\
\hline$Q_{q^{2} u^{2} G}^{(8)}$ & $d^{A B C}\left(\bar{q}_{p} \gamma^{\mu} T^{A} q_{r}\right)\left(\bar{u}_{s} \gamma^{\nu} T^{B} u_{t}\right) \widetilde{G}_{\mu \nu}^{C}$ \\
\hline$Q_{q^{2} u^{2} W}^{(1)}$ & $\left(\bar{q}_{p} \gamma^{\mu} \tau^{I} q_{r}\right)\left(\bar{u}_{s} \gamma^{\nu} u_{t}\right) W_{\mu \nu}^{I}$ \\
\hline$Q_{q^{2} u^{2} W}^{(2)}$ & $\left(\bar{q}_{p} \gamma^{\mu} \tau^{I} q_{r}\right)\left(\bar{u}_{s} \gamma^{\nu} u_{t}\right) \widetilde{W}_{\mu \nu}^{I}$ \\
\hline$Q_{q^{2} u^{2} W}^{(3)}$ & $\left(\bar{q}_{p} \gamma^{\mu} T^{A} \tau^{I} q_{r}\right)\left(\bar{u}_{s} \gamma^{\nu} T^{A} u_{t}\right) W_{\mu \nu}^{I}$ \\
\hline$Q_{q^{2} u^{2} W}^{(4)}$ & $\left(\bar{q}_{p} \gamma^{\mu} T^{A} \tau^{I} q_{r}\right)\left(\bar{u}_{s} \gamma^{\nu} T^{A} u_{t}\right) \widetilde{W}_{\mu \nu}^{I}$ \\
\hline$Q_{q^{2} u^{2} B}^{(1)}$ & $\left(\bar{q}_{p} \gamma^{\mu} q_{r}\right)\left(\bar{u}_{s} \gamma^{\nu} u_{t}\right) B_{\mu \nu}$ \\
\hline$Q_{q^{2} u^{2} B}^{(2)}$ & $\left(\bar{q}_{p} \gamma^{\mu} q_{r}\right)\left(\bar{u}_{s} \gamma^{\nu} u_{t}\right) \widetilde{B}_{\mu \nu}$ \\
\hline$Q_{q^{2} u^{2} B}^{(3)}$ & $\left(\bar{q}_{p} \gamma^{\mu} T^{A} q_{r}\right)\left(\bar{u}_{s} \gamma^{\nu} T^{A} u_{t}\right) B_{\mu \nu}$ \\
\hline$Q_{q^{2} u^{2} B}^{(4)}$ & $\left(\bar{q}_{p} \gamma^{\mu} T^{A} q_{r}\right)\left(\bar{u}_{s} \gamma^{\nu} T^{A} u_{t}\right) \widetilde{B}_{\mu \nu}$ \\
\hline$Q_{q^{2} d^{2} G}^{(1)}$ & $\left(\bar{q}_{p} \gamma^{\mu} q_{r}\right)\left(\bar{d}_{s} \gamma^{\nu} T^{A} d_{t}\right) G_{\mu \nu}^{A}$ \\
\hline$Q_{q^{2} d^{2} G}^{(2)}$ & $\left(\bar{q}_{p} \gamma^{\mu} q_{r}\right)\left(\bar{d}_{s} \gamma^{\nu} T^{A} d_{t}\right) \widetilde{G}_{\mu \nu}^{A}$ \\
\hline$Q_{q^{2} d^{2} G}^{(3)}$ & $\left(\bar{q}_{p} \gamma^{\mu} T^{A} q_{r}\right)\left(\bar{d}_{s} \gamma^{\nu} d_{t}\right) G_{\mu \nu}^{A}$ \\
\hline$Q_{q^{2} d^{2} G}^{(4)}$ & $\left(\bar{q}_{p} \gamma^{\mu} T^{A} q_{r}\right)\left(\bar{d}_{s} \gamma^{\nu} d_{t}\right) \widetilde{G}_{\mu \nu}^{A}$ \\
\hline$Q_{q^{2} d^{2} G}^{(5)}$ & $f^{A B C}\left(\bar{q}_{p} \gamma^{\mu} T^{A} q_{r}\right)\left(\bar{d}_{s} \gamma^{\nu} T^{B} d_{t}\right) G_{\mu \nu}^{C}$ \\
\hline$Q_{q^{2} d^{2} G}^{(6)}$ & $f^{A B C}\left(\bar{q}_{p} \gamma^{\mu} T^{A} q_{r}\right)\left(\bar{d}_{s} \gamma^{\nu} T^{B} d_{t}\right) \widetilde{G}_{\mu \nu}^{C}$ \\
\hline$Q_{q^{2} d^{2} G}^{(7)}$ & $d^{A B C}\left(\bar{q}_{p} \gamma^{\mu} T^{A} q_{r}\right)\left(\bar{d}_{s} \gamma^{\nu} T^{B} d_{t}\right) G_{\mu \nu}^{C}$ \\
\hline$Q_{q^{2} d^{2} G}^{(8)}$ & $d^{A B C}\left(\bar{q}_{p} \gamma^{\mu} T^{A} q_{r}\right)\left(\bar{d}_{s} \gamma^{\nu} T^{B} d_{t}\right) \widetilde{G}_{\mu \nu}^{C}$ \\
\hline$Q_{q^{2} d^{2} W}^{(1)}$ & $\left(\bar{q}_{p} \gamma^{\mu} \tau^{I} q_{r}\right)\left(\bar{d}_{s} \gamma^{\nu} d_{t}\right) W_{\mu \nu}^{I}$ \\
\hline$Q_{q^{2} d^{2} W}^{(2)}$ & $\left(\bar{q}_{p} \gamma^{\mu} \tau^{I} q_{r}\right)\left(\bar{d}_{s} \gamma^{\nu} d_{t}\right) \widetilde{W}_{\mu \nu}^{I}$ \\
\hline$Q_{q^{2} d^{2} W}^{(3)}$ & $\left(\bar{q}_{p} \gamma^{\mu} T^{A} \tau^{I} q_{r}\right)\left(\bar{d}_{s} \gamma^{\nu} T^{A} d_{t}\right) W_{\mu \nu}^{I}$ \\
\hline$Q_{q^{2} d^{2} W}^{(4)}$ & $\left(\bar{q}_{p} \gamma^{\mu} T^{A} \tau^{I} q_{r}\right)\left(\bar{d}_{s} \gamma^{\nu} T^{A} d_{t}\right) \widetilde{W}_{\mu \nu}^{I}$ \\
\hline$Q_{q^{2} d^{2} B}^{(1)}$ & $\left(\bar{q}_{p} \gamma^{\mu} q_{r}\right)\left(\bar{d}_{s} \gamma^{\nu} d_{t}\right) B_{\mu \nu}$ \\
\hline$Q_{q^{2} d^{2} B}^{(2)}$ & $\left(\bar{q}_{p} \gamma^{\mu} q_{r}\right)\left(\bar{d}_{s} \gamma^{\nu} d_{t}\right) \widetilde{B}_{\mu \nu}$ \\
\hline$Q_{q^{2} d^{2} B}^{(3)}$ & $\left(\bar{q}_{p} \gamma^{\mu} T^{A} q_{r}\right)\left(\bar{d}_{s} \gamma^{\nu} T^{A} d_{t}\right) B_{\mu \nu}$ \\
\hline$Q_{q^{2} d^{2} B}^{(4)}$ & $\left(\bar{q}_{p} \gamma^{\mu} T^{A} q_{r}\right)\left(\bar{d}_{s} \gamma^{\nu} T^{A} d_{t}\right) \widetilde{B}_{\mu \nu}$ \\
\hline
\end{tabular}

Table 13. The dimension-eight operators in the SMEFT of class-19 with field content $(\bar{L} L)(\bar{R} R) X$. The subscripts $p, r, s, t$ are weak-eigenstate indices. 


\begin{tabular}{|l|l|}
\hline \multicolumn{2}{|c|}{$19:(\overline{\boldsymbol{L}} \boldsymbol{R})(\overline{\boldsymbol{R}} \boldsymbol{L}) \boldsymbol{X}+$ h.c. } \\
\hline$Q_{\text {ledqG }}^{(1)}$ & $\left(\bar{l}_{p}^{j} \sigma^{\mu \nu} e_{r}\right)\left(\bar{d}_{s} T^{A} q_{t j}\right) G_{\mu \nu}^{A}$ \\
$Q_{\text {ledq } G}^{(2)}$ & $\left(\bar{l}_{p}^{j} e_{r}\right)\left(\bar{d}_{s} \sigma^{\mu \nu} T^{A} q_{t j}\right) G_{\mu \nu}^{A}$ \\
$Q_{\text {ledq } W}^{(1)}$ & $\left(\bar{l}_{p} \sigma^{\mu \nu} e_{r}\right) \tau^{I}\left(\bar{d}_{s} q_{t}\right) W_{\mu \nu}^{I}$ \\
$Q_{\text {ledqW }}^{(2)}$ & $\left(\bar{l}_{p} e_{r}\right) \tau^{I}\left(\bar{d}_{s} \sigma^{\mu \nu} q_{t}\right) W_{\mu \nu}^{I}$ \\
$Q_{\text {ledqB}}^{(1)}$ & $\left(\bar{l}_{p}^{j} \sigma^{\mu \nu} e_{r}\right)\left(\bar{d}_{s} q_{t j}\right) B_{\mu \nu}$ \\
$Q_{\text {ledqB }}^{(2)}$ & $\left(\bar{l}_{p}^{j} e_{r}\right)\left(\bar{d}_{s} \sigma^{\mu \nu} q_{t j}\right) B_{\mu \nu}$ \\
\hline
\end{tabular}

\begin{tabular}{|c|c|}
\hline \multicolumn{2}{|r|}{$19:(\bar{L} R)(\bar{L} R) X+$ h.c. } \\
\hline$Q_{q^{2} u d G}^{(1)}$ & $\left(\bar{q}_{p}^{j} \sigma^{\mu \nu} T^{A} u_{r}\right) \epsilon_{j k}\left(\bar{q}_{s}^{k} d_{t}\right) G_{\mu \nu}^{A}$ \\
\hline$Q_{q^{2} u d G}^{(2)}$ & $\left(\bar{q}_{p}^{j} \sigma^{\mu \nu} u_{r}\right) \epsilon_{j k}\left(\bar{q}_{s}^{k} T^{A} d_{t}\right) G_{\mu \nu}^{A}$ \\
\hline$Q_{q^{2} u d G}^{(3)}$ & $\left(\bar{q}_{p}^{j} T^{A} u_{r}\right) \epsilon_{j k}\left(\bar{q}_{s}^{k} \sigma^{\mu \nu} d_{t}\right) G_{\mu \nu}^{A}$ \\
\hline$Q_{q^{2} u d G}^{(4)}$ & $\left(\bar{q}_{p}^{j} u_{r}\right) \epsilon_{j k}\left(\bar{q}_{s}^{k} \sigma^{\mu \nu} T^{A} d_{t}\right) G_{\mu \nu}^{A}$ \\
\hline$Q_{q^{2} u d G}^{(5)}$ & $\left(\bar{q}_{p}^{j} \sigma^{\mu \rho} T^{A} u_{r}\right) \epsilon_{j k}\left(\bar{q}_{s}^{k} \sigma_{\rho \nu} d_{t}\right) G_{\mu}^{A \nu}$ \\
\hline$Q_{q^{2} u d G}^{(6)}$ & $\left(\bar{q}_{p}^{j} \sigma^{\mu \rho} u_{r}\right) \epsilon_{j k}\left(\bar{q}_{s}^{k} \sigma_{\rho \nu} T^{A} d_{t}\right) G_{\mu}^{A \nu}$ \\
\hline$Q_{q^{2} u d W}^{(1)}$ & $\left(\bar{q}_{p}^{j} \sigma^{\mu \nu} u_{r}\right)\left(\tau^{I} \epsilon\right)_{j k}\left(\bar{q}_{s}^{k} d_{t}\right) W_{\mu \nu}^{I}$ \\
\hline$Q_{q^{2} u d W}^{(2)}$ & $\left(\bar{q}_{p}^{j} u_{r}\right)\left(\tau^{I} \epsilon\right)_{j k}\left(\bar{q}_{s}^{k} \sigma^{\mu \nu} d_{t}\right) W_{\mu \nu}^{I}$ \\
\hline$Q_{q^{2} u d W}^{(3)}$ & $\left(\bar{q}_{p}^{j} \sigma^{\mu \rho} u_{r}\right)\left(\tau^{I} \epsilon\right)_{j k}\left(\bar{q}_{s}^{k} \sigma_{\rho \nu} d_{t}\right) W_{\mu}^{I \nu}$ \\
\hline$Q_{q^{2} u d B}^{(1)}$ & $\left(\bar{q}_{p}^{j} \sigma^{\mu \nu} u_{r}\right) \epsilon_{j k}\left(\bar{q}_{s}^{k} d_{t}\right) B_{\mu \nu}$ \\
\hline$Q_{q^{2} u d B}^{(2)}$ & $\left(\bar{q}_{p}^{j} u_{r}\right) \epsilon_{j k}\left(\bar{q}_{s}^{k} \sigma^{\mu \nu} d_{t}\right) B_{\mu \nu}$ \\
\hline$Q_{q^{2} u d B}^{(3)}$ & $\left(\bar{q}_{p}^{j} \sigma^{\mu \rho} u_{r}\right) \epsilon_{j k}\left(\bar{q}_{s}^{k} \sigma_{\rho \nu} d_{t}\right) B_{\mu}^{\nu}$ \\
\hline$Q_{\text {lequG }}^{(1)}$ & $\left(\bar{l}_{p}^{j} \sigma^{\mu \nu} e_{r}\right) \epsilon_{j k}\left(\bar{q}_{s}^{k} T^{A} u_{t}\right) G_{\mu \nu}^{A}$ \\
\hline$Q_{\text {lequG }}^{(2)}$ & $\left(\bar{l}_{p}^{j} e_{r}\right) \epsilon_{j k}\left(\bar{q}_{s}^{k} \sigma^{\mu \nu} T^{A} u_{t}\right) G_{\mu \nu}^{A}$ \\
\hline$Q_{\text {lequG }}^{(3)}$ & $\left(\bar{l}_{p}^{j} \sigma^{\mu \rho} e_{r}\right) \epsilon_{j k}\left(\bar{q}_{s}^{k} \sigma_{\rho \nu} T^{A} u_{t}\right) G_{\mu}^{A \nu}$ \\
\hline$Q_{\text {lequW }}^{(1)}$ & $\left(\bar{l}_{p}^{j} \sigma^{\mu \nu} e_{r}\right)\left(\tau^{I} \epsilon\right)_{j k}\left(\bar{q}_{s}^{k} u_{t}\right) W_{\mu \nu}^{I}$ \\
\hline$Q_{l e q u W}^{(2)}$ & $\left(\bar{l}_{p}^{j} e_{r}\right)\left(\tau^{I} \epsilon\right)_{j k}\left(\bar{q}_{s}^{k} \sigma^{\mu \nu} u_{t}\right) W_{\mu \nu}^{I}$ \\
\hline$Q_{\text {lequW }}^{(3)}$ & $\left(\bar{l}_{p}^{j} \sigma^{\mu \rho} e_{r}\right)\left(\tau^{I} \epsilon\right)_{j k}\left(\bar{q}_{s}^{k} \sigma_{\rho \nu} u_{t}\right) W_{\mu}^{I \nu}$ \\
\hline$Q_{\text {lequB }}^{(1)}$ & $\left(\bar{l}_{p}^{j} \sigma^{\mu \nu} e_{r}\right) \epsilon_{j k}\left(\bar{q}_{s}^{k} u_{t}\right) B_{\mu \nu}$ \\
\hline$Q_{l e q u B}^{(2)}$ & $\left(\bar{l}_{p}^{j} e_{r}\right) \epsilon_{j k}\left(\bar{q}_{s}^{k} \sigma^{\mu \nu} u_{t}\right) B_{\mu \nu}$ \\
\hline$Q_{\text {lequB }}^{(3)}$ & $\left(\bar{l}_{p}^{j} \sigma^{\mu \rho} e_{r}\right) \epsilon_{j k}\left(\bar{q}_{s}^{k} \sigma_{\rho \nu} u_{t}\right) B_{\mu}^{\nu}$ \\
\hline
\end{tabular}

Table 14. The dimension-eight operators in the SMEFT of class-19 with field content $(\bar{L} R)(\bar{R} L) X$ or $(\bar{L} R)(\bar{L} R) X$. All of the operators have Hermitian conjugates. The subscripts $p, r, s, t$ are weakeigenstate indices.

where $F_{\mu \nu}$ is the photon field strength, $\alpha$ is the fine structure constant, and $M_{e}$ is the mass of the electron. The normalization is such that order one coefficients are generated when the electron is integrated out with $E \ll M_{e}$. 


\begin{tabular}{|c|c|}
\hline \multicolumn{2}{|r|}{$19(\not B): \psi^{4} X+$ h.c. } \\
\hline$Q_{\text {lqudG }}^{(1)}$ & $\left(T^{A}\right)_{\gamma}^{\delta} \epsilon_{\delta \alpha \beta} \epsilon_{j k}\left(d_{p}^{\alpha} C \sigma^{\mu \nu} u_{r}^{\beta}\right)\left(q_{s}^{j \gamma} C l_{t}^{k}\right) G_{\mu \nu}^{A}$ \\
\hline$Q_{l q u d G}^{(2)}$ & $\left(T^{A}\right)_{\gamma}^{\delta} \epsilon_{\delta \alpha \beta} \epsilon_{j k}\left(d_{p}^{\alpha} C u_{r}^{\beta}\right)\left(q_{s}^{j \gamma} C \sigma^{\mu \nu} l_{t}^{k}\right) G_{\mu \nu}^{A}$ \\
\hline$Q_{l q u d G}^{(3)}$ & $\left(T^{A}\right)_{(\alpha}^{\delta} \epsilon_{\beta) \gamma \delta} \epsilon_{j k}\left(d_{p}^{\alpha} C \sigma^{\mu \nu} u_{r}^{\beta}\right)\left(q_{s}^{j \gamma} C l_{t}^{k}\right) G_{\mu \nu}^{A}$ \\
\hline$Q_{\text {lqudG }}^{(4)}$ & $\left(T^{A}\right)_{(\alpha}^{\delta} \epsilon_{\beta) \gamma \delta} \epsilon_{j k}\left(d_{p}^{\alpha} C u_{r}^{\beta}\right)\left(q_{s}^{j \gamma} C \sigma^{\mu \nu} l_{t}^{k}\right) G_{\mu \nu}^{A}$ \\
\hline$Q_{l q u d W}^{(1)}$ & $\epsilon_{\alpha \beta \gamma}\left(\tau^{I} \epsilon\right)_{j k}\left(d_{p}^{\alpha} C \sigma^{\mu \nu} u_{r}^{\beta}\right)\left(q_{s}^{j \gamma} C l_{t}^{k}\right) W_{\mu \nu}^{I}$ \\
\hline$Q_{l q u d W}^{(2)}$ & $\epsilon_{\alpha \beta \gamma}\left(\tau^{I} \epsilon\right)_{j k}\left(d_{p}^{\alpha} C u_{r}^{\beta}\right)\left(q_{s}^{j \gamma} C \sigma^{\mu \nu} l_{t}^{k}\right) W_{\mu \nu}^{I}$ \\
\hline$Q_{l q u d B}^{(1)}$ & $\epsilon_{\alpha \beta \gamma} \epsilon_{j k}\left(d_{p}^{\alpha} C \sigma^{\mu \nu} u_{r}^{\beta}\right)\left(q_{s}^{j \gamma} C l_{t}^{k}\right) B_{\mu \nu}$ \\
\hline$Q_{l q u d B}^{(2)}$ & $\epsilon_{\alpha \beta \gamma} \epsilon_{j k}\left(d_{p}^{\alpha} C u_{r}^{\beta}\right)\left(q_{s}^{j \gamma} C \sigma^{\mu \nu} l_{t}^{k}\right) B_{\mu \nu}$ \\
\hline$Q_{e q^{2} u G}^{(1)}$ & $\left(T^{A}\right)_{\gamma}^{\delta} \epsilon_{\delta \alpha \beta} \epsilon_{j k}\left(q_{p}^{j \alpha} C \sigma^{\mu \nu} q_{r}^{k \beta}\right)\left(u_{s}^{\gamma} C e_{t}\right) G_{\mu \nu}^{A}$ \\
\hline$Q_{e q^{2} u G}^{(2)}$ & $\left(T^{A}\right)_{(\alpha}^{\delta} \epsilon_{\beta) \gamma \delta} \epsilon_{j k}\left(q_{p}^{j \alpha} C q_{r}^{k \beta}\right)\left(u_{s}^{\gamma} C \sigma^{\mu \nu} e_{t}\right) G_{\mu \nu}^{A}$ \\
\hline$Q_{e q^{2} u W}^{(1)}$ & $\epsilon_{\alpha \beta \gamma}\left(\tau^{I} \epsilon\right)_{j k}\left(q_{p}^{j \alpha} C \sigma^{\mu \nu} q_{r}^{k \beta}\right)\left(u_{s}^{\gamma} C e_{t}\right) W_{\mu \nu}^{I}$ \\
\hline$Q_{e q^{2} u B}^{(1)}$ & $\epsilon_{\alpha \beta \gamma} \epsilon_{j k}\left(q_{p}^{j \alpha} C q_{r}^{k \beta}\right)\left(u_{s}^{\gamma} C \sigma^{\mu \nu} e_{t}\right) B_{\mu \nu}$ \\
\hline$Q_{l q^{3} G}^{(1)}$ & $\left(T^{A}\right)_{\gamma}^{\delta} \epsilon_{\delta \alpha \beta} \epsilon_{m n} \epsilon_{j k}\left(q_{p}^{m \alpha} C \sigma^{\mu \nu} q_{r}^{j \beta}\right)\left(q_{s}^{k \gamma} C l_{t}^{n}\right) G_{\mu \nu}^{A}$ \\
\hline$Q_{l q^{3} G}^{(2)}$ & $\left(T^{A}\right)_{(\alpha}^{\delta} \epsilon_{\beta) \gamma \delta} \epsilon_{m n} \epsilon_{j k}\left(q_{p}^{m \alpha} C q_{r}^{j \beta}\right)\left(q_{s}^{k \gamma} C \sigma^{\mu \nu} l_{t}^{n}\right) G_{\mu \nu}^{A}$ \\
\hline$Q_{l q^{3} W}^{(1)}$ & $\epsilon_{\alpha \beta \gamma}\left(\tau^{I} \epsilon\right)_{m n} \epsilon_{j k}\left(q_{p}^{m \alpha} C q_{r}^{j \beta}\right)\left(q_{s}^{k \gamma} C \sigma^{\mu \nu} l_{t}^{n}\right) W_{\mu \nu}^{I}$ \\
\hline$Q_{l q^{3} W}^{(2)}$ & $\epsilon_{\alpha \beta \gamma}\left(\tau^{I} \epsilon\right)_{m j} \epsilon_{k n}\left(q_{p}^{m \alpha} C \sigma^{\mu \nu} q_{r}^{j \beta}\right)\left(q_{s}^{k \gamma} C l_{t}^{n}\right) W_{\mu \nu}^{I}$ \\
\hline$Q_{l q^{3} B}^{(1)}$ & $\epsilon_{\alpha \beta \gamma} \epsilon_{m n} \epsilon_{j k}\left(q_{p}^{m \alpha} C q_{r}^{j \beta}\right)\left(q_{s}^{k \gamma} C \sigma^{\mu \nu} l_{t}^{n}\right) B_{\mu \nu}$ \\
\hline$Q_{e u^{2} d G}^{(1)}$ & $\left(T^{A}\right)_{\gamma}^{\delta} \epsilon_{\delta \alpha \beta}\left(d_{p}^{\alpha} C \sigma^{\mu \nu} u_{r}^{\beta}\right)\left(u_{s}^{\gamma} C e_{t}\right) G_{\mu \nu}^{A}$ \\
\hline$Q_{e u^{2} d G}^{(2)}$ & $\left(T^{A}\right)_{\gamma}^{\delta} \epsilon_{\delta \alpha \beta}\left(u_{p}^{\alpha} C \sigma^{\mu \nu} u_{r}^{\beta}\right)\left(d_{s}^{\gamma} C e_{t}\right) G_{\mu \nu}^{A}$ \\
\hline$Q_{e u^{2} d G}^{(3)}$ & $\left(T^{A}\right)_{(\alpha}^{\delta} \epsilon_{\beta) \gamma \delta}\left(u_{p}^{\alpha} C u_{r}^{\beta}\right)\left(d_{s}^{\gamma} C \sigma^{\mu \nu} e_{t}\right) G_{\mu \nu}^{A}$ \\
\hline$Q_{e u^{2} d B}^{(1)}$ & $\epsilon_{\alpha \beta \gamma}\left(d_{p}^{\alpha} C \sigma^{\mu \nu} u_{r}^{\beta}\right)\left(u_{s}^{\gamma} C e_{t}\right) B_{\mu \nu}$ \\
\hline$Q_{\text {eu }}^{(2)}{ }^{2} d B$ & $\epsilon_{\alpha \beta \gamma}\left(u_{p}^{\alpha} C \sigma^{\mu \nu} u_{r}^{\beta}\right)\left(d_{s}^{\gamma} C e_{t}\right) B_{\mu \nu}$ \\
\hline$Q_{e q^{2} u W}^{(2)}$ & $\epsilon_{\alpha \beta \gamma}\left(\tau^{I} \epsilon\right)_{j k}\left(q_{p}^{j \alpha} C q_{r}^{k \beta}\right)\left(u_{s}^{\gamma} C \sigma^{\mu \nu} e_{t}\right) W_{\mu \nu}^{I}$ \\
\hline$Q_{e q^{2} u B}^{(2)}$ & $\epsilon_{\alpha \beta \gamma} \epsilon_{j k}\left(q_{p}^{j \alpha} C \sigma^{\mu \nu} q_{r}^{k \beta}\right)\left(u_{s}^{\gamma} C e_{t}\right) B_{\mu \nu}$ \\
\hline$Q_{l q^{3} G}^{(3)}$ & $\left(T^{A}\right)_{(\alpha}^{\delta} \epsilon_{\beta) \gamma \delta} \epsilon_{m n} \epsilon_{j k}\left(q_{p}^{m \alpha} C \sigma^{\mu \nu} q_{r}^{j \beta}\right)\left(q_{s}^{k \gamma} C l_{t}^{n}\right) G_{\mu \nu}^{A}$ \\
\hline$Q_{l q^{3} G}^{(4)}$ & $\left(T^{A}\right)_{\gamma}^{\delta} \epsilon_{\delta \alpha \beta} \epsilon_{m n} \epsilon_{j k}\left(q_{p}^{m \alpha} C q_{r}^{j \beta}\right)\left(q_{s}^{k \gamma} C \sigma^{\mu \nu} l_{t}^{n}\right) G_{\mu \nu}^{A}$ \\
\hline$Q_{l q^{3} W}^{(3)}$ & $\epsilon_{\alpha \beta \gamma} \epsilon_{m n}\left(\tau^{I} \epsilon\right)_{j k}\left(q_{p}^{m \alpha} C q_{r}^{j \beta}\right)\left(q_{s}^{k \gamma} C \sigma^{\mu \nu} l_{t}^{n}\right) W_{\mu \nu}^{I}$ \\
\hline$Q_{l q^{3} B}^{(2)}$ & $\epsilon_{\alpha \beta \gamma} \epsilon_{m n} \epsilon_{j k}\left(q_{p}^{m \alpha} C \sigma^{\mu \nu} q_{r}^{j \beta}\right)\left(q_{s}^{k \gamma} C l_{t}^{n}\right) B_{\mu \nu}$ \\
\hline
\end{tabular}

Table 15. The baryon number violating dimension-eight operators of class-19. All of the operators have Hermitian conjugates. The subscripts $p, r, s, t$ are weak-eigenstate indices. Operators below the dashed line vanish when there is only one generation of fermions. 


\begin{tabular}{|c|c|}
\hline \multicolumn{2}{|r|}{$20: \psi^{4} H D+$ h.c. } \\
\hline$Q_{l^{3} e H D}^{(1)}$ & $i\left(\bar{l}_{p} \gamma^{\mu} l_{r}\right)\left[\left(\bar{l}_{s} e_{t}\right) D_{\mu} H\right]$ \\
\hline$Q_{l^{3} e H D}^{(2)}$ & $i\left(\bar{l}_{p} \gamma^{\mu} \tau^{I} l_{r}\right)\left[\left(\bar{l}_{s} e_{t}\right) \tau^{I} D_{\mu} H\right]$ \\
\hline$Q_{l^{3} e H D}^{(3)}$ & $i\left(\bar{l}_{p} \gamma^{\mu} l_{r}\right)\left[\left(D_{\mu} \bar{l}_{s} e_{t}\right) H\right]$ \\
\hline$Q_{l e^{3} H D}^{(1)}$ & $i\left(\bar{e}_{p} \gamma^{\mu} e_{r}\right)\left[\left(\bar{l}_{s} D_{\mu} e_{t}\right) H\right]$ \\
\hline$Q_{l e q^{2} H D}^{(1)}$ & $i\left(\bar{q}_{p} \gamma^{\mu} q_{r}\right)\left[\left(\bar{l}_{s} e_{t}\right) D_{\mu} H\right]$ \\
\hline$Q_{l e q^{2} H D}^{(2)}$ & $i\left(\bar{l}_{p} \gamma^{\mu} q_{r}^{\alpha}\right)\left[\left(\bar{q}_{s \alpha} e_{t}\right) D_{\mu} H\right]$ \\
\hline$Q_{l e q^{2} H D}^{(3)}$ & $i\left(\bar{q}_{p} \gamma^{\mu} \tau^{I} q_{r}\right)\left[\left(\bar{l}_{s} e_{t}\right) \tau^{I} D_{\mu} H\right]$ \\
\hline$Q_{l e q^{2} H D}^{(4)}$ & $i\left(\bar{l}_{p} \gamma^{\mu} \tau^{I} q_{r}^{\alpha}\right)\left[\left(\bar{q}_{s \alpha} e_{t}\right) \tau^{I} D_{\mu} H\right]$ \\
\hline$Q_{l e q^{2} H D}^{(5)}$ & $i\left(\bar{q}_{p} \gamma^{\mu} q_{r}\right)\left[\left(\bar{l}_{s} D_{\mu} e_{t}\right) H\right]$ \\
\hline$Q_{l e q^{2} H D}^{(6)}$ & $i\left(\bar{q}_{p} \gamma^{\nu} \tau^{I} q_{r}\right)\left[\left(\bar{l}_{s} D_{\mu} e_{t}\right) \tau^{I} H\right]$ \\
\hline$Q_{l e u^{2} H D}^{(1)}$ & $i\left(\bar{u}_{p} \gamma^{\mu} u_{r}\right)\left[\left(\bar{l}_{s} e_{t}\right) D_{\mu} H\right]$ \\
\hline$Q_{l e u^{2} H D}^{(2)}$ & $i\left(\bar{u}_{p \alpha} \gamma^{\mu} e_{r}\right)\left[\left(\bar{l}_{s} u_{t}^{\alpha}\right) D_{\mu} H\right]$ \\
\hline$Q_{l e u^{2} H D}^{(3)}$ & $i\left(\bar{u}_{p} \gamma^{\mu} u_{r}\right)\left[\left(D_{\mu} \bar{l}_{s} e_{t}\right) H\right]$ \\
\hline$Q_{l e d^{2} H D}^{(1)}$ & $i\left(\bar{d}_{p} \gamma^{\mu} d_{r}\right)\left[\left(\bar{l}_{s} e_{t}\right) D_{\mu} H\right]$ \\
\hline$Q_{l e d^{2} H D}^{(2)}$ & $i\left(\bar{d}_{p \alpha} \gamma^{\mu} e_{r}\right)\left[\left(\bar{l}_{s} d_{t}^{\alpha}\right) D_{\mu} H\right]$ \\
\hline$Q_{l e d^{2} H D}^{(3)}$ & $i\left(\bar{d}_{p} \gamma^{\mu} d_{r}\right)\left[\left(D_{\mu} \bar{l}_{s} e_{t}\right) H\right]$ \\
\hline$Q_{\text {leudHD }}^{(1)}$ & $i \epsilon_{j k}\left(\bar{u}_{p} \gamma^{\mu} d_{r}\right)\left(\bar{e}_{s} l_{t}^{j}\right) D_{\mu} H^{k}$ \\
\hline$Q_{\text {leudHD }}^{(2)}$ & $i \epsilon_{j k}\left(\bar{e}_{p} \gamma^{\mu} d_{r}^{\alpha}\right)\left(\bar{u}_{s \alpha} l_{t}^{j}\right) D_{\mu} H^{k}$ \\
\hline$Q_{-}^{(3)}{ }_{\text {leudHD }}$ & $i \epsilon_{j k}\left(\bar{u}_{p} \gamma^{\mu} d_{r}\right)\left(\bar{e}_{s} D_{\mu} l_{t}^{j}\right) H^{k}$ \\
\hline$Q_{l e^{3} H D}^{(2)}$ & $i\left(\bar{e}_{p} \gamma^{\mu} e_{r}\right)\left[\left(\bar{l}_{s} e_{t}\right) D_{\mu} H\right]$ \\
\hline
\end{tabular}

\begin{tabular}{|c|c|}
\hline \multicolumn{2}{|r|}{$20: \psi^{4} H D+$ h.c. } \\
\hline$Q_{l^{2} q u H D}^{(1)}$ & $i\left(\bar{l}_{p} \gamma^{\mu} l_{r}\right)\left[\left(\bar{q}_{s} u_{t}\right) D_{\mu} \widetilde{H}\right]$ \\
\hline$Q_{l^{2} q u H D}^{(2)}$ & $i\left(\bar{q}_{p \alpha} \gamma^{\mu} l_{r}\right)\left[\left(\bar{l}_{s} u_{t}^{\alpha}\right) D_{\mu} \widetilde{H}\right]$ \\
\hline$Q_{l^{2} q u H D}^{(3)}$ & $i\left(\bar{l}_{p} \gamma^{\mu} \tau^{I} l_{r}\right)\left[\left(\bar{q}_{s} u_{t}\right) \tau^{I} D_{\mu} \widetilde{H}\right]$ \\
\hline$Q_{l^{2} q u H D}^{(4)}$ & $i\left(\bar{q}_{p \alpha} \gamma^{\mu} \tau^{I} l_{r}\right)\left[\left(\bar{l}_{s} u_{t}^{\alpha}\right) \tau^{I} D_{\mu} \widetilde{H}\right]$ \\
\hline$Q_{l^{2} q u H D}^{(5)}$ & $i\left(\bar{l}_{p} \gamma^{\mu} l_{r}\right)\left[\left(\bar{q}_{s} D_{\mu} u_{t}\right) \widetilde{H}\right]$ \\
\hline$Q_{l^{2} q u H D}^{(6)}$ & $i\left(\bar{l}_{p} \gamma^{\mu} \tau^{I} l_{r}\right)\left[\left(\bar{q}_{s} D_{\mu} u_{t}\right) \tau^{I} \widetilde{H}\right]$ \\
\hline$Q_{e^{2} q u H D}^{(1)}$ & $i\left(\bar{e}_{p} \gamma^{\mu} e_{r}\right)\left[\left(\bar{q}_{s} u_{t}\right) D_{\mu} \widetilde{H}\right]$ \\
\hline$Q_{e^{2} q u H D}^{(2)}$ & $i\left(\bar{e}_{p} \gamma^{\mu} u_{r}^{\alpha}\right)\left[\left(\bar{q}_{s \alpha} e_{t}\right) D_{\mu} \widetilde{H}\right]$ \\
\hline$Q_{e^{2} q u H D}^{(3)}$ & $i\left(\bar{e}_{p} \gamma^{\mu} e_{r}\right)\left[\left(D_{\mu} \bar{q}_{s} u_{t}\right) \widetilde{H}\right]$ \\
\hline$Q_{q^{3} u H D}^{(1)}$ & $i\left(\bar{q}_{p} \gamma^{\mu} q_{r}\right)\left[\left(\bar{q}_{s} u_{t}\right) D_{\mu} \widetilde{H}\right]$ \\
\hline$Q_{q^{3} u H D}^{(2)}$ & $i\left(\bar{q}_{p} \gamma^{\mu} \tau^{I} q_{r}\right)\left[\left(\bar{q}_{s} u_{t}\right) \tau^{I} D_{\mu} \widetilde{H}\right]$ \\
\hline$Q_{q^{3} u H D}^{(3)}$ & $i\left(\bar{q}_{p} \gamma^{\mu} T^{A} q_{r}\right)\left[\left(\bar{q}_{s} T^{A} u_{t}\right) D_{\mu} \widetilde{H}\right]$ \\
\hline$Q_{q^{3} u H D}^{(4)}$ & $i\left(\bar{q}_{p} \gamma^{\mu} T^{A} \tau^{I} q_{r}\right)\left[\left(\bar{q}_{s} T^{A} u_{t}\right) \tau^{I} D_{\mu} \widetilde{H}\right]$ \\
\hline$Q_{q^{3} u H D}^{(5)}$ & $i\left(\bar{q}_{p} \gamma^{\mu} q_{r}\right)\left[\left(D_{\mu} \bar{q}_{s} u_{t}\right) \widetilde{H}\right]$ \\
\hline$Q_{q^{3} u H D}^{(6)}$ & $i\left(\bar{q}_{p} \gamma^{\mu} \tau^{I} q_{r}\right)\left[\left(D_{\mu} \bar{q}_{s} u_{t}\right) \tau^{I} \widetilde{H}\right]$ \\
\hline$Q_{q u^{3} H D}^{(1)}$ & $i\left(\bar{u}_{p} \gamma^{\mu} u_{r}\right)\left[\left(\bar{q}_{s} u_{t}\right) D_{\mu} \widetilde{H}\right]$ \\
\hline$Q_{q u^{3} H D}^{(2)}$ & $i\left(\bar{u}_{p} \gamma^{\mu} T^{A} u_{r}\right)\left[\left(\bar{q}_{s} T^{A} u_{t}\right) D_{\mu} \widetilde{H}\right]$ \\
\hline$Q_{q u^{3} H D}^{(3)}$ & $i\left(\bar{u}_{p} \gamma^{\mu} u_{r}\right)\left[\left(\bar{q}_{s} D_{\mu} u_{t}\right) \widetilde{H}\right]$ \\
\hline$Q_{q u d^{2} H D}^{(1)}$ & $i\left(\bar{d}_{p} \gamma^{\mu} d_{r}\right)\left[\left(\bar{q}_{s} u_{t}\right) D_{\mu} \widetilde{H}\right]$ \\
\hline$Q_{q u d^{2} H D}^{(2)}$ & $i\left(\bar{d}_{p} \gamma^{\mu} u_{r}\right)\left[\left(\bar{q}_{s} d_{t}\right) D_{\mu} \widetilde{H}\right]$ \\
\hline$Q_{q u d^{2} H D}^{(3)}$ & $i\left(\bar{d}_{p} \gamma^{\mu} T^{A} d_{r}\right)\left[\left(\bar{q}_{s} T^{A} u_{t}\right) D_{\mu} \widetilde{H}\right]$ \\
\hline$Q_{q u d^{2} H D}^{(4)}$ & $i\left(\bar{d}_{p} \gamma^{\mu} T^{A} u_{r}\right)\left[\left(\bar{q}_{s} T^{A} d_{t}\right) D_{\mu} \widetilde{H}\right]$ \\
\hline$Q_{q u d^{2} H D}^{(5)}$ & $i\left(\bar{d}_{p} \gamma^{\mu} d_{r}\right)\left[\left(D_{\mu} \bar{q}_{s} u_{t}\right) \widetilde{H}\right]$ \\
\hline$Q_{q u d^{2} H D}^{(6)}$ & $i\left(\bar{d}_{p} \gamma^{\mu} T^{A} d_{r}\right)\left[\left(D_{\mu} \bar{q}_{s} T^{A} u_{t}\right) \widetilde{H}\right]$ \\
\hline
\end{tabular}

Table 16. The dimension-eight operators in the SMEFT of class-20 whose field content superficially includes either an electron-type or up-quark-type Yukawa interaction. All of the operators have Hermitian conjugates. The subscripts $p, r, s, t$ are weak-eigenstate indices. The operator below the dashed line is redundant when there is only one generation of fermions. 


\begin{tabular}{|c|c|c|c|}
\hline \multicolumn{2}{|r|}{$20: \psi^{4} H D+$ h.c. } & \multicolumn{2}{|r|}{$20(\not B): \psi^{4} H D+$ h.c. } \\
\hline$Q_{l^{2} q d H D}^{(1)}$ & $i\left(\bar{l}_{p} \gamma^{\mu} l_{r}\right)\left[\left(\bar{q}_{s} d_{t}\right) D_{\mu} H\right]$ & $Q_{l u^{2} d H D}^{(1)}$ & $i \epsilon_{\alpha \beta \gamma}\left[D_{\mu} H^{\dagger}\left(u_{p}^{\alpha} C \gamma^{\mu} l_{r}\right)\right]\left(u_{s}^{\beta} C d_{t}^{\gamma}\right)$ \\
\hline$Q_{l^{2} q d H D}^{(2)}$ & $i\left(\bar{q}_{p \alpha} \gamma^{\mu} l_{r}\right)\left[\left(\bar{l}_{s} d_{t}^{\alpha}\right) D_{\mu} H\right]$ & $Q_{l u^{2} d H D}^{(2)}$ & $i \epsilon_{\alpha \beta \gamma}\left[H^{\dagger}\left(u_{p}^{\alpha} C \gamma^{\mu} l_{r}\right)\right]\left(D_{\mu} u_{s}^{\beta} C d_{t}^{\gamma}\right)$ \\
\hline$Q_{l^{2} q d H D}^{(3)}$ & $i\left(\bar{l}_{p} \gamma^{\mu} \tau^{I} l_{r}\right)\left[\left(\bar{q}_{s} d_{t}\right) \tau^{I} D_{\mu} H\right]$ & $Q_{l u d^{2} H D}^{(1)}$ & $i \epsilon_{\alpha \beta \gamma} \epsilon_{j k}\left(d_{p}^{\alpha} C \gamma^{\mu} l_{r}^{j}\right)\left(d_{s}^{\beta} C u_{t}^{\gamma}\right) D_{\mu} H^{k}$ \\
\hline$Q_{l^{2} q d H D}^{(4)}$ & $i\left(\bar{q}_{p \alpha} \gamma^{\mu} \tau^{I} l_{r}\right)\left[\left(\bar{l}_{s} d_{t}^{\alpha}\right) \tau^{I} D_{\mu} H\right]$ & $Q_{l u d^{2} H D}^{(2)}$ & $i \epsilon_{\alpha \beta \gamma} \epsilon_{j k}\left(d_{p}^{\alpha} C \gamma^{\mu} l_{r}^{j}\right)\left(D_{\mu} d_{s}^{\beta} C u_{t}^{\gamma}\right) H^{k}$ \\
\hline$Q_{l^{2} q d H D}^{(5)}$ & $i\left(\bar{l}_{p} \gamma^{\mu} l_{r}\right)\left[\left(\bar{q}_{s} D_{\mu} d_{t}\right) H\right]$ & $Q_{l q^{2} u H D}^{(1)}$ & $i \epsilon_{\alpha \beta \gamma} \epsilon_{j n} \epsilon_{k m} D_{\mu} H^{n \dagger}\left(q_{p}^{m \alpha} C \gamma^{\mu} u_{r}^{\beta}\right)\left(q_{s}^{j \gamma} C l_{t}^{k}\right)$ \\
\hline$Q_{l^{2} q d H D}^{(6)}$ & $i\left(\bar{l}_{p} \gamma^{\mu} \tau^{I} l_{r}\right)\left[\left(\bar{q}_{s} D_{\mu} d_{t}\right) \tau^{I} H\right]$ & $Q_{l q^{2} u H D}^{(2)}$ & $i \epsilon_{\alpha \beta \gamma} \epsilon_{k n} \epsilon_{j m} D_{\mu} H^{n \dagger}\left(q_{p}^{m \alpha} C \gamma^{\mu} u_{r}^{\beta}\right)\left(q_{s}^{j \gamma} C l_{t}^{k}\right)$ \\
\hline$Q_{e^{2} q d H D}^{(1)}$ & $i\left(\bar{e}_{p} \gamma^{\mu} e_{r}\right)\left[\left(\bar{q}_{s} d_{t}\right) D_{\mu} H\right]$ & $Q_{l q^{2} u H D}^{(3)}$ & $i \epsilon_{\alpha \beta \gamma} \epsilon_{j n} \epsilon_{k m} H^{n \dagger}\left(q_{p}^{m \alpha} C \gamma^{\mu} u_{r}^{\beta}\right)\left(D_{\mu} q_{s}^{j \gamma} C l_{t}^{k}\right)$ \\
\hline$Q_{e^{2} q d H D}^{(2)}$ & $i\left(\bar{e}_{p} \gamma^{\mu} d_{r}^{\alpha}\right)\left[\left(\bar{q}_{s \alpha} e_{t}\right) D_{\mu} H\right]$ & $Q_{l q^{2} d H D}^{(1)}$ & $i \epsilon_{\alpha \beta \gamma} \epsilon_{j n} \epsilon_{k m}\left(q_{p}^{m \alpha} C \gamma^{\mu} d_{r}^{\beta}\right)\left(q_{s}^{j \gamma} C l_{t}^{k}\right) D_{\mu} H^{n}$ \\
\hline$Q_{e^{2} q d H D}^{(3)}$ & $i\left(\bar{e}_{p} \gamma^{\mu} e_{r}\right)\left[\left(D_{\mu} \bar{q}_{s} d_{t}\right) H\right]$ & $Q_{l q^{2} d H D}^{(2)}$ & $i \epsilon_{\alpha \beta \gamma} \epsilon_{k n} \epsilon_{j m}\left(q_{p}^{m \alpha} C \gamma^{\mu} d_{r}^{\beta}\right)\left(q_{s}^{j \gamma} C l_{t}^{k}\right) D_{\mu} H^{n}$ \\
\hline$Q_{q^{3} d H D}^{(1)}$ & $i\left(\bar{q}_{p} \gamma^{\mu} q_{r}\right)\left[\left(\bar{q}_{s} d_{t}\right) D_{\mu} H\right]$ & $Q_{l q^{2} d H D}^{(3)}$ & $i \epsilon_{\alpha \beta \gamma} \epsilon_{j n} \epsilon_{k m}\left(q_{p}^{m \alpha} C \gamma^{\mu} d_{r}^{\beta}\right)\left(D_{\mu} q_{s}^{j \gamma} C l_{t}^{k}\right) H^{n}$ \\
\hline$Q_{q^{3} d H D}^{(2)}$ & $i\left(\bar{q}_{p} \gamma^{\mu} \tau^{I} q_{r}\right)\left[\left(\bar{q}_{s} d_{t}\right) \tau^{I} D_{\mu} H\right]$ & $Q_{e q^{3} H D}$ & $i \epsilon_{\alpha \beta \gamma} \epsilon_{m n} \epsilon_{j k}\left(q_{p}^{j \alpha} C \gamma^{\mu} e_{r}\right)\left(D_{\mu} q_{s}^{k \beta} C q_{t}^{m \gamma}\right) H^{n}$ \\
\hline$Q_{q^{3} d H D}^{(3)}$ & $i\left(\bar{q}_{p} \gamma^{\mu} T^{A} q_{r}\right)\left[\left(\bar{q}_{s} T^{A} d_{t}\right) D_{\mu} H\right]$ & $Q_{e q u^{2} H D}^{(1)}$ & $i \epsilon_{\alpha \beta \gamma}\left[D_{\mu} H^{\dagger}\left(u_{p}^{\alpha} C \gamma^{\mu} q_{r}^{\beta}\right)\right]\left(u_{s}^{\gamma} C e_{t}\right)$ \\
\hline$Q_{q^{3} d H D}^{(4)}$ & $i\left(\bar{q}_{p} \gamma^{\mu} T^{A} \tau^{I} q_{r}\right)\left[\left(\bar{q}_{s} T^{A} d_{t}\right) \tau^{I} D_{\mu} H\right]$ & $Q_{e q u^{2} H D}^{(2)}$ & $i \epsilon_{\alpha \beta \gamma}\left[H^{\dagger}\left(u_{p}^{\alpha} C \gamma^{\mu} q_{r}^{\beta}\right)\right]\left(D_{\mu} u_{s}^{\gamma} C e_{t}\right)$ \\
\hline$Q_{q^{3} d H D}^{(5)}$ & $i\left(\bar{q}_{p} \gamma^{\mu} q_{r}\right)\left[\left(D_{\mu} \bar{q}_{s} d_{t}\right) H\right]$ & $Q_{\text {equdHD }}^{(1)}$ & $i \epsilon_{\alpha \beta \gamma} \epsilon_{j k}\left(q_{p}^{j \alpha} C \gamma^{\mu} u_{r}^{\beta}\right)\left(d_{s}^{\gamma} C e_{t}\right) D_{\mu} H^{k}$ \\
\hline$Q_{q^{3} d H D}^{(6)}$ & $i\left(\bar{q}_{p} \gamma^{\mu} \tau^{I} q_{r}\right)\left[\left(D_{\mu} \bar{q}_{s} d_{t}\right) \tau^{I} H\right]$ & $Q_{\text {equdHD }}^{(2)}$ & $i \epsilon_{\alpha \beta \gamma} \epsilon_{j k}\left(q_{p}^{j \alpha} C \gamma^{\mu} d_{r}^{\beta}\right)\left(u_{s}^{\gamma} C e_{t}\right) D_{\mu} H^{k}$ \\
\hline$Q_{q u^{2} d H D}^{(1)}$ & $i\left(\bar{u}_{p} \gamma^{\mu} u_{r}\right)\left[\left(\bar{q}_{s} d_{t}\right) D_{\mu} H\right]$ & $Q_{\text {equdHD }}^{(3)}$ & $i \epsilon_{\alpha \beta \gamma} \epsilon_{j k}\left(q_{p}^{j \alpha} C \gamma^{\mu} u_{r}^{\beta}\right)\left(d_{s}^{\gamma} C D_{\mu} e_{t}\right) H^{k}$ \\
\hline$Q_{q u^{2} d H D}^{(2)}$ & $i\left(\bar{u}_{p} \gamma^{\mu} d_{r}\right)\left[\left(\bar{q}_{s} u_{t}\right) D_{\mu} H\right]$ & & \\
\hline$Q_{q u^{2} d H D}^{(3)}$ & $i\left(\bar{u}_{p} \gamma^{\mu} T^{A} u_{r}\right)\left[\left(\bar{q}_{s} T^{A} d_{t}\right) D_{\mu} H\right]$ & & \\
\hline$Q_{q u^{2} d H D}^{(4)}$ & $i\left(\bar{u}_{p} \gamma^{\mu} T^{A} d_{r}\right)\left[\left(\bar{q}_{s} T^{A} u_{t}\right) D_{\mu} H\right]$ & & \\
\hline$Q_{q u^{2} d H D}^{(5)}$ & $i\left(\bar{u}_{p} \gamma^{\mu} u_{r}\right)\left[\left(D_{\mu} \bar{q}_{s} d_{t}\right) H\right]$ & & \\
\hline$Q_{q u^{2} d H D}^{(6)}$ & $i\left(\bar{u}_{p} \gamma^{\mu} T^{A} u_{r}\right)\left[\left(D_{\mu} \bar{q}_{s} T^{A} d_{t}\right) H\right]$ & & \\
\hline$Q_{q d^{3} H D}^{(1)}$ & $i\left(\bar{d}_{p} \gamma^{\mu} d_{r}\right)\left[\left(\bar{q}_{s} d_{t}\right) D_{\mu} H\right]$ & & \\
\hline$Q_{q d^{3} H D}^{(2)}$ & $i\left(\bar{d}_{p} \gamma^{\mu} T^{A} d_{r}\right)\left[\left(\bar{q}_{s} T^{A} d_{t}\right) D_{\mu} H\right]$ & & \\
\hline$Q_{q d^{3} H D}^{(3)}$ & $i\left(\bar{d}_{p} \gamma^{\mu} d_{r}\right)\left[\left(\bar{q}_{s} D_{\mu} d_{t}\right) H\right]$ & & \\
\hline
\end{tabular}

Table 17. The dimension-eight operators in the SMEFT of class-20 whose field content superficially includes a down-quark-type Yukawa interaction or is baryon number violating. All of the operators have Hermitian conjugates. The subscripts $p, r, s, t$ are weak-eigenstate indices.

On the other hand, for a less restrictive energy range, $E \ll \Lambda$, the SMEFT Wilson coefficients from table 2 appearing in eq. (5.1) are

$$
\begin{aligned}
& \mathscr{C}_{L b L}^{(1)}=\frac{90 M_{e}^{4}}{\Lambda^{4}} 16 \pi^{2}\left[\frac{1}{g_{2}^{4}}\left(c_{W^{4}}^{(1)}+c_{W^{4}}^{(3)}\right)+\frac{1}{g_{1}^{4}} c_{B^{4}}^{(1)}+\frac{1}{g_{2}^{2} g_{1}^{2}}\left(c_{W^{2} B^{2}}^{(1)}+c_{W^{2} B^{2}}^{(3)}\right)\right] \\
& \mathscr{C}_{L b L}^{(2)}=\frac{90 M_{e}^{4}}{\Lambda^{4}} 16 \pi^{2}\left[\frac{1}{g_{2}^{4}}\left(c_{W^{4}}^{(2)}+c_{W^{4}}^{(4)}\right)+\frac{1}{g_{1}^{4}} c_{B^{4}}^{(2)}+\frac{1}{g_{2}^{2} g_{1}^{2}}\left(c_{W^{2} B^{2}}^{(2)}+c_{W^{2} B^{2}}^{(4)}\right)\right] \\
& \widetilde{\mathscr{C}}_{L b L}=\frac{90 M_{e}^{4}}{\Lambda^{4}} 16 \pi^{2}\left[\frac{1}{g_{2}^{4}}\left(c_{W^{4}}^{(5)}+c_{W^{4}}^{(6)}\right)+\frac{1}{g_{1}^{4}} c_{B^{4}}^{(3)}+\frac{1}{g_{2}^{2} g_{1}^{2}}\left(c_{W^{2} B^{2}}^{(5)}+c_{W^{2} B^{2}}^{(6)}+c_{W^{2} B^{2}}^{(7)}\right)\right]
\end{aligned}
$$




\begin{tabular}{|c|c|}
\hline \multicolumn{2}{|c|}{$\mathbf{2 1}(\overline{\boldsymbol{L}} \boldsymbol{L})(\overline{\boldsymbol{L}} \boldsymbol{L}) \boldsymbol{D}^{\mathbf{2}}$} \\
\hline$Q_{l^{4} D^{2}}^{(1)}$ & $D^{\nu}\left(\bar{l}_{p} \gamma^{\mu} l_{r}\right) D_{\nu}\left(\bar{l}_{s} \gamma_{\mu} l_{t}\right)$ \\
$Q_{l^{4} D^{2}}^{(2)}$ & $\left(\bar{l}_{p} \gamma^{\mu} \overleftrightarrow{D}^{\nu} l_{r}\right)\left(\bar{l}_{s} \gamma_{\mu} \overleftrightarrow{D}_{\nu} l_{t}\right)$ \\
$Q_{q^{4} D^{2}}^{(1)}$ & $D^{\nu}\left(\bar{q}_{p} \gamma^{\mu} q_{r}\right) D_{\nu}\left(\bar{q}_{s} \gamma_{\mu} q_{t}\right)$ \\
$Q_{q^{4} D^{2}}^{(2)}$ & $\left(\bar{q}_{p} \gamma^{\mu} \overleftrightarrow{D}^{\nu} q_{r}\right)\left(\bar{q}_{s} \gamma_{\mu} \overleftrightarrow{D}_{\nu} q_{t}\right)$ \\
$Q_{q^{4} D^{2}}^{(3)}$ & $D^{\nu}\left(\bar{q}_{p} \gamma^{\mu} \tau^{I} q_{r}\right) D_{\nu}\left(\bar{q}_{s} \gamma_{\mu} \tau^{I} q_{t}\right)$ \\
$Q_{q^{4} D^{2}}^{(4)}$ & $\left(\bar{q}_{p} \gamma^{\mu} \overleftrightarrow{D}^{I \nu} q_{r}\right)\left(\bar{q}_{s} \gamma_{\mu} \overleftrightarrow{D}_{\nu}^{I} q_{t}\right)$ \\
$Q_{l^{2} q^{2} D^{2}}^{(1)}$ & $D^{\nu}\left(\bar{l}_{p} \gamma^{\mu} l_{r}\right) D_{\nu}\left(\bar{q}_{s} \gamma_{\mu} q_{t}\right)$ \\
$Q_{l^{2} q^{2} D^{2}}^{(2)}$ & $\left(\bar{l}_{p} \gamma^{\mu} \overleftrightarrow{D}^{\nu} l_{r}\right)\left(\bar{q}_{s} \gamma_{\mu} \overleftrightarrow{D}_{\nu} q_{t}\right)$ \\
$Q_{l^{2} q^{2} D^{2}}^{(3)}$ & $D^{\nu}\left(\bar{l}_{p} \gamma^{\mu} \tau^{I} l_{r}\right) D_{\nu}\left(\bar{q}_{s} \gamma_{\mu} \tau^{I} q_{t}\right)$ \\
$Q_{l^{2} q^{2} D^{2}}^{(4)}$ & $\left(\bar{l}_{p} \gamma^{\mu} \overleftrightarrow{D}^{I \nu} l_{r}\right)\left(\bar{q}_{s} \gamma_{\mu} \overleftrightarrow{D}_{\nu}^{I} q_{t}\right)$ \\
\hline
\end{tabular}

\begin{tabular}{|c|c|}
\hline \multicolumn{2}{|c|}{$\mathbf{2 1}(\overline{\boldsymbol{R}} \boldsymbol{R})(\overline{\boldsymbol{R}} \boldsymbol{R}) \boldsymbol{D}^{\mathbf{2}}$} \\
\hline$Q_{e^{4} D^{2}}$ & $D^{\nu}\left(\bar{e}_{p} \gamma^{\mu} e_{r}\right) D_{\nu}\left(\bar{e}_{s} \gamma_{\mu} e_{t}\right)$ \\
$Q_{u^{4} D^{2}}^{(1)}$ & $D^{\nu}\left(\bar{u}_{p} \gamma^{\mu} u_{r}\right) D_{\nu}\left(\bar{u}_{s} \gamma_{\mu} u_{t}\right)$ \\
$Q_{u^{4} D^{2}}^{(2)}$ & $\left(\bar{u}_{p} \gamma^{\mu} \overleftrightarrow{D}^{\nu} u_{r}\right)\left(\bar{u}_{s} \gamma_{\mu} \overleftrightarrow{D}_{\nu} u_{t}\right)$ \\
$Q_{d^{4} D^{2}}^{(1)}$ & $D^{\nu}\left(\bar{d}_{p} \gamma^{\mu} d_{r}\right) D_{\nu}\left(\bar{d}_{s} \gamma_{\mu} d_{t}\right)$ \\
$Q_{d^{4} D^{2}}^{(2)}$ & $\left(\bar{d}_{p} \gamma^{\mu} \overleftrightarrow{D}^{\nu} d_{r}\right)\left(\bar{d}_{s} \gamma_{\mu} \overleftrightarrow{D}_{\nu} d_{t}\right)$ \\
$Q_{e^{2} u^{2} D^{2}}^{(1)}$ & $D^{\nu}\left(\bar{e}_{p} \gamma^{\mu} e_{r}\right) D_{\nu}\left(\bar{u}_{s} \gamma_{\mu} u_{t}\right)$ \\
$Q_{e^{2} u^{2} D^{2}}^{(2)}$ & $\left(\bar{e}_{p} \gamma^{\mu} \overleftrightarrow{D}^{\nu} e_{r}\right)\left(\bar{u}_{s} \gamma_{\mu} \overleftrightarrow{D}_{\nu} u_{t}\right)$ \\
$Q_{e^{2} d^{2} D^{2}}^{(1)}$ & $D^{\nu}\left(\bar{e}_{p} \gamma^{\mu} e_{r}\right) D_{\nu}\left(\bar{d}_{s} \gamma_{\mu} d_{t}\right)$ \\
$Q_{e^{2} d^{2} D^{2}}^{(2)}$ & $\left(\bar{e}_{p} \gamma^{\mu} \overleftrightarrow{D^{\nu}} e_{r}\right)\left(\bar{d}_{s} \gamma_{\mu} \overleftrightarrow{D}_{\nu} d_{t}\right)$ \\
$Q_{u^{2} d^{2} D^{2}}^{(1)}$ & $D^{\nu}\left(\bar{u}_{p} \gamma^{\mu} u_{r}\right) D_{\nu}\left(\bar{d}_{s} \gamma_{\mu} d_{t}\right)$ \\
$Q_{u^{2} d^{2} D^{2}}^{(2)}$ & $\left(\bar{u}_{p} \gamma^{\mu} \overleftrightarrow{D}^{\nu} u_{r}\right)\left(\bar{d}_{s} \gamma_{\mu} \overleftrightarrow{D}_{\nu} d_{t}\right)$ \\
$Q_{u^{2} d^{2} D^{2}}^{(3)}$ & $D^{\nu}\left(\bar{u}_{p} \gamma^{\mu} T^{A} u_{r}\right) D_{\nu}\left(\bar{d}_{s} \gamma_{\mu} T^{A} d_{t}\right)$ \\
$Q_{u^{2}}^{(4)} d^{2} D^{2}$ & $\left(\bar{u}_{p} \gamma^{\mu} T^{A} \overleftrightarrow{D^{\nu}} u_{r}\right)\left(\bar{d}_{s} \gamma_{\mu} T^{A} \overleftrightarrow{D}_{\nu} d_{t}\right)$ \\
\hline$Q_{e^{4} D^{2}}^{(2)}$ & $\left.-\underline{e}_{p} \gamma^{\mu} \overleftrightarrow{D}^{\nu} e_{r}\right)\left(\bar{e}_{s} \gamma_{\mu} \overleftrightarrow{D}_{\nu} e_{t}\right)$ \\
\hline
\end{tabular}

\begin{tabular}{|c|c|}
\hline \multicolumn{2}{|c|}{$\mathbf{2 1}:(\overline{\boldsymbol{L}} \boldsymbol{L})(\overline{\boldsymbol{R}} \boldsymbol{R}) \boldsymbol{D}^{\mathbf{2}}$} \\
\hline$Q_{l^{2} e^{2} D^{2}}^{(1)}$ & $D^{\nu}\left(\bar{l}_{p} \gamma^{\mu} l_{r}\right) D_{\nu}\left(\bar{e}_{s} \gamma_{\mu} e_{t}\right)$ \\
$Q_{l^{2} e^{2} D^{2}}$ & $\left(\bar{l}_{p} \gamma^{\mu} \overleftrightarrow{D}^{\nu} l_{r}\right)\left(\bar{e}_{s} \gamma_{\mu} \overleftrightarrow{D}_{\nu} e_{t}\right)$ \\
$Q_{l^{2} u^{2} D^{2}}^{(1)}$ & $D^{\nu}\left(\bar{l}_{p} \gamma^{\mu} l_{r}\right) D_{\nu}\left(\bar{u}_{s} \gamma_{\mu} u_{t}\right)$ \\
$Q_{l^{2} u^{2} D^{2}}^{(2)}$ & $\left(\bar{l}_{p} \gamma^{\mu} \overleftrightarrow{D^{\nu}} l_{r}\right)\left(\bar{u}_{s} \gamma_{\mu} \overleftrightarrow{D}_{\nu} u_{t}\right)$ \\
$Q_{l^{2} d^{2} D^{2}}^{(1)}$ & $D^{\nu}\left(\bar{l}_{p} \gamma^{\mu} l_{r}\right) D_{\nu}\left(\bar{d}_{s} \gamma_{\mu} d_{t}\right)$ \\
$Q_{l^{2} d^{2} D^{2}}^{(2)}$ & $\left(\bar{l}_{p} \gamma^{\mu} \overleftrightarrow{D}^{\nu} l_{r}\right)\left(\bar{d}_{s} \gamma_{\mu} \overleftrightarrow{D}_{\nu} d_{t}\right)$ \\
$Q_{q^{2} e^{2} D^{2}}^{(1)}$ & $D^{\nu}\left(\bar{q}_{p} \gamma^{\mu} q_{r}\right) D_{\nu}\left(\bar{e}_{s} \gamma_{\mu} e_{t}\right)$ \\
$Q_{q^{2} e^{2} D^{2}}^{(2)}$ & $\left(\bar{q}_{p} \gamma^{\mu} \overleftrightarrow{D}^{\nu} q_{r}\right)\left(\bar{e}_{s} \gamma_{\mu} \overleftrightarrow{D}_{\nu} e_{t}\right)$ \\
$Q_{q^{2} u^{2} D^{2}}^{(1)}$ & $D^{\nu}\left(\bar{q}_{p} \gamma^{\mu} q_{r}\right) D_{\nu}\left(\bar{u}_{s} \gamma_{\mu} u_{t}\right)$ \\
$Q_{q^{2} u^{2} D^{2}}^{(2)}$ & $\left(\bar{q}_{p} \gamma^{\mu} \overleftrightarrow{D}^{\nu} q_{r}\right)\left(\bar{u}_{s} \gamma_{\mu} \overleftrightarrow{D}_{\nu} u_{t}\right)$ \\
$Q_{q^{2} u^{2} D^{2}}^{(3)}$ & $D^{\nu}\left(\bar{q}_{p} \gamma^{\mu} T^{A} q_{r}\right) D_{\nu}\left(\bar{u}_{s} \gamma_{\mu} T^{A} u_{t}\right)$ \\
$Q_{q^{2} u^{2} D^{2}}^{(4)}$ & $\left(\bar{q}_{p} \gamma^{\mu} T^{A} \overleftrightarrow{D}^{\nu} q_{r}\right)\left(\bar{u}_{s} \gamma_{\mu} T^{A} \overleftrightarrow{D}_{\nu} u_{t}\right)$ \\
$Q_{q^{2} d^{2} D^{2}}^{(1)}$ & $D^{\nu}\left(\bar{q}_{p} \gamma^{\mu} q_{r}\right) D_{\nu}\left(\bar{d}_{s} \gamma_{\mu} d_{t}\right)$ \\
$Q_{q^{2} d^{2} D^{2}}^{(2)}$ & $\left(\bar{q}_{p} \gamma^{\mu} \overleftrightarrow{D}^{\nu} q_{r}\right)\left(\bar{d}_{s} \gamma_{\mu} \overleftrightarrow{D}_{\nu} d_{t}\right)$ \\
$Q_{q^{2} d^{2} D^{2}}^{(3)}$ & $D^{\nu}\left(\bar{q}_{p} \gamma^{\mu} T^{A} q_{r}\right) D_{\nu}\left(\bar{d}_{s} \gamma_{\mu} T^{A} d_{t}\right)$ \\
$Q_{q^{2} d^{2} D^{2}}^{(4)}$ & $\left(\bar{q}_{p} \gamma^{\mu} T^{A} \overleftrightarrow{D}^{\nu} q_{r}\right)\left(\bar{d}_{s} \gamma_{\mu} T^{A} \overleftrightarrow{D}_{\nu} d_{t}\right)$ \\
\hline
\end{tabular}

\begin{tabular}{|c|c|}
\hline \multicolumn{2}{|c|}{$21:(\overline{\boldsymbol{L}} \boldsymbol{R})(\overline{\boldsymbol{L}} \boldsymbol{R}) \boldsymbol{D}^{\mathbf{2}}+$ h.c. } \\
\hline$Q_{q^{2} u d D^{2}}^{(1)}$ & $D_{\mu}\left(\bar{q}_{p}^{j} u_{r}\right) \epsilon_{j k} D^{\mu}\left(\bar{q}_{s}^{k} d_{t}\right)$ \\
$Q_{q^{2} u d D^{2}}^{(2)}$ & $D_{\mu}\left(\bar{q}_{p}^{j} T^{A} u_{r}\right) \epsilon_{j k} D^{\mu}\left(\bar{q}_{s}^{k} T^{A} d_{t}\right)$ \\
$Q_{q^{2} u d D^{2}}^{(3)}$ & $\left(\bar{q}_{p}^{j} \overleftrightarrow{D}^{\mu} u_{r}\right) \epsilon_{j k}\left(\bar{q}_{s}^{k} \overleftrightarrow{D}_{\mu} d_{t}\right)$ \\
$Q_{\text {lequD }}^{(1)}$ & $D_{\mu}\left(\bar{l}_{p}^{j} e_{r}\right) \epsilon_{j k} D^{\mu}\left(\bar{q}_{s}^{k} u_{t}\right)$ \\
$Q_{\text {lequD }}^{(2)}$ & $D_{\mu}\left(\bar{l}_{p}^{j} u_{r}^{\alpha}\right) \epsilon_{j k} D^{\mu}\left(\bar{q}_{s \alpha}^{k} e_{t}\right)$ \\
$Q_{\text {lequD }}^{(3)}$ & $\left(\bar{l}_{p}^{j} \overleftrightarrow{D}^{\mu} e_{r}\right) \epsilon_{j k}\left(\bar{q}_{s}^{k} \overleftrightarrow{D}_{\mu} u_{t}\right)$ \\
\hline
\end{tabular}

Table 18. Most of the dimension-eight operators in the SMEFT of class-21, which are further divided into subclasses according to their chiral properties. See table 10 for the remaining class-21 operators. Operators with + h.c. have Hermitian conjugates. The subscripts $p, r, s, t$ are weakeigenstate indices. 
where we have dropped terms that are not enhanced by $\mathcal{O}\left(16 \pi^{2}\right)$. If the physics beyond the SM (BSM) that generates (5.2) is loop suppressed then these additional terms must be included.

Axiomatic principles of quantum field theory such as unitarity, analyticity, and crossing symmetry yield constraints on the parameters of a theory, see e.g. [36, 37]. For example, refs. [38, 39] used a once-subtracted dispersion relation to derive sum rules for the couplings of an extended Higgs boson sector. Dimension-8 SMEFT operators with four derivatives are constrained by twice-subtracted dispersion relations, which lead to positivity bounds on Wilson coefficients [20, 25, 40-42]. This follows from the contour at infinity vanishing due to the Froissart bound [43], and the crossed-channel branch-cut having the same sign the original-channel making the sum of non-vanishing integrals positive-definite by the optical theorem.

The positivity bounds on the coefficients in (5.2) are [20]

$$
\begin{aligned}
\mathscr{C}_{L b L}^{(1)} & >0, \\
\mathscr{C}_{L b L}^{(2)} & >0, \\
4 \mathscr{C}_{L b L}^{(1)} \mathscr{C}_{L b L}^{(2)} & >\left(\widetilde{\mathscr{C}}_{L b L}\right)^{2} .
\end{aligned}
$$

Unsurprisingly QED satisfies these bounds, $\mathscr{C}_{L b L}^{(1)}=1$ and $\mathscr{C}_{L b L}^{(2)}=7 / 4$ when $E \ll M_{e}$ and the electron is integrated out at one-loop [44]. The coefficient $\widetilde{\mathscr{C}}_{L b L}$ is not generated in QED as electromagnetic interactions conserve parity, which also satisfies (5.3). The bounds 5.3 can be combined with other positivity bounds coming from different initial scattering states to further constrain the $X^{4}$ SMEFT Wilson coefficients.

\subsection{Electroweak precision data}

Historically, the constraints on BSM physics from electroweak precision data were frequently summarized in terms of the parameters $S, T$, and $U$ [45-50]. The leading contributions to $S$ and $T$ come from dimension-6 operators, whereas $U$ first arises from a dimension-8 operator [21] motivating its discussion here. Additionally this discussion will help frame the results of section 5.3. However, for heavy BSM physics, it is important to keep in mind that the SMEFT is the preferred framework to use to describe electroweak precision data as it is completely general, unlike an $S T U$ analysis. For example, the dimension-6 operators that contribute to $S$ and $T$ also contribute to Higgs and diboson processes, see e.g. [51]. To put a modern twist on this analysis we use the geometric interpretation of the SMEFT (geoSMEFT) [52-54] to formulate expressions for $S, T$, and $U$ to all orders in $v_{T} / \Lambda$, with $v_{T}$ defined in eq. (5.5).

Higher-dimensional operators change the definitions of SM parameters in a variety of ways. Field redefinitions are needed to relate these combinations of inputs to measured quantities. To start, consider the potential for the Higgs field in the SMEFT through dimension-8

$$
V_{\mathrm{SMEFT}}=\lambda\left(H^{\dagger} H-\frac{v^{2}}{2}\right)^{2}-\frac{c_{H}}{\Lambda^{2}}\left(H^{\dagger} H\right)^{3}-\frac{c_{H^{8}}}{\Lambda^{4}}\left(H^{\dagger} H\right)^{4}
$$


The dimension-8 operators relevant for EWPD are given in tables 2 and 3. Due to the presence of the higher-dimensional operators in eq. (5.4), the minimum of Higgs boson potential is shifted $[19,53]$

$$
\left\langle H^{\dagger} H\right\rangle \equiv \frac{v_{T}^{2}}{2}=\frac{v^{2}}{2}\left(1+\frac{v^{2}}{\Lambda^{2}} \frac{3 c_{H}}{4 \lambda}+\frac{v^{4}}{\Lambda^{4}} \frac{9 c_{H}^{2}+4 \lambda c_{H^{8}}}{8 \lambda^{2}}\right)
$$

with $v_{T} \approx 246 \mathrm{GeV}$.

Now we turn to canonically normalizing the electroweak gauge, Higgs, and Goldstone bosons. Care must be taken as the field redefinitions are matrix equations. The geometric interpretation provides an elegant way to perform these transformations to all-orders in $v_{T} / \Lambda$. The Higgs-derivative operators through dimension- 8 are

$$
\begin{aligned}
\mathcal{L}_{H, \text { kin }}= & \left(D_{\mu} H^{\dagger}\right)\left(D^{\mu} H\right)+\frac{c_{H \square}}{\Lambda^{2}}\left(H^{\dagger} H\right) \square\left(H^{\dagger} H\right)+\frac{c_{H D}}{\Lambda^{2}}\left[\left(D_{\mu} H^{\dagger}\right) H\right]\left[H^{\dagger}\left(D^{\mu} H\right)\right] \\
& +\frac{c_{H^{6} D^{2}}^{(1)}}{\Lambda^{4}}\left(H^{\dagger} H\right)^{2}\left(D_{\mu} H^{\dagger} D^{\mu} H\right)+\frac{c_{H^{6} D^{2}}^{(2)}}{\Lambda^{4}}\left(H^{\dagger} H\right)\left(H^{\dagger} \tau^{I} H\right)\left(D_{\mu} H^{\dagger} \tau^{I} D^{\mu} H\right) .
\end{aligned}
$$

Defining

$$
H=\frac{1}{\sqrt{2}}\left(\begin{array}{l}
\phi_{2}+i \phi_{1} \\
\phi_{4}-i \phi_{3}
\end{array}\right),
$$

we can write eq. (5.6) in the language of the geoSMEFT as

$$
\mathcal{L}_{H, \mathrm{kin}}=\frac{1}{2} h_{\mathcal{I} \mathcal{J}}(\phi) \phi^{\mathcal{I}} \phi^{\mathcal{J}},
$$

where $\phi_{\mathcal{I}}=\left(\phi_{1}, \phi_{2}, \phi_{3}, \phi_{4}\right)$ with $\left\langle\phi_{\mathcal{I}}\right\rangle=v_{T} \delta_{\mathcal{I} 4}$. The weak eigenstates are related to the mass eigenstates through a metric on Higgs field space, $h_{\mathcal{I} \mathcal{J}}$, and a unitary matrix not considered here. We are working with the weak eigenstates and have not introduced the mass eigenstates here as all we need is the metric $h_{\mathcal{I} \mathcal{J}}$ to define the $T$ parameter. See ref. [53] for expressions involving mass eigenstates. The gauge kinetic terms to all-orders in $v_{T} / \Lambda$ are

$$
\begin{aligned}
\mathcal{L}_{\mathrm{EW}, \text { kin }}= & -\frac{1}{4} W_{\mu \nu}^{I} W^{I \mu \nu}-\frac{1}{4} B_{\mu \nu} B^{\mu \nu} \\
& +\frac{c_{H W}}{\Lambda^{2}}\left(H^{\dagger} H\right) W_{\mu \nu}^{I} W^{I \mu \nu}+\frac{c_{H B}}{\Lambda^{2}}\left(H^{\dagger} H\right) B_{\mu \nu} B^{\mu \nu}+\frac{c_{H W B}}{\Lambda^{2}}\left(H^{\dagger} \tau^{I} H\right) W_{\mu \nu}^{I} B^{\mu \nu} \\
& +\sum_{n=0}^{\infty} \frac{H^{2 n}}{\Lambda^{4+2 n}}\left(c_{B^{2} H^{4+2 n}}^{(1)}\left(H^{\dagger} H\right)^{2} B_{\mu \nu} B^{\mu \nu}+c_{W^{2} H^{4+2 n}}^{(1)}\left(H^{\dagger} H\right)^{2} W_{\mu \nu}^{I} W^{I \mu \nu}\right. \\
& \left.+c_{W B H^{4+2 n}}^{(1)}\left(H^{\dagger} H\right)\left(H^{\dagger} \tau^{I} H\right) W_{\mu \nu}^{I} B^{\mu \nu}+c_{W^{2} H^{4+2 n}}^{(3)}\left(H^{\dagger} \tau^{I} H\right)\left(H^{\dagger} \tau^{J} H\right) W_{\mu \nu}^{I} W^{J \mu \nu}\right)
\end{aligned}
$$

In the geoSMEFT eq. (5.9) takes the form

$$
\mathcal{L}_{\mathrm{EW}, \text { kin }}=-\frac{1}{4} g_{\mathcal{A B}}(H) \mathcal{W}^{\mathcal{A}} \mathcal{W}^{\mathcal{B}},
$$

where $\mathcal{W}^{\mathcal{A}}=\left(W^{I}, B\right)=\left(W^{1}, W^{2}, W^{3}, B\right)$ and $g_{\mathcal{A B}}$ is another metric on Higgs field space This metric, $g_{\mathcal{A B}}$, again along with a unitary matrix not considered here, relates the weak eigenstate gauge bosons to the mass eigenstates. 
The geometric definitions of $S, T$, and $U$ are

$$
\begin{aligned}
\frac{1}{16 \pi} S & =\left\langle g_{34}\right\rangle+\left\langle g_{43}\right\rangle, \\
\bar{\alpha} T & =\left\langle h_{11}\right\rangle-\left\langle h_{33}\right\rangle=\left\langle h_{22}\right\rangle-\left\langle h_{33}\right\rangle, \\
\frac{1}{16 \pi} U & =\left\langle g_{11}\right\rangle-\left\langle g_{33}\right\rangle=\left\langle g_{22}\right\rangle-\left\langle g_{33}\right\rangle
\end{aligned}
$$

where $\bar{\alpha}$ takes into account shift in the definition of the fine structure constant due to the presence of higher-dimensional operators, see appendix A of [54] for an explicit expression. Note this shift does not affect our analysis of light-by-light scattering; since all of those effects start at dimension- 8 we can freely trade $\alpha$ for $\bar{\alpha}$. Given (5.11) it straightforward to work out the contributions to $S, T$, and $U$

$$
\begin{aligned}
\frac{1}{16 \pi} S & =\frac{v_{T}^{2}}{\Lambda^{2}} c_{H W B}+\sum_{n=0}^{\infty} \frac{v_{T}^{4+2 n}}{2^{n} \Lambda^{4+2 n}} c_{W B H^{4+2 n}}^{(1)}, \\
\bar{\alpha} T & =-\frac{v_{T}^{2}}{2 \Lambda^{2}} c_{H D}-\frac{v_{T}^{4}}{2 \Lambda^{4}} c_{H^{6} D^{2}}^{(2)}, \\
\frac{1}{16 \pi} U & =\sum_{n=0}^{\infty} \frac{v_{T}^{4+2 n}}{2^{n} \Lambda^{4+2 n}} c_{W^{2} H^{4+2 n}}^{(3)},
\end{aligned}
$$

where we give $T$ to $\mathcal{O}\left(v_{T}^{4} / \Lambda^{4}\right)$ and $S$ and $U$ to all-orders in $v_{T} / \Lambda$.

\subsection{Scalar SU(2) $)_{w}$ quartets}

Although the dimension- 6 operators are formally the leading terms in the EFT expansion, there are various reasons why it may be necessary to consider dimension- 8 effects. Here we explore a scenario where the difference in experimental precision in different classes of measurements causes dimension- 8 effects to be important. The measurements are double Higgs boson production for which only upper limits exists on the cross section exist $[55,56]$, and EWPD, which as the name implies, is precisely measured. For example, one of the more precisely measured observables in this class is the width of the $Z$ boson, which has a relative precision of $9 \cdot 10^{-4}[57]$.

The models under consideration add to the SM field content a new scalar field, $\Theta$, that is an $\mathrm{SU}(2)_{w}$ quartet with either $\mathrm{y}=3 / 2$ or $1 / 2$. The Lagrangian for the $\mathrm{y}=3 / 2$ case is

$$
\begin{aligned}
\mathcal{L}_{\Theta}= & D_{\mu} \Theta^{\dagger} D^{\mu} \Theta-M^{2} \Theta^{\dagger} \Theta+\left[\lambda_{1} \Theta_{j k m}^{\dagger} H^{j} H^{k} H^{m}+\text { h.c. }\right] \\
& -\lambda_{\alpha 1}\left(H^{\dagger} H\right)\left(\Theta^{\dagger} \Theta\right)-\lambda_{\alpha 2}\left(H_{n}^{\dagger} H^{m}\right)\left(\Theta_{j k m}^{\dagger} \Theta^{j k n}\right)+\mathcal{O}\left(\Theta^{4}\right) .
\end{aligned}
$$

For the $\mathrm{y}=1 / 2$ case the term linear in $\Theta$ is instead $\left[\lambda_{1} \Theta_{j k m}^{\dagger} H^{j} H^{k} \widetilde{H}^{m}+\right.$ h.c. $]$. Note that $\Theta$ is completely symmetric in its $\mathrm{SU}(2)_{w}$ indices. Assuming $M \gg v_{T}$ we integrate out $\Theta$ and match to the SMEFT. In both cases only one dimension-6 operator, $\left(H^{\dagger} H\right)^{3}$, is generated at tree level $[22,58,59]$

$$
C_{H}=\frac{\left|\lambda_{1}\right|^{2}}{M^{2}}
$$


while at one-loop the triple $W$ operator, $Q_{W}$, is also generated $[58,60]$. At this stage the scalar quartets look like great candidates to enhance the double Higgs boson production rate.

However things are not so simple. When electroweak symmetry is broken the term linear in $\Theta$ in eq. (5.13) will force $\Theta$ to have a non-zero vacuum expectation value (vev). If $\Theta$ gets a vev, $v_{\Theta}$, its quantum numbers dictate that it contributes to the $T$ parameter [22]

$$
1+\bar{\alpha} T=\frac{v_{T}^{2}}{v_{T}^{2}-2 v_{\Theta}^{2}\left[\frac{3}{2}\left(\frac{3}{2}+1\right)-3 \mathrm{y}^{2}\right]}
$$

With this in mind we extend the matching to dimension- 8 starting with the $y=3 / 2$ case. We find

$$
\begin{aligned}
\mathcal{L}_{\Theta}^{(d=8)}= & -\frac{\lambda_{\alpha}\left|\lambda_{1}\right|^{2}}{M^{4}}\left(H^{\dagger} H\right)^{4}+\frac{3\left|\lambda_{1}\right|^{2}}{M^{4}}\left(H^{\dagger} H\right)^{2}\left(D_{\mu} H^{\dagger}\right)\left(D^{\mu} H\right) \\
& +\frac{6\left|\lambda_{1}\right|^{2}}{M^{4}}\left(H^{\dagger} H\right) H_{j}^{\dagger} H^{k}\left(D_{\mu} H_{k}^{\dagger}\right)\left(D^{\mu} H^{j}\right)
\end{aligned}
$$

with $\lambda_{\alpha}=\lambda_{\alpha 1}+\lambda_{\alpha 2}$. The last term on the right-hand side of eq. (5.16) is not in our operator basis. We use the Fierz identity for Pauli matrices, eq. (3.1), to convert that operator into our basis, yielding for the $\mathrm{y}=3 / 2$ case

$$
C_{H^{8}}=-\frac{\lambda_{\alpha}\left|\lambda_{1}\right|^{2}}{M^{4}}, \quad C_{H^{6} D^{2}}^{(1)}=\frac{6\left|\lambda_{1}\right|^{2}}{M^{4}}, \quad C_{H^{6} D^{2}}^{(2)}=\frac{3\left|\lambda_{1}\right|^{2}}{M^{4}}
$$

The $\mathrm{y}=1 / 2$ case is slightly more complicated

$$
\begin{aligned}
& \mathcal{L}_{\Theta}^{(d=8)}=-\frac{\lambda_{\alpha}\left|\lambda_{1}\right|^{2}}{M^{4}}\left(H^{\dagger} H\right)^{4}+\frac{3\left|\lambda_{1}\right|^{2}}{M^{4}}\left(H^{\dagger} H\right)^{2}\left(D_{\mu} H^{\dagger}\right)\left(D^{\mu} H\right) \\
&+\frac{2\left|\lambda_{1}\right|^{2}}{M^{4}}\left(H^{\dagger} H\right)\left[H_{j}^{\dagger} H^{k}\left(D_{\mu} H_{k}^{\dagger}\right)\left(D^{\mu} H^{j}\right)\right. \\
&\left.+H_{j}^{\dagger} H_{k}^{\dagger}\left(D_{\mu} H^{k}\right)\left(D^{\mu} H^{j}\right)+H^{j} H^{k}\left(D_{\mu} H_{k}^{\dagger}\right)\left(D^{\mu} H_{j}^{\dagger}\right)\right]
\end{aligned}
$$

Integrating the last two terms by parts, applying the Higgs boson EOM to fields with two derivatives acting on them, and using eq. (3.1) we find

$$
\begin{aligned}
& C_{H}=\left(1-\frac{2 \lambda v^{2}}{M^{2}}\right) \frac{\left|\lambda_{1}\right|^{2}}{M^{2}}, \quad C_{H^{8}}=-\left(\lambda_{\alpha}+4 \lambda\right) \frac{\left|\lambda_{1}\right|^{2}}{M^{4}}, \quad C_{H^{6} D^{2}}^{(2)}=-\frac{\left|\lambda_{1}\right|^{2}}{M^{4}} \\
& C_{l e H^{5}}=-Y_{e}^{\dagger} \frac{\left|\lambda_{1}\right|^{2}}{M^{4}}, \quad C_{q u H^{5}}=-Y_{u}^{\dagger} \frac{\left|\lambda_{1}\right|^{2}}{M^{4}}, \quad C_{q d H^{5}}=-Y_{d}^{\dagger} \frac{\left|\lambda_{1}\right|^{2}}{M^{4}} .
\end{aligned}
$$

We included the dimension- 6 coefficient $C_{H}$ here as it receives another contribution from mass term in the Higgs EOM, which was used to reduce a dimension- 8 operator into operators in our basis. This completes the matching of the scalar quartet models to the SMEFT at dimension-8.

Using (5.12) we see that $T_{\mathrm{y}=3 / 2}=-3 T_{\mathrm{y}=1 / 2}=-\frac{3}{2 \bar{\alpha}}\left|\lambda_{1}\right|^{2}\left(\frac{v_{T}}{M}\right)^{4}$. Our result for the contributions of these models to the $T$ parameter agrees with what was found in ref. [22]. The implication of our matching results is that these model cannot in fact provide a large enhancement to double Higgs boson production. This was unclear, from an EFT perspective at least, until the matching was done at dimension-8. 


\section{Comments on renormalization group evolution}

By construction measurements in an effective field theory take place at energies below the cutoff scale of the EFT. As such, to link measurements to parameters of possible physics in the UV, it is crucial to understand how the coefficients EFT operators evolve from one scale to another. This behavior is described by the renormalization group evolution equations

$$
\gamma_{i j} C_{j}=16 \pi^{2} \mu \frac{d C_{i}}{d \mu} \equiv \dot{C}_{i},
$$

where $\gamma$ is the anomalous dimension matrix and $\mu$ is the renormalization scale.

The renormalization group evolution of the SMEFT operators at one-loop is known at dimension-5 [61], dimension- 6 [5, 6, 26, 62], and dimension-7 [17, 63]. The loop diagrams in the preceding computations were built from one dimension- $d$ SMEFT contact amplitude and one tree level SM amplitude. For the dimension-6 RGE there are also one-loop amplitudes resulting from two insertions of dimension-5 operators, and ref. [64] computed these contributions to the RGE equations. Typically, the two dimension-5 operator contribution to the dimension- 6 RGE will be suppressed with respect to the one dimension- 6 contribution because all odd mass dimension operators in the SMEFT violate at least one of baryon and lepton number $[65,66]$.

It is well beyond the scope of this work to compute the RGE equations for the dimension- 8 operators. Instead we content ourselves to make a few comments about the structure of the associated anomalous dimension. The renormalization of a dimension- 8 operator can happen due to one insertion of a dimension- 8 operator in a loop amplitude, two insertions of dimension- 6 operators, or one insertion of dimension- 5 operator and one insertion of a dimension-7 operator. The lattermost type of amplitude will typically be suppressed as was the case with the two dimension- 5 operator contribution to the dimension6 RGE. However, the two dimension- 6 operator contribution to the dimension- 8 RGE will generally be comparable in magnitude to the one dimension- 8 operator contribution. Dimension- 8 is the lowest mass dimension where there are two co-leading contributions to the RGE.

Many of the entries in the anomalous dimension matrix vanish [67] including beyond one-loop [68]. One way to understand these zeroes in the anomalous dimension matrix is through non-renormalization theorem of ref. [67], which applies to loop amplitudes with one dimension- $d$ SMEFT insertion and one SM tree level amplitude. To start define holomorphic and anti-holomorphic weights as $w=n-h$ and $\bar{w}=n+h$, respectively, where $n$ is the number of particles created by an operator and $h$ is the sum of the helicities of the particles created. Then the theorem can be stated as operators belonging to subclass $j$ can renormalize operators of another subclass $i$ if $w_{i} \geq w_{j}$ and $\bar{w}_{i} \geq \bar{w}_{j}$. The theorem can only be violated when the SM tree amplitude contains two Yukawa interactions. The results of the non-renormalization theorem applied to dimension- 8 operators are visualized in table. 19. Visually the theorem transitions down or to the left in the weight lattice.

A contact amplitude resulting from an insertion of dimension- $d$ SMEFT operators obeys the relation

$$
2 d \geq w_{k}+\bar{w}_{k} \geq d .
$$


Furthermore, restricting to particles of spin-1 or less we also have

$$
d \geq\left|w_{k}-\bar{w}_{k}\right|
$$

The relations (6.2) and (6.3) do not, in general, respect the weight bounds of SM tree level amplitudes, $w_{k} \geq 4$ and $\bar{w}_{k} \geq 4$ (which apply to non-exceptional amplitudes). Therefore the theorem needs to be modified to determine vanishing entries among the two dimension- 6 contributions to the dimension- 8 anomalous dimension matrix as the theorem, as currently formulated, relies on the SM tree level weight bounds. It would be interesting to examine in detail the structure of the anomalous dimension matrix resulting from two insertion of dimension- 6 operators.

Inverting the logic of the non-renormalization theorem, operators of the same type will generically mix under RGE evolution. An interesting restriction on how general the mixing comes from Lagrangian terms with multiple flavor structures. Examples of such terms include $Q_{l^{4} H^{2}}, Q_{q^{4} H^{2}}^{(1,3)}, Q_{u^{4} H^{2}}$ and $Q_{d^{4} H^{2}}$. The different flavor structures in these terms can only be mixed by Yukawa contributions to the RGE, i.e. gauge and $\lambda$ contributions do not mix different flavor structures of a given term. See ref. [6] for further discussion on this point. On the other hand, at the dimension-6, there are plenty of example of mixing amongst different terms of the same type. For example, the gauge contribution to the dimension-6 RGE mixes the bosonic operators $C_{H \square}$ and $C_{H D}$ [5]. Along these lines we expect the mixing of $Q_{q^{2} H^{4} D}^{(2)}$ to be closely related to that of $Q_{q^{2} H^{4} D}^{(3)}$ because, as shown in section 3 , these terms contain orthogonal linear combinations of operators. A similar relation is expected for $Q_{l^{2} H^{4} D}^{(2,3)}$.

\section{Conclusions}

In this work we presented a complete basis of dimension- 8 operators in the Standard Model Effective Field Theory. There are 1031 Lagrangian terms, 38 of which vanish in the absence of flavor structure. Multiple checks have been passed including that no operator in the basis can be removed completely using the equations of motion. We also presented a counting based argument that 1031 is the minimum number of Lagrangian terms needed to represent all of the dimension-8 SMEFT operators.

As a sample of what can be done with the a complete basis of dimension- 8 operators, we briefly considered the phenomenology of light-by-light scattering, electroweak precision data, and commented on the structure of the dimension-8 RGE. Additionally, we matched theories of $\mathrm{SU}(2)_{w}$ quartets onto the SMEFT up to dimension- 8 allowing to us showcase the interplay between dimension- 6 effects and dimension- 8 effects, the latter of which cannot be neglected in those models.

Note added. Ref. [69] is set to appear on the arXiv simultaneously with this work. It also presents a complete basis of dimension-8 SMEFT operators. The basis of ref. [69] contains more Lagrangian terms than our basis as it limits its terms to have only one flavor representation, whereas we follow the convention of ref. [2], combining flavor representations together when unambiguously possible to obtain the minimum number of terms. 


\begin{tabular}{|c|c|c|c|c|c|c|}
\hline \multirow{2}{*}{\multicolumn{2}{|c|}{$\bar{w} \frac{}{6}$}} & $X_{L}^{4}$ & $\begin{array}{l}X_{L}^{3} H^{2} \\
X_{L}^{2} \psi^{2} H \\
X_{L} \psi^{4}\end{array}$ & $\begin{array}{l}X_{L}^{2} H^{4} \\
X_{L} \psi^{2} \phi^{3} \\
\psi^{4} H^{2}\end{array}$ & $\psi^{2} H^{5}$ & $H^{8}$ \\
\hline & & & $\begin{array}{l}X_{L}^{2} H^{2} D^{2} \\
X_{L}^{2} \psi \bar{\psi} D \\
X_{L} \psi^{2} H D^{2} \\
\psi^{4} D^{2}\end{array}$ & $\begin{array}{l}X_{L} H^{4} D^{2} \\
X_{L}^{2} \bar{\psi}^{2} H \\
X_{L} \psi \bar{\psi} H^{2} D \\
\psi^{2} H^{3} D^{2} \\
X_{L} \psi^{2} \bar{\psi}^{2} \\
\psi^{3} \bar{\psi} H D\end{array}$ & $\begin{array}{l}H^{6} D^{2} \\
\psi \bar{\psi} H^{4} D \\
\psi^{2} \bar{\psi}^{2} H^{2}\end{array}$ & $\bar{\psi}^{2} H^{5}$ \\
\hline & 4 & & & $\begin{array}{l}X_{L}^{2} X_{R}^{2}, \\
X_{L} X_{R} H^{2} D^{2}, \\
H^{4} D^{4} \\
X_{L} X_{R} \psi \bar{\psi} D \\
X_{R} \psi^{2} H D^{2} \\
X_{L} \bar{\psi}^{2} H D^{2} \\
\psi \bar{\psi} H^{2} D^{3} \\
\psi^{2} \bar{\psi}^{2} D^{2}\end{array}$ & $\begin{array}{l}X_{R} H^{4} D^{2} \\
X_{R}^{2} \psi^{2} H \\
X_{R} \psi \bar{\psi} H^{2} D \\
\bar{\psi}^{2} H^{3} D^{2} \\
X_{R} \psi^{2} \bar{\psi}^{2} \\
\psi \bar{\psi}^{3} H D\end{array}$ & $\begin{array}{l}X_{R}^{2} H^{4}, \\
X_{R} \bar{\psi}^{2} H^{3}, \\
\bar{\psi}^{4} H^{2}\end{array}$ \\
\hline & 2 & & & & $\begin{array}{l}X_{R}^{2} H^{2} D^{2}, \\
X_{R}^{2} \psi \bar{\psi} D, \\
X_{R} \bar{\psi}^{2} H D^{2}, \\
\bar{\psi}^{4} D^{2}\end{array}$ & $\begin{array}{l}X_{R}^{3} H^{2} \\
X_{R}^{2} \bar{\psi}^{2} H, \\
X_{R} \bar{\psi}^{4}\end{array}$ \\
\hline & 0 & & & & & $X_{R}^{4}$ \\
\hline & c & 0 & 2 & 4 & 6 & 8 \\
\hline & & & & $w$ & & \\
\hline
\end{tabular}

Table 19. Weight lattice for dimension- 8 operators in the SMEFT. By the non-renormalization theorem of ref. [67] a subclass of operators, $j$, can renormalize another subclass, $i$, at one-loop if $w_{i} \geq w_{j}$ and $\bar{w}_{i} \geq \bar{w}_{j}$. Visually this prevents transitions down or to the left.

\section{Acknowledgments}

We thank Renato Fonseca, Adam Martin, Grant Remmen, Nicholas Rodd, Verónica Sanz, Michael Trott, the anonymous referee, and especially Jing Shu and Jiang-Hao Yu for useful discussions. The author is grateful for the privilege of being able to stay home and work on a project like this.

\section{A Dimension-6 and -7 operators}

For the sake of convenience we reproduce the tables of dimension- 6 and -7 operators here. Table 20 contains the dimension-7 operators, and is adapted from ref. [17]. The classification scheme we use for the dimension- 7 comes from labelling the classes in table 2 of ref. [10] in descending order. Table 21 contains the dimension- 6 operators, and is adapted 


\begin{tabular}{|c|c|}
\hline \multicolumn{2}{|c|}{$\mathbf{1}: \boldsymbol{\psi}^{\mathbf{2}} \boldsymbol{X} \boldsymbol{H}^{\mathbf{2}}+$ h.c. } \\
\hline$Q_{l^{2} W H^{2}}$ & $\epsilon_{m n}\left(\tau^{I} \epsilon\right)_{j k}\left(l_{p}^{m} C \sigma^{\mu \nu} l_{r}^{j}\right) H^{n} H^{k} W_{\mu \nu}^{I}$ \\
\hline$Q_{l^{2} B H^{2}}$ & $\epsilon_{m n} \epsilon_{j k}\left(l_{p}^{m} C \sigma^{\mu \nu} l_{r}^{j}\right) H^{n} H^{k} B_{\mu \nu}$ \\
\hline
\end{tabular}

\begin{tabular}{|l|l|}
\hline \multicolumn{2}{|c|}{$\mathbf{2}: \boldsymbol{\psi}^{\mathbf{2}} \boldsymbol{H}^{\mathbf{4}}+$ h.c. } \\
\hline$Q_{l^{2} H^{4}}$ & $\epsilon_{m n} \epsilon_{j k}\left(l_{p}^{m} C l_{r}^{j}\right) H^{n} H^{k}\left(H^{\dagger} H\right)$ \\
\hline
\end{tabular}

\begin{tabular}{|l|c|}
\hline \multicolumn{2}{|c|}{$\mathbf{3}(\boldsymbol{B}): \boldsymbol{\psi}^{\mathbf{4}} \boldsymbol{H}+$ h.c. } \\
\hline$Q_{l^{3} e H}$ & $\epsilon_{j k} \epsilon_{m n}\left(\bar{e}_{p} l_{r}^{j}\right)\left(l_{s}^{k} C l_{t}^{m}\right) H^{n}$ \\
$Q_{l e u d H}$ & $\epsilon_{j k}\left(\bar{d}_{p} l_{r}^{j}\right)\left(u_{s} C e_{t}\right) H^{k}$ \\
$Q_{l^{2} q d H}^{(1)}$ & $\epsilon_{j k} \epsilon_{m n}\left(\bar{d}_{p} l_{r}^{j}\right)\left(q_{s}^{k} C l_{t}^{m}\right) H^{n}$ \\
$Q_{l^{2} q d H}^{(2)}$ & $\epsilon_{j m} \epsilon_{k n}\left(\bar{d}_{p} l_{r}^{j}\right)\left(q_{s}^{k} C l_{t}^{m}\right) H^{n}$ \\
$Q_{l^{2} q u H}$ & $\epsilon_{j k}\left(\bar{q}_{p}^{m} u_{r}\right)\left(l_{s m} C l_{t}^{j}\right) H^{k}$ \\
\hline
\end{tabular}

\begin{tabular}{|c|c|}
\hline \multicolumn{2}{|c|}{$\mathbf{3}(\not \boldsymbol{B}): \boldsymbol{\psi}^{\mathbf{4}} \boldsymbol{H}+$ h.c. } \\
\hline$Q_{l u d^{2} H}$ & $\epsilon_{\alpha \beta \gamma}\left(\bar{l}_{p} d_{r}^{\alpha}\right)\left(u_{s}^{\beta} C d_{t}^{\gamma}\right) \widetilde{H}$ \\
$Q_{l q^{2} d H}$ & $\epsilon_{\alpha \beta \gamma}\left(\bar{l}_{p}^{m} d_{r}^{\alpha}\right)\left(q_{s m}^{\beta} C q_{t}^{j \gamma}\right) H_{j}^{\dagger}$ \\
\hdashline$Q_{l d^{3} H}$ & $\epsilon_{\alpha \beta \gamma}\left(\bar{l}_{p} d_{r}^{\alpha}\right)\left(d_{s}^{\beta} C d_{t}^{\gamma}\right) H$ \\
$Q_{e q d^{2} H}$ & $\epsilon_{\alpha \beta \gamma}\left(\bar{e}_{p} q_{r}^{j \alpha}\right)\left(d_{s}^{\beta} C d_{t}^{\gamma}\right) H_{j}^{\dagger}$ \\
\hline
\end{tabular}

\begin{tabular}{|l|l|}
\hline \multicolumn{2}{|c|}{$\mathbf{4}: \boldsymbol{\psi}^{\mathbf{2}} \boldsymbol{H}^{\mathbf{3}} \boldsymbol{D}+$ h.c. } \\
\hline$Q_{l e H^{3} D}$ & $\epsilon_{m n} \epsilon_{j k}\left(l_{p}^{m} C \gamma^{\mu} e_{r}\right) H^{n} H^{j} i D_{\mu} H^{k}$ \\
\hline
\end{tabular}

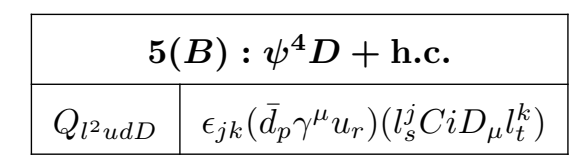

\begin{tabular}{|l|c|}
\hline \multicolumn{2}{|c|}{$\mathbf{6}: \boldsymbol{\psi}^{\mathbf{2}} \boldsymbol{H}^{\mathbf{2}} \boldsymbol{D}^{\mathbf{2}}+$ h.c. } \\
\hline$Q_{l^{2} H^{2} D^{2}}^{(1)}$ & $\epsilon_{j k} \epsilon_{m n}\left(l_{p}^{j} C D^{\mu} l_{r}^{k}\right) H^{m}\left(D_{\mu} H^{n}\right)$ \\
$Q_{l^{2} H^{2} D^{2}}^{(2)}$ & $\epsilon_{j m} \epsilon_{k n}\left(l_{p}^{j} C D^{\mu} l_{r}^{k}\right) H^{m}\left(D_{\mu} H^{n}\right)$ \\
\hline
\end{tabular}

\begin{tabular}{|c|c|}
\hline \multicolumn{2}{|c|}{$\mathbf{5}(\not \boldsymbol{B}): \boldsymbol{\psi}^{\mathbf{4}} \boldsymbol{D}+$ h.c. } \\
\hline$Q_{l q d^{2} D}$ & $\epsilon_{\alpha \beta \gamma}\left(\bar{l}_{p} \gamma^{\mu} q_{r}^{\alpha}\right)\left(d_{s}^{\beta} C i D_{\mu} d_{t}^{\gamma}\right)$ \\
$Q_{e d^{3} D}$ & $\epsilon_{\alpha \beta \gamma}\left(\bar{e}_{p} \gamma^{\mu} d_{r}^{\alpha}\right)\left(d_{s}^{\beta} C i D_{\mu} d_{t}^{\gamma}\right)$ \\
\hline
\end{tabular}

Table 20. The dimension-seven operators in the SMEFT. The operators are divided into six classes according to their field content. The classes- 3 and -5 are further divided into subclasses according to their baryon number. All of the operators have Hermitian conjugates. The subscripts $p, r, s, t$ are weak-eigenstate indices. Operators below the dashed lines in classes- 1 and -3 vanish when there is only one generation of fermions.

from ref. [5]. In contrast with [5] we also include in table 21 the baryon number violating operators as listed in ref. [6]. 


\begin{tabular}{|c|c|}
\hline \multicolumn{2}{|c|}{$1: \boldsymbol{X}^{\mathbf{3}}$} \\
\hline$Q_{G}$ & $f^{A B C} G_{\mu}^{A \nu} G_{\nu}^{B \rho} G_{\rho}^{C \mu}$ \\
$Q_{\widetilde{G}}$ & $f^{A B C} \widetilde{G}_{\mu}^{A \nu} G_{\nu}^{B \rho} G_{\rho}^{C \mu}$ \\
$Q_{W}$ & $\epsilon^{I J K} W_{\mu}^{I \nu} W_{\nu}^{J \rho} W_{\rho}^{K \mu}$ \\
$Q_{\widetilde{W}}$ & $\epsilon^{I J K} \widetilde{W}_{\mu}^{I \nu} W_{\nu}^{J \rho} W_{\rho}^{K \mu}$ \\
\hline
\end{tabular}

\begin{tabular}{|c|c|c|c|c|c|}
\hline \multicolumn{2}{|c|}{$2: H^{6}$} & \multicolumn{2}{|r|}{$3: H^{4} D^{2}$} & \multicolumn{2}{|c|}{$5: \psi^{2} H^{3}+$ h.c. } \\
\hline$Q_{H}$ & $\left(H^{\dagger} H\right)^{3}$ & $Q_{H \square}$ & $\left(H^{\dagger} H\right) \square\left(H^{\dagger} H\right)$ & $Q_{e H}$ & $\left(H^{\dagger} H\right)\left(\bar{l}_{p} e_{r} H\right)$ \\
\hline & & $Q_{H D}$ & $\left(H^{\dagger} D^{\mu} H\right)^{*}\left(H^{\dagger} D_{\mu} H\right)$ & $Q_{u H}$ & $\left(H^{\dagger} H\right)\left(\bar{q}_{p} u_{r} \widetilde{H}\right)$ \\
\hline & & & & $Q_{d H}$ & $\left(H^{\dagger} H\right)\left(\bar{q}_{p} d_{r} H\right)$ \\
\hline
\end{tabular}

\begin{tabular}{|c|c|}
\hline \multicolumn{2}{|c|}{$\mathbf{4}: \boldsymbol{X}^{2} \boldsymbol{H}^{\mathbf{2}}$} \\
\hline$Q_{H G}$ & $H^{\dagger} H G_{\mu \nu}^{A} G^{A \mu \nu}$ \\
$Q_{H \widetilde{G}}$ & $H^{\dagger} H \widetilde{G}_{\mu \nu}^{A} G^{A \mu \nu}$ \\
$Q_{H W}$ & $H^{\dagger} H W_{\mu \nu}^{I} W^{I \mu \nu}$ \\
$Q_{H \widetilde{W}}$ & $H^{\dagger} H \widetilde{W}_{\mu \nu}^{I} W^{I \mu \nu}$ \\
$Q_{H B}$ & $H^{\dagger} H B_{\mu \nu} B^{\mu \nu}$ \\
$Q_{H \widetilde{B}}$ & $H^{\dagger} H \widetilde{B}_{\mu \nu} B^{\mu \nu}$ \\
$Q_{H W B}$ & $H^{\dagger} \tau^{I} H W_{\mu \nu}^{I} B^{\mu \nu}$ \\
$Q_{H \widetilde{W} B}$ & $H^{\dagger} \tau^{I} H \widetilde{W}_{\mu \nu}^{I} B^{\mu \nu}$ \\
\hline
\end{tabular}

\begin{tabular}{|c|c|}
\hline \multicolumn{2}{|c|}{$\mathbf{6}: \boldsymbol{\psi}^{2} \boldsymbol{X} \boldsymbol{H}+\mathbf{h . c .}$} \\
\hline$Q_{e W}$ & $\left(\bar{l}_{p} \sigma^{\mu \nu} e_{r}\right) \tau^{I} H W_{\mu \nu}^{I}$ \\
$Q_{e B}$ & $\left(\bar{l}_{p} \sigma^{\mu \nu} e_{r}\right) H B_{\mu \nu}$ \\
$Q_{u G}$ & $\left(\bar{q}_{p} \sigma^{\mu \nu} T^{A} u_{r}\right) \widetilde{H} G_{\mu \nu}^{A}$ \\
$Q_{u W}$ & $\left(\bar{q}_{p} \sigma^{\mu \nu} u_{r}\right) \tau^{I} \widetilde{H} W_{\mu \nu}^{I}$ \\
$Q_{u B}$ & $\left(\bar{q}_{p} \sigma^{\mu \nu} u_{r}\right) \widetilde{H} B_{\mu \nu}$ \\
$Q_{d G}$ & $\left(\bar{q}_{p} \sigma^{\mu \nu} T^{A} d_{r}\right) H G_{\mu \nu}^{A}$ \\
$Q_{d W}$ & $\left(\bar{q}_{p} \sigma^{\mu \nu} d_{r}\right) \tau^{I} H W_{\mu \nu}^{I}$ \\
$Q_{d B}$ & $\left(\bar{q}_{p} \sigma^{\mu \nu} d_{r}\right) H B_{\mu \nu}$ \\
\hline
\end{tabular}

\begin{tabular}{|c|c|}
\hline \multicolumn{2}{|c|}{$\mathbf{7}: \boldsymbol{\psi}^{2} \boldsymbol{H}^{2} \boldsymbol{D}$} \\
\hline$Q_{H l}^{(1)}$ & $\left(H^{\dagger} i \overleftrightarrow{D}_{\mu} H\right)\left(\bar{l}_{p} \gamma^{\mu} l_{r}\right)$ \\
$Q_{H l}^{(3)}$ & $\left(H^{\dagger} i \overleftrightarrow{D}_{\mu}^{I} H\right)\left(\bar{l}_{p} \tau^{I} \gamma^{\mu} l_{r}\right)$ \\
$Q_{H e}$ & $\left(H^{\dagger} i \overleftrightarrow{D}_{\mu} H\right)\left(\bar{e}_{p} \gamma^{\mu} e_{r}\right)$ \\
$Q_{H q}^{(1)}$ & $\left(H^{\dagger} i \overleftrightarrow{D}_{\mu} H\right)\left(\bar{q}_{p} \gamma^{\mu} q_{r}\right)$ \\
$Q_{H q}^{(3)}$ & $\left(H^{\dagger} i \overleftrightarrow{D}_{\mu}^{I} H\right)\left(\bar{q}_{p} \tau^{I} \gamma^{\mu} q_{r}\right)$ \\
$Q_{H u}$ & $\left(H^{\dagger} i \overleftrightarrow{D}_{\mu} H\right)\left(\bar{u}_{p} \gamma^{\mu} u_{r}\right)$ \\
$Q_{H d}$ & $\left(H^{\dagger} i \overleftrightarrow{D}_{\mu} H\right)\left(\bar{d}_{p} \gamma^{\mu} d_{r}\right)$ \\
$Q_{H u d}+$ h.c. & $i\left(\widetilde{H}^{\dagger} D_{\mu} H\right)\left(\bar{u}_{p} \gamma^{\mu} d_{r}\right)$ \\
\hline
\end{tabular}

\begin{tabular}{|c|c|}
\hline \multicolumn{2}{|c|}{$\mathbf{8}:(\overline{\boldsymbol{L}} \boldsymbol{L})(\overline{\boldsymbol{L}} \boldsymbol{L})$} \\
\hline$Q_{l l}$ & $\left(\bar{l}_{p} \gamma^{\mu} l_{r}\right)\left(\bar{l}_{s} \gamma_{\mu} l_{t}\right)$ \\
$Q_{q q}^{(1)}$ & $\left(\bar{q}_{p} \gamma^{\mu} q_{r}\right)\left(\bar{q}_{s} \gamma_{\mu} q_{t}\right)$ \\
$Q_{q q}^{(3)}$ & $\left(\bar{q}_{p} \gamma^{\mu} \tau^{I} q_{r}\right)\left(\bar{q}_{s} \gamma_{\mu} \tau^{I} q_{t}\right)$ \\
$Q_{l q}^{(1)}$ & $\left(\bar{l}_{p} \gamma^{\mu} l_{r}\right)\left(\bar{q}_{s} \gamma_{\mu} q_{t}\right)$ \\
$Q_{l q}^{(3)}$ & $\left(\bar{l}_{p} \gamma^{\mu} \tau^{I} l_{r}\right)\left(\bar{q}_{s} \gamma_{\mu} \tau^{I} q_{t}\right)$ \\
\hline
\end{tabular}

\begin{tabular}{|c|c|}
\hline \multicolumn{2}{|c|}{$\mathbf{8}:(\overline{\boldsymbol{R}} \boldsymbol{R})(\overline{\boldsymbol{R}} \boldsymbol{R})$} \\
\hline$Q_{e e}$ & $\left(\bar{e}_{p} \gamma^{\mu} e_{r}\right)\left(\bar{e}_{s} \gamma_{\mu} e_{t}\right)$ \\
$Q_{u u}$ & $\left(\bar{u}_{p} \gamma^{\mu} u_{r}\right)\left(\bar{u}_{s} \gamma_{\mu} u_{t}\right)$ \\
$Q_{d d}$ & $\left(\bar{d}_{p} \gamma^{\mu} d_{r}\right)\left(\bar{d}_{s} \gamma_{\mu} d_{t}\right)$ \\
$Q_{e u}$ & $\left(\bar{e}_{p} \gamma^{\mu} e_{r}\right)\left(\bar{u}_{s} \gamma_{\mu} u_{t}\right)$ \\
$Q_{e d}$ & $\left(\bar{e}_{p} \gamma^{\mu} e_{r}\right)\left(\bar{d}_{s} \gamma_{\mu} d_{t}\right)$ \\
$Q_{u d}^{(1)}$ & $\left(\bar{u}_{p} \gamma^{\mu} u_{r}\right)\left(\bar{d}_{s} \gamma_{\mu} d_{t}\right)$ \\
$Q_{u d}^{(8)}$ & $\left(\bar{u}_{p} \gamma^{\mu} T^{A} u_{r}\right)\left(\bar{d}_{s} \gamma_{\mu} T^{A} d_{t}\right)$ \\
\hline
\end{tabular}

\begin{tabular}{|c|c|}
\hline \multicolumn{2}{|c|}{$\mathbf{8}:(\overline{\boldsymbol{L}} \boldsymbol{L})(\overline{\boldsymbol{R}} \boldsymbol{R})$} \\
\hline$Q_{l e}$ & $\left(\bar{l}_{p} \gamma^{\mu} l_{r}\right)\left(\bar{e}_{s} \gamma_{\mu} e_{t}\right)$ \\
$Q_{l u}$ & $\left(\bar{l}_{p} \gamma^{\mu} l_{r}\right)\left(\bar{u}_{s} \gamma_{\mu} u_{t}\right)$ \\
$Q_{l d}$ & $\left(\bar{l}_{p} \gamma^{\mu} l_{r}\right)\left(\bar{d}_{s} \gamma_{\mu} d_{t}\right)$ \\
$Q_{q e}$ & $\left(\bar{q}_{p} \gamma^{\mu} q_{r}\right)\left(\bar{e}_{s} \gamma_{\mu} e_{t}\right)$ \\
$Q_{q u}^{(1)}$ & $\left(\bar{q}_{p} \gamma^{\mu} q_{r}\right)\left(\bar{u}_{s} \gamma_{\mu} u_{t}\right)$ \\
$Q_{q u}^{(8)}$ & $\left(\bar{q}_{p} \gamma^{\mu} T^{A} q_{r}\right)\left(\bar{u}_{s} \gamma_{\mu} T^{A} u_{t}\right)$ \\
$Q_{q d}^{(1)}$ & $\left(\bar{q}_{p} \gamma^{\mu} q_{r}\right)\left(\bar{d}_{s} \gamma_{\mu} d_{t}\right)$ \\
$Q_{q d}^{(8)}$ & $\left(\bar{q}_{p} \gamma^{\mu} T^{A} q_{r}\right)\left(\bar{d}_{s} \gamma_{\mu} T^{A} d_{t}\right)$ \\
\hline
\end{tabular}

\begin{tabular}{|l|c|}
\hline \multicolumn{2}{|c|}{$\mathbf{8}:(\overline{\boldsymbol{L}} \boldsymbol{R})(\overline{\boldsymbol{R}} \boldsymbol{L})+$ h.c. } \\
\hline$Q_{l e d q}$ & $\left(\bar{l}_{p}^{j} e_{r}\right)\left(\bar{d}_{s} q_{t j}\right)$ \\
\hline
\end{tabular}

\begin{tabular}{|l|c|}
\hline \multicolumn{2}{|c|}{$\mathbf{8}:(\overline{\boldsymbol{L}} \boldsymbol{R})(\overline{\boldsymbol{L}} \boldsymbol{R})+$ h.c. } \\
\hline$Q_{q u q d}^{(1)}$ & $\left(\bar{q}_{p}^{j} u_{r}\right) \epsilon_{j k}\left(\bar{q}_{s}^{k} d_{t}\right)$ \\
$Q_{q u q d}^{(8)}$ & $\left(\bar{q}_{p}^{j} T^{A} u_{r}\right) \epsilon_{j k}\left(\bar{q}_{s}^{k} T^{A} d_{t}\right)$ \\
$Q_{\text {lequ }}^{(1)}$ & $\left(\bar{l}_{p}^{j} e_{r}\right) \epsilon_{j k}\left(\bar{q}_{s}^{k} u_{t}\right)$ \\
$Q_{\text {lequ }}^{(3)}$ & $\left(\bar{l}_{p}^{j} \sigma_{\mu \nu} e_{r}\right) \epsilon_{j k}\left(\bar{q}_{s}^{k} \sigma^{\mu \nu} u_{t}\right)$ \\
\hline
\end{tabular}

\begin{tabular}{|c|c|}
\hline \multicolumn{2}{|c|}{$\mathbf{8}:(\not \boldsymbol{B})+$ h.c. } \\
\hline$Q_{d u q l}$ & $\epsilon_{\alpha \beta \gamma} \epsilon_{j k}\left(d_{p}^{\alpha} C u_{r}^{\beta}\right)\left(q_{s}^{j \gamma} C l_{t}^{k}\right)$ \\
$Q_{q q u e}$ & $\epsilon_{\alpha \beta \gamma} \epsilon_{j k}\left(q_{p}^{j \alpha} C q_{r}^{k \beta}\right)\left(u_{s}^{\gamma} C e_{t}\right)$ \\
$Q_{q q q l}$ & $\epsilon_{\alpha \beta \gamma} \epsilon_{m n} \epsilon_{j k}\left(q_{p}^{m \alpha} C q_{r}^{j \beta}\right)\left(q_{s}^{k \gamma} C l_{t}^{n}\right)$ \\
$Q_{\text {duue }}$ & $\epsilon_{\alpha \beta \gamma}\left(d_{p}^{\alpha} C u_{r}^{\beta}\right)\left(u_{s}^{\gamma} C e_{t}\right)$ \\
\hline
\end{tabular}

Table 21. The dimension-six operators in the SMEFT. The operators are divided into eight classes according to their field content. The class- $8 \psi^{4}$ four-fermion operators are further divided into subclasses according to their chiral and baryonic properties. Operators with + h.c. have Hermitian conjugates, as does the $\psi^{2} H^{2} D$ operator $Q_{H u d}$. The subscripts $p, r, s, t$ are weak-eigenstate indices. 
Open Access. This article is distributed under the terms of the Creative Commons Attribution License (CC-BY 4.0), which permits any use, distribution and reproduction in any medium, provided the original author(s) and source are credited.

\section{References}

[1] S. Weinberg, Baryon and lepton nonconserving processes, Phys. Rev. Lett. 43 (1979) 1566 [INSPIRE].

[2] B. Grzadkowski, M. Iskrzynski, M. Misiak and J. Rosiek, Dimension-six terms in the Standard Model Lagrangian, JHEP 10 (2010) 085 [arXiv: 1008.4884] [INSPIRE].

[3] W. Buchmüller and D. Wyler, Effective Lagrangian analysis of new interactions and flavor conservation, Nucl. Phys. B 268 (1986) 621 [INSPIRE].

[4] L.F. Abbott and M.B. Wise, The effective Hamiltonian for nucleon decay, Phys. Rev. D 22 (1980) 2208 [INSPIRE].

[5] R. Alonso, E.E. Jenkins, A.V. Manohar and M. Trott, Renormalization group evolution of the Standard Model dimension six operators III: gauge coupling dependence and phenomenology, JHEP 04 (2014) 159 [arXiv:1312.2014] [INSPIRE].

[6] R. Alonso, H.-M. Chang, E.E. Jenkins, A.V. Manohar and B. Shotwell, Renormalization group evolution of dimension-six baryon number violating operators, Phys. Lett. B 734 (2014) 302 [arXiv: 1405.0486] [INSPIRE].

[7] L. Lehman and A. Martin, Hilbert series for constructing Lagrangians: expanding the phenomenologist's toolbox, Phys. Rev. D 91 (2015) 105014 [arXiv: 1503.07537] [InSPIRE].

[8] B. Henning, X. Lu, T. Melia and H. Murayama, Hilbert series and operator bases with derivatives in Effective Field Theories, Commun. Math. Phys. 347 (2016) 363 [arXiv: 1507.07240] [INSPIRE].

[9] L. Lehman and A. Martin, Low-derivative operators of the Standard Model Effective Field Theory via Hilbert series methods, JHEP 02 (2016) 081 [arXiv: 1510.00372] [INSPIRE].

[10] B. Henning, X. Lu, T. Melia and H. Murayama, 2, 84, 30, 993, 560, 15456, 11962, 261485, ...: higher dimension operators in the SM EFT, JHEP 08 (2017) 016 [Erratum ibid. 09 (2019) 019] [arXiv: 1512.03433] [INSPIRE].

[11] R.M. Fonseca, The Sym2Int program: going from symmetries to interactions, J. Phys. Conf. Ser. 873 (2017) 012045 [arXiv: 1703.05221] [INSPIRE].

[12] B. Gripaios and D. Sutherland, DEFT: a program for operators in EFT, JHEP 01 (2019) 128 [arXiv: 1807.07546] [INSPIRE].

[13] J.C. Criado, BasisGen: automatic generation of operator bases, Eur. Phys. J. C 79 (2019) 256 [arXiv: 1901.03501] [INSPIRE].

[14] C.B. Marinissen, R. Rahn and W.J. Waalewijn, ..., 83106786, 114382724, 1509048322, 2343463290, 27410087742, .. efficient Hilbert series for effective theories, Phys. Lett. B 808 (2020) 135632 [arXiv:2004.09521] [INSPIRE].

[15] U. Banerjee, J. Chakrabortty, S. Prakash and S.U. Rahaman, Characters and group invariant polynomials of (super)fields: road to "Lagrangian", Eur. Phys. J. C 80 (2020) 938 [arXiv: 2004.12830] [INSPIRE]. 
[16] L. Lehman, Extending the Standard Model Effective Field Theory with the complete set of dimension-7 operators, Phys. Rev. D 90 (2014) 125023 [arXiv:1410.4193] [INSPIRE].

[17] Y. Liao and X.-D. Ma, Renormalization group evolution of dimension-seven baryon- and lepton-number-violating operators, JHEP 11 (2016) 043 [arXiv: 1607.07309] [INSPIRE].

[18] A. Morozov, Matrix of mixing of scalar and vector mesons of dimension $D \leq 8$ in $Q C D$ (in Russian), Sov. J. Nucl. Phys. 40 (1984) 505 [Yad. Fiz. 40 (1984) 788] [inSPIRE].

[19] C. Hays, A. Martin, V. Sanz and J. Setford, On the impact of dimension-eight SMEFT operators on Higgs measurements, JHEP 02 (2019) 123 [arXiv: 1808.00442] [INSPIRE].

[20] G.N. Remmen and N.L. Rodd, Consistency of the Standard Model Effective Field Theory, JHEP 12 (2019) 032 [arXiv: 1908.09845] [INSPIRE].

[21] B. Grinstein and M.B. Wise, Operator analysis for precision electroweak physics, Phys. Lett. B 265 (1991) 326 [INSPIRE].

[22] S. Dawson and C.W. Murphy, Standard Model EFT and extended scalar sectors, Phys. Rev. D 96 (2017) 015041 [arXiv:1704.07851] [INSPIRE].

[23] R.M. Fonseca, Enumerating the operators of an Effective Field Theory, Phys. Rev. D 101 (2020) 035040 [arXiv: 1907.12584] [INSPIRE].

[24] S. Alioli, R. Boughezal, E. Mereghetti and F. Petriello, Novel angular dependence in Drell-Yan lepton production via dimension-8 operators, Phys. Lett. B 809 (2020) 135703 [arXiv: 2003.11615] [INSPIRE].

[25] G.N. Remmen and N.L. Rodd, Flavor constraints from unitarity and analyticity, Phys. Rev. Lett. 125 (2020) 081601 [arXiv: 2004.02885] [INSPIRE].

[26] E.E. Jenkins, A.V. Manohar and M. Trott, Renormalization group evolution of the Standard Model dimension six operators I: formalism and $\lambda$ dependence, JHEP 10 (2013) 087 [arXiv: 1308.2627] [INSPIRE].

[27] R.F. Dashen, E.E. Jenkins and A.V. Manohar, Spin flavor structure of large $N_{c}$ baryons, Phys. Rev. D 51 (1995) 3697 [hep-ph/9411234] [InSPIRE].

[28] O. Halpern, Scattering processes produced by electrons in negative energy states, Phys. Rev. 44 (1933) 855.2 [INSPIRE].

[29] H. Euler and B. Kockel, The scattering of light by light in Dirac's theory, Naturwiss. 23 (1935) 246 [INSPIRE].

[30] W. Heisenberg and H. Euler, Consequences of Dirac's theory of positrons, Z. Phys. 98 (1936) 714 [physics/0605038] [INSPIRE].

[31] H. Euler, On the scattering of light by light according to Dirac's theory, Annalen Phys. 26 (1936) 398 [INSPIRE].

[32] R. Karplus and M. Neuman, Non-linear interactions between electromagnetic fields, Phys. Rev. 80 (1950) 380 [INSPIRE].

[33] R. Karplus and M. Neuman, The scattering of light by light, Phys. Rev. 83 (1951) 776 [INSPIRE].

[34] ATLAS collaboration, Observation of light-by-light scattering in ultraperipheral $\mathrm{Pb}+\mathrm{Pb}$ collisions with the ATLAS detector, Phys. Rev. Lett. 123 (2019) 052001 [arXiv:1904.03536] [INSPIRE]. 
[35] CMS collaboration, Evidence for light-by-light scattering and searches for axion-like particles in ultraperipheral $\mathrm{Pb}-\mathrm{Pb}$ collisions at $\sqrt{s_{\mathrm{NN}}}=5.02 \mathrm{TeV}$, Phys. Lett. B 797 (2019) 134826 [arXiv: 1810.04602] [INSPIRE].

[36] A.V. Manohar and V. Mateu, Dispersion relation bounds for $\pi \pi$ scattering, Phys. Rev. D 77 (2008) 094019 [arXiv:0801.3222] [INSPIRE].

[37] B. Bellazzini, Softness and amplitudes' positivity for spinning particles, JHEP 02 (2017) 034 [arXiv: 1605.06111] [INSPIRE].

[38] J.F. Gunion, H.E. Haber and J. Wudka, Sum rules for Higgs bosons, Phys. Rev. D 43 (1991) 904 [INSPIRE].

[39] B. Grinstein, C.W. Murphy, D. Pirtskhalava and P. Uttayarat, Theoretical constraints on additional Higgs bosons in light of the 126 GeV Higgs, JHEP 05 (2014) 083 [arXiv: 1401.0070] [INSPIRE].

[40] B. Bellazzini and F. Riva, New phenomenological and theoretical perspective on anomalous $Z Z$ and $Z \gamma$ processes, Phys. Rev. D 98 (2018) 095021 [arXiv: 1806.09640] [InSPIRE].

[41] C. Zhang and S.-Y. Zhou, Positivity bounds on vector boson scattering at the LHC, Phys. Rev. D 100 (2019) 095003 [arXiv: 1808.00010] [INSPIRE].

[42] Q. Bi, C. Zhang and S.-Y. Zhou, Positivity constraints on aQGC: carving out the physical parameter space, JHEP 06 (2019) 137 [arXiv: 1902.08977] [INSPIRE].

[43] M. Froissart, Asymptotic behavior and subtractions in the Mandelstam representation, Phys. Rev. 123 (1961) 1053 [INSPIRE].

[44] J.S. Schwinger, On gauge invariance and vacuum polarization, Phys. Rev. 82 (1951) 664 [INSPIRE].

[45] M.E. Peskin and T. Takeuchi, A new constraint on a strongly interacting Higgs sector, Phys. Rev. Lett. 65 (1990) 964 [inSPIRE].

[46] W.J. Marciano and J.L. Rosner, Atomic parity violation as a probe of new physics, Phys. Rev. Lett. 65 (1990) 2963 [Erratum ibid. 68 (1992) 898] [INSPIRE].

[47] D.C. Kennedy and P. Langacker, Precision electroweak experiments and heavy physics: a global analysis, Phys. Rev. Lett. 65 (1990) 2967 [Erratum ibid. 66 (1991) 395] [INSPIRE].

[48] B. Holdom and J. Terning, Large corrections to electroweak parameters in technicolor theories, Phys. Lett. B 247 (1990) 88 [InSPIRE].

[49] M. Golden and L. Randall, Radiative corrections to electroweak parameters in technicolor theories, Nucl. Phys. B 361 (1991) 3 [InSPIRE].

[50] G. Altarelli and R. Barbieri, Vacuum polarization effects of new physics on electroweak processes, Phys. Lett. B 253 (1991) 161 [INSPIRE].

[51] J. Ellis, C.W. Murphy, V. Sanz and T. You, Updated global SMEFT fit to Higgs, diboson and electroweak data, JHEP 06 (2018) 146 [arXiv:1803.03252] [INSPIRE].

[52] A. Helset, M. Paraskevas and M. Trott, Gauge fixing the Standard Model Effective Field Theory, Phys. Rev. Lett. 120 (2018) 251801 [arXiv:1803.08001] [InSPIRE].

[53] A. Helset, A. Martin and M. Trott, The geometric Standard Model Effective Field Theory, JHEP 03 (2020) 163 [arXiv:2001.01453] [INSPIRE]. 
[54] C. Hays, A. Helset, A. Martin and M. Trott, Exact SMEFT formulation and expansion to $O\left(v^{4} / \Lambda^{4}\right)$, arXiv: 2007.00565 [INSPIRE].

[55] CMS collaboration, Combination of searches for Higgs boson pair production in proton-proton collisions at $\sqrt{s}=13$ TeV, Phys. Rev. Lett. 122 (2019) 121803 [arXiv: 1811.09689] [INSPIRE].

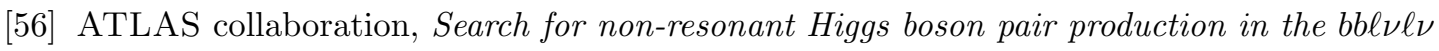
final state with the ATLAS detector in pp collisions at $\sqrt{s}=13$ TeV, Phys. Lett. B 801 (2020) 135145 [arXiv: 1908.06765] [INSPIRE].

[57] ALEPH, DELPHI, L3, OPAL, SLD, LEP Electroweak Working Group, SLD Electroweak Group and SLD Heavy Flavour Group collaborations, Precision electroweak measurements on the $Z$ resonance, Phys. Rept. 427 (2006) 257 [hep-ex/0509008] [INSPIRE].

[58] B. Henning, X. Lu and H. Murayama, How to use the Standard Model Effective Field Theory, JHEP 01 (2016) 023 [arXiv: 1412.1837] [INSPIRE].

[59] J. de Blas, J.C. Criado, M. Pérez-Victoria and J. Santiago, Effective description of general extensions of the Standard Model: the complete tree-level dictionary, JHEP 03 (2018) 109 [arXiv: 1711.10391] [INSPIRE].

[60] S. Das Bakshi, J. Chakrabortty and S.K. Patra, CoDEx: Wilson coefficient calculator connecting SMEFT to UV theory, Eur. Phys. J. C 79 (2019) 21 [arXiv:1808.04403] [INSPIRE].

[61] K.S. Babu, C.N. Leung and J.T. Pantaleone, Renormalization of the neutrino mass operator, Phys. Lett. B 319 (1993) 191 [hep-ph/9309223] [INSPIRE].

[62] E.E. Jenkins, A.V. Manohar and M. Trott, Renormalization group evolution of the Standard Model dimension six operators II: Yukawa dependence, JHEP 01 (2014) 035 [arXiv: 1310.4838] [INSPIRE].

[63] Y. Liao and X.-D. Ma, Renormalization group evolution of dimension-seven operators in Standard Model Effective Field Theory and relevant phenomenology, JHEP 03 (2019) 179 [arXiv: 1901.10302] [INSPIRE].

[64] S. Davidson, M. Gorbahn and M. Leak, Majorana neutrino masses in the renormalization group equations for lepton flavor violation, Phys. Rev. D 98 (2018) 095014 [arXiv: 1807.04283] [INSPIRE].

[65] A. Kobach, Baryon number, lepton number, and operator dimension in the Standard Model, Phys. Lett. B 758 (2016) 455 [arXiv: 1604.05726] [INSPIRE].

[66] A. Helset and A. Kobach, Baryon number, lepton number, and operator dimension in the SMEFT with flavor symmetries, Phys. Lett. B 800 (2020) 135132 [arXiv:1909.05853] [INSPIRE].

[67] C. Cheung and C.-H. Shen, Nonrenormalization theorems without supersymmetry, Phys. Rev. Lett. 115 (2015) 071601 [arXiv: 1505.01844] [INSPIRE].

[68] Z. Bern, J. Parra-Martinez and E. Sawyer, Structure of two-loop SMEFT anomalous dimensions via on-shell methods, arXiv:2005.12917 [INSPIRE].

[69] H.-L. Li, Z. Ren, J. Shu, M.-L. Xiao, J.-H. Yu and Y.-H. Zheng, Complete set of dimension-8 operators in the Standard Model Effective Field Theory, arXiv:2005.00008 [InSPIRE]. 